\title{
GEOMETRIC DATA FITTING
}

\author{
JOSÉ L. MARTÍNEZ-MORALES
}

Received 21 April 2003

Given a dense set of points lying on or near an embedded submanifold $M_{0} \subset \mathbb{R}^{n}$ of Euclidean space, the manifold fitting problem is to find an embedding $F: M \rightarrow \mathbb{R}^{n}$ that approximates $M_{0}$ in the sense of least squares. When the dataset is modeled by a probability distribution, the fitting problem reduces to that of finding an embedding that minimizes $E_{d}[F]$, the expected square of the distance from a point in $\mathbb{R}^{n}$ to $F(M)$. It is shown that this approach to the fitting problem is guaranteed to fail because the functional $E_{d}$ has no local minima. This problem is addressed by adding a small multiple $k$ of the harmonic energy functional to the expected square of the distance. Techniques from the calculus of variations are then used to study this modified functional.

\section{Introduction}

In this paper we are concerned with the following problem. Let $M_{0}$ be the image of a smooth embedding $F_{0}: M \rightarrow \mathbb{R}^{n}$, where $M$ is a smooth, compact manifold without boundary, and let $\mathbf{Y}=\left\{\mathbf{y}_{1}, \ldots, \mathbf{y}_{q}\right\} \subset \mathbb{R}^{n}$ be a collection of points that we assume to be contained in a smooth tubular neighborhood $\Omega$ of $M_{0}$. The manifold fitting problem is to find an embedding $F: M \rightarrow \mathbb{R}^{n}$ such that $F(M)$ is a good approximation to $M_{0}$ in the sense of least squares.

This situation occurs in a variety of contexts such as medical imaging $[2,14,16,17$, 18], geography [1], computer graphics and vision [5, 6, 9, 10, 15, 19, 22], and mechanical engineering $[12,13,20]$. In this setting, a scanning device is used to collect a set of points on or near the surface of an object, and the goal is to reconstruct the surface of the object from that point set. Viewing the surface as an embedding of a 2-dimensional manifold into $\mathbb{R}^{3}$ reduces the reconstruction problem to a special case of the manifold fitting problem.

Our approach to the manifold fitting problem is to replace the dataset $\mathbf{Y}$ by a smooth probability density $p$, which we assume has support in $\Omega$. We measure the goodness of fit 
of the embedding $F$ by the expected square of the distance to $F(M)$ :

$$
E_{d}[F]=\frac{1}{2} \int_{\mathbb{R}^{n}}\left|\mathbf{x}-F\left(\lambda_{F}(\mathbf{x})\right)\right|^{2} p(\mathbf{x}) d \mathbf{x},
$$

where the projection index $\lambda_{F}: \mathbb{R}^{n} \rightarrow M$ is the map that assigns to each point $\mathbf{x} \in \mathbb{R}^{n}$ a point in $M$ such that $F\left(\lambda_{F}(\mathbf{x})\right)$ realizes the distance from $\mathbf{x}$ to $F(M)$. This reduces the fitting problem to the problem of finding local minima of this functional.

The critical points of $E_{d}$ are called principal embeddings. The motivation for this name comes from the work by Hastie and Stuetzle [7] where the term of principal curve was coined to formalize the notion of "a curve passing through the middle of a dataset." Principal curves generalize the concept of linear principal components of a probability distribution, that is, straight lines that fit the distribution by least squares.

Duchamp and Stuetzle [4] showed that every critical curve of $E_{d}$ is a saddle point, and in Section 3 we generalize this result to arbitrary manifolds. Therefore, any method which seeks to minimize $E_{d}$ is guaranteed to fail.

We address this problem by adding a "regularizing term" to $E_{d}$. There are a number of possible terms, but a particularly simple one is the harmonic energy of the embedding $F$, which is defined by the expression

$$
E_{h}[F]=\frac{1}{2} \int_{M} g^{i j}\left\langle\frac{\partial F}{\partial x^{i}}, \frac{\partial F}{\partial x^{j}}\right\rangle d v o l_{g}
$$

where $g$ is a Riemannian metric on $M$ and $\langle\cdot, \cdot\rangle$ denotes the Euclidean metric on $\mathbb{R}^{n}$. Thus, we set

$$
E[F]=E_{d}[F]+k E_{h}[F]
$$

where $k>0$ is a suitably chosen parameter, which we call the spring constant, and we seek embeddings that are local minima of $E$. In Section 4 we establish sufficient conditions for the existence of a local minimum of this functional. Roughly speaking, the functional $E$ will have a local minimum whenever the spring constant $k$ is sufficiently big.

Our assumption that the support of $p$ lies in a tubular neighborhood of $M_{0}$ permits to limit the class of embeddings $F: M \rightarrow \mathbb{R}^{n}$ to the class of $\Omega$-regular embeddings. We say that $\mathbf{x} \in \mathbb{R}^{n}$ is a point of ambiguity if its distance to $F(M)$ is realized by more than one point in $F(M)$.

Definition 1.1. Let $\Omega \subset \mathbb{R}^{n}$ be a closed, smoothly bounded region. An embedding $F$ is $\Omega$-regular or regular with respect to $\Omega$ if it satisfies the following conditions:

(i) $F(M)$ is contained in the interior of $\Omega$,

(ii) $\Omega$ does not contain any point of ambiguity of $F(M)$,

(iii) the projection index $\lambda_{F}: \Omega \rightarrow M$ is a smooth $(n-m)$-disk bundle, and its restriction to the boundary $\lambda_{F}: \partial \Omega \rightarrow M$ is a smooth $(n-m-1)$-sphere bundle, 
(iv) for all $x \in M$, the disk $\lambda_{F}^{-1}(x)$ and $\partial \Omega$ have transverse intersection; that is, for all $\mathbf{x} \in \lambda_{F}^{-1}(x) \cap \Omega$,

$$
T_{\mathbf{x}} \lambda_{F}^{-1}(x)+T_{\mathbf{x}} \partial \Omega=T_{\mathbf{x}} \mathbb{R}^{n}
$$

We denote the class of $\Omega$-regular embeddings by $\mathscr{E}_{\Omega}$. We will henceforth assume that $\Omega$ is a fixed region that supports at least one $\Omega$-regular embedding, and we consider only probability densities whose support is contained in $\Omega$.

The paper is organized as follows. In Section 2 we give a jet bundle formulation to the theory of $\Omega$-regular embeddings. We believe that this is the natural framework to study the variational properties of the functional $E$. We start with some background material that will be needed in the following sections. We discuss isometric embeddings into $\mathbb{R}^{n}$ and normal coordinates of an embedded manifold $F: M \hookrightarrow \mathbb{R}^{n}$. Also, the concept of transverse statistics of a probability density is introduced.

In Section 3 we show that the distance functional does not have local minima within the class of $\Omega$-regular embeddings. This generalizes the results of Duchamp and Stuetzle on critical curves [4].

In Section 4 we lay the ground for the study of the general manifold fitting problem, paying particular attention to the variational properties of $E$ on the class of $\Omega$-regular embeddings. We characterize the critical points of $E$ in terms of harmonic maps and the extrinsic geometry of the embedded manifold $F(M)$. We also investigate conditions under which a critical embedding is a local minimum.

We conclude this paper with Section 5 where we summarize our results and explore topics of future research.

Notation 1.2. Einstein's summation convention is enforced throughout this paper. We use the first letters of the alphabet $a, b, c$, to denote indices varying between $m+1$ and $n$; the indices $i, j, k$ vary between 1 and $m$. Finally, Greek letters vary between 1 and $n$.

\section{The geometry of regular embeddings}

In this section we give a jet bundle formulation to the theory of $\Omega$-regular embeddings. We believe that this is the natural geometrical framework for the study of the variational properties of the functional $E$. We start with some background material that will be needed in the following sections, such as isometric embeddings into $\mathbb{R}^{n}$ and normal coordinates of an embedded manifold $F: M \hookrightarrow \mathbb{R}^{n}$. We conclude this section with the notion of transverse statistics of a probability density.

2.1. Embeddings into $\mathbb{R}^{n}$. In the forthcoming sections we deal with embedded manifolds in $\mathbb{R}^{n}$. In this section we introduce the basic definitions and notation we will use throughout.

Let $F: M \rightarrow \mathbb{R}^{n}$ be an $\Omega$-regular embedding. We denote by $N_{F(x)}$ the orthogonal complement of $F_{*}\left(T_{x} M\right)$ in $T_{F(x)} \mathbb{R}^{n}$, and define the bundle $N_{F}$ whose fiber at $x \in M$ is $N_{F(x)}$. We call $N_{F}$ the normal bundle of $F$. 
By parallel translation with respect to the Euclidean metric, we may identify the tangent space $T_{F(x)} \mathbb{R}^{n}$ with $\mathbb{R}^{n}$ itself. This gives rise to a bundle map

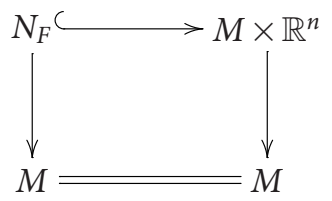

Recall that the projection index $\lambda_{F}: \mathbb{R}^{n} \rightarrow M$ is the map that assigns to each point $\mathbf{x} \in$ $\mathbb{R}^{n}$ a point $\lambda_{F}(\mathbf{x}) \in M$ such that $F\left(\lambda_{F}(\mathbf{x})\right)$ realizes the distance between $\mathbf{x}$ and $F(M)$. We consider the region $\Omega$ as an $(n-m)$-disk bundle with fiber at $x \in M$ given by $\Omega_{F(x)}=$ $\lambda_{F}^{-1}(x)$. Since $\Omega$ does not contain any point of ambiguity of $F(M)$, we can identify $\Omega$ with a subset of $N_{F}$ via the commutative diagram

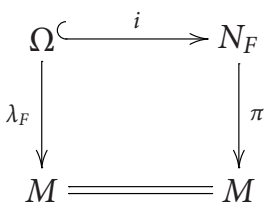

where $i$ is the map defined by

$$
i: \Omega \ni \mathbf{x} \longmapsto\left(\lambda_{F}(\mathbf{x}), \mathbf{x}-\lambda_{F}(\mathbf{x})\right) \in N_{F} \subset M \times \mathbb{R}^{n},
$$

and $\pi: N_{F} \rightarrow M$ is the canonical projection.

Let $h$ be the pullback along $F$ of the Euclidean metric on $\mathbb{R}^{n}$. We want to distinguish it from the background metric $g$ on $M$; that is, these are two different metrics defined on $M$.

Recall the definition of the second fundamental form $B: T M \times T M \rightarrow N_{F}$, which in local coordinates is given by

$$
\left\langle B\left(\frac{\partial}{\partial x^{i}}, \frac{\partial}{\partial x^{j}}\right), v\right\rangle=\left\langle\frac{\partial^{2} F}{\partial x^{i} \partial x^{j}}, v\right\rangle, \quad v \in N_{F} .
$$

We consider the linear selfadjoint operator $S: N_{F} \times T M \rightarrow T M$ defined by

$$
\langle S(v, X), Y\rangle=\langle B(X, Y), v\rangle, \quad X, Y \in T M, v \in N_{F} .
$$

This is called the shape operator of $F$. For convenience, we denote $S(v, X)$ by $S_{v} X$. Let $\nabla$ denote the covariant differentiation operator in $\mathbb{R}^{n}$. It is not hard to see from the definition of second fundamental form $B$ that $S_{v}$ satisfies

$$
\left\langle S_{v} X, Y\right\rangle=-\left\langle\nabla_{X} v, Y\right\rangle
$$

Remark 2.1. In local coordinates,

$$
\left(S_{v}\right)_{i}^{j}=h^{j k}\left\langle\frac{\partial^{2} F}{\partial x^{k} \partial x^{i}}, v\right\rangle .
$$


Example 2.2 (curves in $\mathbb{R}^{n}$ ). Let $M=S^{1}$. Denote by $\mathbf{T} \in C^{\infty}\left(S^{1}, \mathbb{R}^{n}\right)$ the unit tangent vector field along $F\left(S^{1}\right)$ and by $d \mathbf{T} / d s \in C^{\infty}\left(S^{1}, \mathbb{R}^{n}\right)$ its derivative with respect to arc length. Then, the shape operator $S_{v}$ in this case is a scalar multiplication by $\langle d \mathbf{T} / d s, v\rangle$. To check this, let $X=f \mathbf{T}, Y=g \mathbf{T} \in C^{\infty}\left(S^{1}, \mathbb{R}^{n}\right)$, and compute as follows:

$$
\begin{aligned}
\left\langle S_{v} X, Y\right\rangle & =\left\langle\nabla_{X} Y, v\right\rangle=\left\langle\nabla_{f \mathbf{T}}(g \mathbf{T}), v\right\rangle=\left\langle f \nabla_{\mathbf{T}}(g \mathbf{T}), v\right\rangle \\
& =\left\langle f g \nabla_{\mathbf{T}} \mathbf{T}, v\right\rangle+\langle f \mathbf{T}(g) \mathbf{T}, v\rangle \\
& =f g\left\langle\nabla_{\mathbf{T}} \mathbf{T}, v\right\rangle=\left\langle\left\langle\nabla_{\mathbf{T}} \mathbf{T}, v\right\rangle X, Y\right\rangle,
\end{aligned}
$$

which implies $S_{v} X=\langle d \mathrm{~T} / d s, v\rangle X$.

We close this section with the definition of normal connection $\nabla^{\perp}: T M \otimes N_{F} \rightarrow N_{F}$, given by

$$
(X, v) \longmapsto \nabla_{X}^{\perp} v \equiv \nabla_{X} v+S_{v} X
$$

It is easy to verify that $\nabla^{\perp}$ has all of the usual properties of a connection, that is, it is linear in $X$, additive in $v$, and satisfies the Leibniz rule,

$$
\nabla_{X}^{\perp} f v=f \nabla_{X}^{\perp} v+X(f) v, \quad f \in C^{\infty}(M) .
$$

2.2. Normal coordinates of an embedded manifold $M$. In this subsection we will be concerned with the geometry of tubular neighborhoods of a submanifold $M$ of Euclidean space. In this setting, the most convenient coordinates to use in computations are normal coordinates.

Definition 2.3. The normal coordinate map of $F$ is the map $\nu_{F}: N_{F} \rightarrow \mathbb{R}^{n}$ defined by the formula

$$
v_{F}(x, v)=F(x)+v .
$$

This is a diffeomorphism of a neighborhood of the zero section of $N_{F}$.

For future reference, we calculate the Jacobian determinant of the normal coordinate map. Let $\left\{e_{\alpha}\right\}$ be a locally defined adapted frame relative to $F(M)$ in $\mathbb{R}^{n}$, and let $\left\{\theta^{\alpha}\right\}$ denote the corresponding dual basis. We define a system of local coordinates $(x, v)$ in $N_{F}$ by writing $v \in N_{F(x)}$ as $v=v^{a} e_{a}(x)$. Consider the local frame $\left\{f_{\beta}\right\} \subset T N_{F}$ defined by

$$
f_{i}=e_{i}, \quad 1 \leq i \leq m, \quad f_{a}=\frac{\partial}{\partial v^{a}}, \quad m+1 \leq a \leq n .
$$

We compute the matrix representation of the differential $\nu_{F *}$ relative to the frames $\left\{e_{\alpha}\right\}$, $\left\{f_{\beta}\right\}$; that is, we compute the numbers $\theta^{\alpha}\left(d \nu_{F}\left(f_{\beta}\right)\right), 1 \leq \alpha, \beta \leq n$, where $d \nu_{F}: T N_{F} \rightarrow \mathbb{R}^{n}$ denotes the derivative of $\nu_{F}$. For $1 \leq i, j \leq m$, we get

$$
\theta^{i}\left(d \nu_{F}\left(f_{j}\right)\right)=\theta^{i}\left(d \nu_{F}\left(e_{j}\right)\right)=\theta^{i}\left(e_{j}(F+v)\right) .
$$

Since $e_{j}(F)=F_{*} e_{j}$ is identified with $e_{j}$ and since $e_{j}(v)=-S_{v} e_{j}+\nabla_{e_{j}}^{\perp} v$, we get

$$
\theta^{i}\left(d \nu_{F}\left(f_{j}\right)\right)=\theta^{i}\left(e_{j}-S_{\nu} e_{j}+\nabla_{e_{j}}^{\perp} v\right)
$$


Since $\theta^{i}\left(\nabla_{e_{j}}^{\perp}\right)=0$, we get

$$
\theta^{i}\left(d v_{F}\left(f_{j}\right)\right)=\theta^{i}\left(\left(I-S_{v}\right) e_{j}\right)
$$

We now compute $\theta^{i}\left(d \nu_{F}\left(f_{a}\right)\right), 1 \leq i \leq m, m+1 \leq a \leq n$. We get

$$
\theta^{i}\left(d v_{F}\left(f_{a}\right)\right)=\theta^{i}\left(\frac{\partial}{\partial v^{a}}(F+v)\right)
$$

Since $v=v^{b} e_{b}$

$$
\theta^{i}\left(d \nu_{F}\left(f_{a}\right)\right)=\theta^{i}\left(e_{a}\right)=0 .
$$

Finally, we compute $\theta^{a}\left(d v_{F}\left(f_{b}\right)\right)$ for $m+1 \leq a, b \leq n$ :

$$
\theta^{a}\left(d \nu_{F}\left(f_{b}\right)\right)=\theta^{a}\left(\frac{\partial}{\partial v^{b}}(F+v)\right) .
$$

After computing the derivative and simplifying, we get

$$
\theta^{a}\left(d \nu_{F}\left(f_{b}\right)\right)=\delta_{b}^{a}
$$

Using (2.15), (2.17), and (2.19), we see that the matrix representation of $v_{F *}$ relative to the frames $\left\{e_{\alpha}\right\},\left\{f_{\beta}\right\}$ is of the form

$$
\left[\nu_{F *}\right]=\left(\begin{array}{cc}
\theta^{i}\left(\left(I-S_{v}\right) e_{j}\right) & \theta^{a}\left(d \nu_{F}\left(e_{j}\right)\right) \\
0 & \delta_{b}^{a}
\end{array}\right),
$$

whose determinant is computed as $\operatorname{det}\left(I-S_{v}\right)$. Therefore, the Cartesian volume element $d \mathbf{x}$ in $\mathbb{R}^{n}$ becomes $\operatorname{det}\left(I-S_{v}\right) d v d v o l_{h}$, where $d v$ denotes the induced Euclidean volume element on $N_{F}$.

Example 2.4 (curves in $\mathbb{R}^{n}$ ). In this case, we have $\operatorname{det}\left(I-S_{v}\right)=1-\langle d \mathbf{T} / d s, v\rangle$. This follows from the computation in Example 2.2.

2.3. Regular jets. In our approach to the manifold fitting problem, we compute the expected value of the distance to $F(M)$ by making a change of variables to normal coordinates. This involves an integration over $M$ and an integration over the transverse disks to $F(M)$. When formal calculations are performed, it is natural to use the language of jets. In this way, the study of the regularity properties of $E_{d}$ reduces to the analysis of differentiable functions defined on a suitably chosen jet bundle.

Let $C^{\infty}\left(M, \mathbb{R}^{n}\right)$ denote the space of smooth maps from $M$ into $\mathbb{R}^{n}$. We consider the $p$ th jet space $J^{p}\left(M, \mathbb{R}^{n}\right)$. Recall that it consists of the equivalent classes $j_{x}^{p} F$ of maps $F \in C^{\infty}\left(M, \mathbb{R}^{n}\right)$ with the same Taylor series expansion up to order $p$ at $x \in M$. A local coordinate system of $J^{p}\left(M, \mathbb{R}^{n}\right)$ is defined as follows. Let $\left(x^{i}, y^{\alpha}\right)$ be a local coordinate system of $M \times \mathbb{R}^{n}$. Define the local coordinates $\left(x^{i}, y^{\alpha}, y_{i}^{\alpha}, \ldots, y_{i_{1} \cdots i_{p}}^{\alpha}\right)$ in $J^{p}\left(M, \mathbb{R}^{n}\right)$ by

$$
y^{\alpha}\left(j_{x}^{p} F\right)=y^{\alpha}(F(x)), \quad y_{i}^{\alpha}\left(j_{x}^{p} F\right)=\left.\frac{\partial F^{\alpha}}{\partial x^{i}}\right|_{x}, \ldots, y_{i_{1} \cdots i_{p}}^{\alpha}\left(j_{x}^{p} F\right)=\left.\frac{\partial^{p} F^{\alpha}}{\partial x^{i_{1}} \cdots \partial x^{i_{p}}}\right|_{x} .
$$


This is called the induced coordinate system on $J^{P}\left(M, \mathbb{R}^{n}\right)$. We are concerned with the open subset $J_{m}^{p}\left(M, \mathbb{R}^{n}\right)$ of $J^{p}\left(M, \mathbb{R}^{n}\right)$ of those $p$-jets $j_{x}^{p} F$ such that the linear map $F_{*}: T_{x} M \rightarrow \mathbb{R}^{n}$ has rank $m$. We define the notion of an $\Omega$-regular $p$-jet.

For $F \in \mathscr{E}_{\Omega}$ an $\Omega$-regular embedding, the distance functional $E_{d}[F]$ is defined by

$$
E_{d}[F]=\frac{1}{2} \int_{\Omega}\left|\mathbf{x}-F\left(\lambda_{F}(\mathbf{x})\right)\right|^{2} p(\mathbf{x}) d \mathbf{x}
$$

Observe that the vector $\mathbf{x}-F\left(\lambda_{F}(\mathbf{x})\right)$ is normal to $F(M)$ at $F\left(\lambda_{F}(\mathbf{x})\right)$. Denote by $\Omega_{F(x)}$ the connected component of $\Omega \cap\left(F(x)+N_{F(x)}\right)$ containing $F(x)$. By Fubini's theorem, we can compute $E_{d}[F]$ by integrating over the normal disks $\Omega_{F(x)}$, and then integrating over $M$. This gives

$$
E_{d}[F]=\frac{1}{2} \int_{M} \int_{\Omega_{F(x)}}|v|^{2} p(F+v) \operatorname{det}\left(I-S_{v}\right) d v d v o l_{h}
$$

Written in this way, the computation of $E_{d}[F]$ implies the knowledge of the tensor $S_{v}: T_{x} M \rightarrow T_{x} M$ at every point $x \in M$ and every direction $v \in \Omega_{F(x)}$. This is a pointwisedefined quantity which can be written in local coordinates as a function of up to secondorder derivatives of $F$ (Remark 2.1). We can consider $S_{v}$ as the value of a tensor-valued function locally defined near the two-jet $j_{x}^{2} F \in J_{m}^{2}\left(M, \mathbb{R}^{n}\right)$.

Recall that for $j_{x}^{p} F \in J_{m}^{p}\left(M, \mathbb{R}^{n}\right)$ the linear map $F_{*}: T_{x} M \rightarrow \mathbb{R}^{n}$ has rank $m$. Let $h\left(j_{x}^{p} F\right)$ denote the induced scalar product on $T_{x} M$ by $j_{x}^{p} F$ defined as

$$
\left(h\left(j_{x}^{p} F\right)\right)(X, Y)=\left.\left\langle F_{*} X, F_{*} Y\right\rangle\right|_{x}, \quad X, Y \in T_{x} M .
$$

In local coordinates, we write $h_{i j}=\left\langle\partial F /\left.\partial x^{i}\right|_{x}, \partial F /\left.\partial x^{j}\right|_{x}\right\rangle$ and let $h^{i j}$ denote the $(i, j)$-entry of the inverse matrix of $h\left(j_{x}^{p} F\right)$. Henceforth, we restrict ourselves to the case $p \geq 2$. For $v \in N_{F(x)}$, define the real numbers $\left(S_{v}\right)_{i}^{j}=h^{j k}\left\langle\partial^{2} F /\left.\partial x^{k} \partial x^{i}\right|_{x}, v\right\rangle$ and express any vector $X \in T_{x} M$ in terms of the local basis $\left\{\partial F /\left.\partial x^{1}\right|_{x}, \ldots, \partial F /\left.\partial x^{m}\right|_{x}\right\}$, as $X=X^{i} \partial /\left.\partial x^{i}\right|_{x}$. We define the linear map $S_{v}\left(\left(j_{x}^{p} F\right)\right): T_{x} M \rightarrow T_{x} M$ by

$$
\left(S_{v}\left(j_{x}^{p} F\right)\right) X=\left.\left(S_{v}\right)_{i}^{j} X^{i} \frac{\partial F}{\partial x^{j}}\right|_{x} .
$$

This is called the formal shape operator of $j_{x}^{p} F$ along $v \in N_{F(x)}$. It is a direct computation to show that this definition is coordinate independent. It follows from Remark 2.1 that at each $x \in M$, the local expression for the shape operator of $F$ coincides with its formal analog $S_{v}\left(j_{x}^{p} F\right)$. In particular, if we let $I: T_{x} M \rightarrow T_{x} M$ denote the identity map, the determinant $\operatorname{det}\left(I-S_{v}\left(j_{x}^{p} F\right)\right)$ is an invariantly defined smooth function on $J_{m}^{p}\left(M, \mathbb{R}^{n}\right)$.

We are now prepared to state our formal definition of $\Omega$-regular jets. 
Definition 2.5. $j_{x}^{p} F \in J_{m}^{p}\left(M, \mathbb{R}^{n}\right)$ is $\Omega$-regular if it satisfies the following conditions:

(i) $F(x)$ is in the interior of $\Omega$,

(ii) for all $v \in \mathbb{R}^{n}$ with $F(x)+v \in \Omega_{F(x)}$, $\operatorname{det}\left(I-S_{v}\left(j_{x}^{p} F\right)\right)>0$,

(iii) $\Omega_{F(x)}$ is diffeomorphic to an $(n-m)$-disk,

(iv) $\partial \Omega$ and $\Omega_{F(x)}$ intersect transversally.

The subset of $\Omega$-regular $p$-jets is denoted by $J_{\Omega}^{p}$. This is an open submanifold of $J_{m}^{p}(M$, $\mathbb{R}^{n}$ ) since the conditions (i) to (iv) above are open.

2.4. Transverse statistics. In this section we define various statistical quantities associated with a probability density $p$ and an $\Omega$-regular embedding $F$. For $x \in M$, the restriction of $p$ to $\Omega_{F(x)}$ is called the transverse density induced by $p$. We can do multivariate analysis on $\Omega_{F(x)}$ and talk about the mean and the covariance matrix of the transverse probability density. These concepts arise naturally as we use a system of normal coordinates of $F$ in computations.

We assume that for almost every $x \in M$ the set $\operatorname{supp}(p) \cap \Omega_{F(x)}$ has positive measure as a subset of the disk $\Omega_{F(x)}$.

Definition 2.6. The $k$ th transverse moment is the map $\Lambda_{k}: M \rightarrow \operatorname{Hom}\left(\mathbb{R}^{n} \times \cdots \times \mathbb{R}^{n}, \mathbb{R}\right)$ defined by

$$
\begin{aligned}
\Lambda_{0}(x) & =\int_{\Omega_{F(x)}} p(F(x)+v) d v, \quad k=0, \\
\Lambda_{k}\left(X_{1}, \ldots, X_{k}\right) & =\int_{\Omega_{F(x)}}\left\langle X_{1}, v\right\rangle \cdots\left\langle X_{k}, v\right\rangle p(F(x)+v) d v, \quad k>0,
\end{aligned}
$$

for $X_{1}, \ldots, X_{k} \in \mathbb{R}^{n}$.

Definition 2.7. The mean of the transverse density is the map

$$
\mu_{\perp}: M \longrightarrow \operatorname{Hom}\left(\mathbb{R}^{n}, \mathbb{R}\right)
$$

defined by

$$
\mu_{\perp}=\frac{\Lambda_{1}}{\Lambda_{0}}
$$

The covariance tensor of the transverse density is the symmetric map $\sigma_{\perp}^{2}: M \rightarrow$ $\operatorname{Hom}\left(\mathbb{R}^{n} \times \mathbb{R}^{n}, \mathbb{R}\right)$ defined by

$$
\sigma_{\perp}^{2}=\frac{\Lambda_{2}}{\Lambda_{0}}-\mu_{\perp} \otimes \mu_{\perp}
$$

Example 2.8 (radial distributions relative to a manifold $M$ ). Let $\Omega$ be a tubular neighborhood of a manifold $M$ with constant radius, and let $p$ be a probability density on $\Omega$ depending only on the distance to $M$. We call $p$ a radial density relative to $M$. In this case, the transverse mean $\mu_{\perp}$ vanishes identically and the transverse covariance tensor $\sigma_{\perp}^{2}$ is 
a constant multiple of the Euclidean scalar product restricted to the disks $\Omega_{F(x)}, x \in M$. To see this, write $v \in \Omega_{F(x)}$ in terms of the orthonormal frame $\left\{e_{1+m}, \ldots, e_{n}\right\} \subset N_{F(x)}$ as $v=v^{a} e_{a}$. Since $\Omega$ is a radially symmetric tubular neighborhood of $M$ and $p$ depends only on the distance to $M$, all of the transverse integrals

$$
\int_{\Omega_{F(x)}} p(F(x)+v) d v, \quad \int_{\Omega_{F(x)}} v^{a} p(F(x)+v) d v, \quad \int_{\Omega_{F(x)}} v^{a} v^{b} p(F(x)+v) d v
$$

are constant. In particular, those whose integrand depends on odd powers of $v^{a}$ vanish. To check this, change to polar coordinates $v=r \theta, r \geq 0, \theta \in S^{n-2}$ to compute them. Since $p(F(x)+v)$ is a function of $r$ only, integration over $S^{n-2}$ vanishes because the values of $v^{a}$ taken on one hemisphere are exactly canceled by the values taken on the antipodal hemisphere. Therefore, the transverse mean $\mu_{\perp}$ vanishes as well as the off-diagonal entries of the matrix representation of $\sigma_{\perp}^{2}$ with respect to the basis $\left\{e_{1+m}, \ldots, e_{n}\right\}$. Since $\Omega_{F(x)}$ is homothetic to the unit disk $D^{n-m}$ and $p$ depends only on $|v|$, all of the diagonal entries $\left(\sigma_{\perp}^{2}\right)^{a a}$ are the same. Consequently $\sigma_{\perp}^{2}$ is a constant multiple of the Euclidean scalar product restricted to $\Omega_{F(x)}$.

\section{Extremal properties of $E_{d}$}

Recall that for an $m$-dimensional compact orientable Riemannian manifold $M$ without boundary and an $\Omega$-regular embedding $F: M \rightarrow \mathbb{R}^{n}$, the distance functional is one half of the expected value of the square of distance to $F(M)$,

$$
E_{d}[F]=\frac{1}{2} \int_{\Omega}\left|\mathbf{x}-F\left(\lambda_{F}(\mathbf{x})\right)\right|^{2} p(\mathbf{x}) d \mathbf{x}
$$

where $\lambda_{F}$ is the projection index and $p$ is the probability density. As we mentioned in the introduction, this functional does not have local minima within the class of smooth $\Omega$ regular embeddings. In this section we prove this fact by studying the extremal properties of $E_{d}$.

3.1. Function spaces. Up to now the functional $E_{d}$ has been defined for smooth embeddings. To study its extremal properties we consider it as a functional defined on a Banach space. Since these properties can be formulated in terms of differential equations, it is convenient to consider Sobolev spaces for the domain of $E_{d}$.

For an $m$-dimensional compact Riemannian manifold $M$ without a boundary, we define the Sobolev spaces $\Gamma^{k}\left(M, \mathbb{R}^{n}\right), k=0,1, \ldots$, as follows. Let $\left\{U_{i}\right\}_{i \in I}$ be a finite cover of $M$ by local charts diffeomorphic to open bounded subsets of $\mathbb{R}^{m}$. Let $\left\{\varphi_{i}\right\}$ be a partition of unity subordinate to $\left\{U_{i}\right\}$. For $F \in C^{\infty}\left(M, \mathbb{R}^{n}\right)$, we let

$$
\left\|\varphi_{i} F\right\|_{k}^{2}=\sum_{|\alpha| \leq k} \int_{U_{i}}\left|D^{\alpha}\left(\varphi_{i} F\right)\right|^{2} d x
$$


be the square of the $k$-Sobolev norm of the vector-valued function $\varphi_{i} F: U_{i} \rightarrow \mathbb{R}^{n}$. Then $\Gamma^{k}\left(M, \mathbb{R}^{n}\right)$ is the completion of $C^{\infty}\left(M, \mathbb{R}^{n}\right)$ with respect to the norm

$$
\|F\|_{k}=\left(\sum_{i \in I}\left\|\varphi_{i} F\right\|_{k}^{2}\right)^{1 / 2}
$$

For $k \geq 0$, let $C^{k}\left(M, \mathbb{R}^{n}\right)$ be the Banach space of $C^{k}$ functions with the norm of the supreme. Sobolev's lemma establishes that the inclusion $\Gamma^{k+k_{0}}\left(M, \mathbb{R}^{n}\right) \hookrightarrow C^{k}\left(M, \mathbb{R}^{n}\right)$ is continuous, where $k_{0}=1+[(m-1) / 2]$ and $[(m-1) / 2]$ denotes the least integer greater than or equal to $(m-1) / 2$.

We extend the class of $\Omega$-regular embeddings $\mathscr{E}_{\Omega}$ to include those embeddings in $\Gamma^{k}\left(M, \mathbb{R}^{n}\right)$ satisfying conditions (i) through (iv) of Definition 1.1. We denote this subset of $\Gamma^{k}\left(M, \mathbb{R}^{n}\right)$ by $\mathscr{E}_{\Omega}^{k}$. For $k \geq 2+k_{0}$, this is an open subset of $\Gamma^{k}\left(M, \mathbb{R}^{n}\right)$. This follows from Sobolev's lemma and from the fact that the subset of $\Omega$-regular two-jets $J_{\Omega}^{2}$ is an open submanifold of $J^{2}\left(M, \mathbb{R}^{n}\right)$; that is, any embedding $F \in \mathscr{E}_{\Omega}^{k}$ has a neighborhood $U \subset \Gamma^{k}\left(M, \mathbb{R}^{n}\right)$ such that for all $\tilde{F} \in U$, the difference between the Taylor series expansions up two second order of $F$ and $\tilde{F}$ is so small that $\tilde{F}$ also satisfies conditions (i) to (iv) of Definition 1.1.

Lemma 3.1. Let $l$ and $q$ be positive integers and let $k>m$. Then the map $C: \mathscr{C}_{\Omega}^{k+2} \times$ $C^{k+l+1}\left(J_{\Omega}^{2}, \mathbb{R}^{q}\right) \rightarrow \Gamma^{k}\left(M, \mathbb{R}^{q}\right)$ defined by

$$
(C(F, g))(x)=g\left(j_{x}^{2} F\right)
$$

is a well-defined $C^{l}$ map.

Proof. We define some quantities first. For $V \in C^{\infty}\left(M, \mathbb{R}^{n}\right)$, let $\delta^{2} V: M \rightarrow T J_{\Omega}^{2}$ be the map defined in local coordinates by the formula

$$
\delta^{2} V=\sum_{r=1}^{2} \frac{\partial^{r} V^{\alpha}}{\partial x^{i_{1}} \cdots \partial x^{i_{r}}} \frac{\partial}{\partial y_{i_{1} \cdots i_{r}}^{\alpha}} .
$$

For $j=0,1, \ldots, l$, let

$$
d^{(j)} g: J_{\Omega}^{2} \longrightarrow \operatorname{Hom}(\overbrace{T J_{\Omega}^{2} \times \cdots \times T J_{\Omega}^{2}}^{j \text { times }}, \mathbb{R}^{q})
$$

be the $j$ th total derivative of $g$. We define the map

$$
C^{(j)}: \mathscr{E}_{\Omega} \times C^{k+l+1}\left(J_{\Omega}^{2}, \mathbb{R}^{q}\right) \longrightarrow \operatorname{Hom}(\overbrace{C^{\infty}\left(M, \mathbb{R}^{n}\right) \times \cdots \times C^{\infty}\left(M, \mathbb{R}^{n}\right)}^{j \text { times }}, C^{k+l+1-j}\left(M, \mathbb{R}^{q}\right))
$$

given by

$$
\left(C^{(j)}(F, g)\right)\left(V_{1}, \ldots, V_{j}\right)_{x}=\left(d^{(j)} g\left(j_{x}^{2} F\right)\right)\left(\delta^{2} V_{1}, \ldots, \delta^{2} V_{j}\right), \quad V_{i} \in C^{\infty}\left(M, \mathbb{R}^{n}\right) .
$$


We denote by $\mathbf{B}\left(\Gamma^{k+2}\left(M, \mathbb{R}^{n}\right) \times \cdots \times \Gamma^{k+2}\left(M, \mathbb{R}^{n}\right), \Gamma^{k}\left(M, \mathbb{R}^{q}\right)\right)$ the Banach space of bounded operators between $\Gamma^{k+2}\left(M, \mathbb{R}^{n}\right) \times \cdots \times \Gamma^{k+2}\left(M, \mathbb{R}^{n}\right)$ and $\Gamma^{k}\left(M, \mathbb{R}^{q}\right)$.

The proof of Lemma 3.1 consists of the following steps.

(1) For $j=0,1, \ldots, l$, we show that the map $C^{(j)}$ extends to a $\mathbf{B}\left(\Gamma^{k+2}\left(M, \mathbb{R}^{n}\right) \times \cdots \times\right.$ $\left.\Gamma^{k+2}\left(M, \mathbb{R}^{n}\right), \Gamma^{k}\left(M, \mathbb{R}^{q}\right)\right)$-valued function defined on $\mathscr{C}_{\Omega}^{k+2} \times C^{k+l+1}\left(J_{\Omega}^{2}, \mathbb{R}^{q}\right)$. We also show that for fixed $g$, the map

$$
C^{(j)}(\cdot, g): \mathscr{E}_{\Omega}^{k+2} \longrightarrow \mathbf{B}\left(\Gamma^{k+2}\left(M, \mathbb{R}^{n}\right) \times \cdots \times \Gamma^{k+2}\left(M, \mathbb{R}^{n}\right), \Gamma^{k}\left(M, \mathbb{R}^{q}\right)\right)
$$

is continuous.

(2) For fixed $g$, we show that $C^{(j)}(\cdot, g)$ is the $j$ th derivative of $C(\cdot, g)$.

(3) Finally, we show that $C$ is a $C^{l}$ map.

For the proof of the lemma, a formula to compute the derivatives of $C^{(j)}(F, g)$ in local coordinates is needed. Let $\left(U \times \mathbb{R}^{n} ; x^{i}, y^{\gamma}\right)$ be a local coordinate system on $M \times \mathbb{R}^{n}$, and let $\left(W ; x^{i}, y^{\gamma}, y_{i}^{\gamma}, \ldots, y_{i_{1} \cdots i_{2}}^{\gamma}\right)$ be the induced local coordinate system in $J_{\Omega}^{2}$. For a multi-index $\alpha=\left(a_{1}, \ldots, a_{m}\right), a_{i} \in \mathbb{N}$, let $|\alpha|=\sum_{i=1}^{m} a_{i}$. Denote the $\alpha$-derivative of a smooth function $f \in C^{\infty}(U)$ by

$$
D^{\alpha} f=\frac{\partial^{|\alpha|} f}{\partial\left(x^{1}\right)^{a_{1}} \cdots \partial\left(x^{m}\right)^{a_{m}}} .
$$

For an embedding $F \in \mathscr{E}_{\Omega}$ and a function $g \in C^{k+l+1}\left(J_{\Omega}^{2}, \mathbb{R}^{q}\right)$, we compute

$$
\begin{gathered}
\sum_{|\alpha| \leq k} D^{\alpha}\left(\left(C^{(j)}(F, g)\right)\left(V_{1}, \ldots, V_{j}\right)_{x}\right) \\
=\sum_{i=0}^{[k / 2]-1} \sum_{\left|\alpha_{0}+\alpha_{1}+\cdots+\alpha_{j}\right| \leq k+2-i} g_{i, \alpha_{0} \cdots \alpha_{j}}\left(j_{x}^{i+2} F\right)\left(D^{\alpha_{0}} F, D^{\alpha_{1}} V_{1}, \ldots, D^{\alpha_{j}} V_{j}\right), \\
V_{1}, \ldots, V_{j} \in C^{\infty}\left(M, \mathbb{R}^{n}\right),
\end{gathered}
$$

where the functions $g_{i, \alpha_{0} \cdots \alpha_{j}}$ are $\operatorname{Hom}(\overbrace{\mathbb{R}^{n} \times \cdots \times \mathbb{R}^{n}}^{j \text { times }}, \mathbb{R}^{q})$-valued $C^{1}$ functions defined on $W$. This formula follows by inductively computing the derivatives of $\left(C^{(j)}(F, g)\right)$ $\left(V_{1}, \ldots, V_{j}\right)$ using the definition (3.8). For $j=1$, we get

$$
\left(C^{(1)}(F, g)\right)(V)_{x}=\sum_{r=1}^{2} \frac{\partial g\left(j_{x}^{2} F\right)}{\partial y_{i_{1}}^{\gamma} \cdots i_{r}} \frac{\partial^{r} V^{\gamma}}{\partial x^{i_{1}} \cdots \partial x^{i_{r}}} .
$$

By the Leibniz rule,

$$
\sum_{|\alpha| \leq k} D^{\alpha}\left(C^{(1)}(F, g)\right)(V)_{x}=\sum_{r=1}^{2} \sum_{|\alpha| \leq k} \sum_{\beta \leq \alpha} c_{\alpha \beta} D^{\alpha-\beta}\left(\frac{\partial g\left(j_{x}^{2} F\right)}{\partial y_{i_{1} \cdots i_{r}}^{\gamma}}\right) D^{\alpha}\left(\frac{\partial^{r} V^{\gamma}}{\partial x^{i_{1}} \cdots \partial x^{i_{r}}}\right),
$$


where $\beta \leq \alpha$ means $b_{i} \leq a_{i}$ for $1 \leq i \leq m$, and the numbers $c_{\alpha \beta}$ are positive integers whose exact value is irrelevant to our present needs. We compute the $(\alpha-\beta)$ th derivative of $\partial g\left(j_{x}^{2} F\right) / \partial y_{i_{1} \ldots i_{r}}^{\gamma}$ by using the chain rule, and then writing the result in the form

$$
\sum_{|\alpha| \leq k} D^{\alpha}\left(C^{(1)}(F, g)\right)(V)_{x}=\sum_{i=0}^{[k / 2]-1} \sum_{|\alpha+\beta| \leq k+2-i} g_{i, \alpha \beta}\left(j_{x}^{i+2} F\right)\left(D^{\alpha} F, D^{\beta} V\right),
$$

where the $g_{i+2, \alpha \beta}$ are $\operatorname{Hom}\left(\mathbb{R}^{n} \times \mathbb{R}^{n}, \mathbb{R}^{q}\right)$-valued functions defined on $W$. These are at least $C^{1}$ since they are given in terms of the first $k+1$ derivatives of $g$, which is $C^{k+l+1}$.

Step 1. Let $F \in \mathscr{E}_{\Omega}^{k+2}$ and $g \in C^{k+l+1}\left(J_{\Omega}^{2}, \mathbb{R}^{q}\right)$. Consider a sequence $\left\{F_{l}\right\} \subset \mathscr{E}_{\Omega}$ converging to $F$ in the $\Gamma^{k+2}\left(M, \mathbb{R}^{n}\right)$ topology. We show that for any set of vectors $V_{1}, \ldots, V_{j} \in$ $C^{\infty}\left(M, \mathbb{R}^{n}\right)$, we have $\left(C^{(j)}\left(F_{l}, g\right)\right)\left(V_{1}, \ldots, V_{j}\right) \in \Gamma^{k}\left(M, \mathbb{R}^{q}\right)$. Moreover, we also show that $C^{(j)}\left(F_{l}, g\right)$ defines a bounded operator in $\mathbf{B}\left(\Gamma^{k+2}\left(M, \mathbb{R}^{n}\right) \times \cdots \times \Gamma^{k+2}\left(M, \mathbb{R}^{n}\right), \Gamma^{k}\left(M, \mathbb{R}^{q}\right)\right)$, and that the sequence $\left\{C^{(j)}\left(F_{l}, g\right)\right\} \subset \mathbf{B}\left(\Gamma^{k+2}\left(M, \mathbb{R}^{n}\right) \times \cdots \times \Gamma^{k+2}\left(M, \mathbb{R}^{n}\right), \Gamma^{k}\left(M, \mathbb{R}^{q}\right)\right)$ is Cauchy. By completeness of the operator space, this sequence converges and we define its limit as $C^{(j)}(F, g)$. This defines $C^{(j)}(F, g)$ as a bounded operator in $\mathbf{B}\left(\Gamma^{k+2}\left(M, \mathbb{R}^{n}\right) \times \cdots \times\right.$ $\left.\Gamma^{k+2}\left(M, \mathbb{R}^{n}\right), \Gamma^{k}\left(M, \mathbb{R}^{q}\right)\right)$. In particular, the map $C^{(j)}(\cdot, g): \mathscr{E}_{\Omega}^{k+2} \rightarrow \mathbf{B}\left(\Gamma^{k+2}\left(M, \mathbb{R}^{n}\right) \times \cdots \times\right.$ $\left.\Gamma^{k+2}\left(M, \mathbb{R}^{n}\right), \Gamma^{k}\left(M, \mathbb{R}^{q}\right)\right)$ is continuous for fixed $g$, by construction.

To show that $\left(C^{(j)}\left(F_{l}, g\right)\right)\left(V_{1}, \ldots, V_{j}\right) \in \Gamma^{k}\left(M, \mathbb{R}^{q}\right)$, we apply formula (3.11) and show that it defines a square integrable function on $U \subset \mathbb{R}^{m}$. Notice that the order $i+2$ of the jets $j_{x}^{i+2} F_{l}$ satisfies $i+2 \leq[k / 2]-1+2$, since the sum over $i$ runs up to $[k / 2]-1$. Because $k>2$, we have that $[k / 2]-1+2 \leq k+2-1-[(m-1) / 2]=k+2-k_{0}$, where $k_{0}=1+[(m-1) / 2]$. By Sobolev's lemma, the sequence $\left\{F_{l}\right\}$ is a converging sequence in $C^{k+2-k_{0}}\left(M, \mathbb{R}^{n}\right)$. Consequently, the closure of the set $\left\{j_{x}^{i+2} F_{l} \in J_{\Omega}^{i+2}: l \in \mathbb{N}, x \in M\right\}$ is contained in a compact subset $K \subset J_{\Omega}^{i+2}$, since in any local coordinate chart, the Taylor series expansion of the $F_{l}$ 's is bounded. In particular, the functions $g_{i, \alpha_{0} \cdots \alpha_{j}}\left(j_{x}^{i+2} F_{l}\right)$ are bounded on $U \subset \mathbb{R}^{m}$. On the other hand, suppose that two multi-indices $\alpha_{s}$ and $\alpha_{t}$ in (3.11) satisfied $\left|\alpha_{s}\right|,\left|\alpha_{t}\right|>k+2-k_{0}$. We would have $\left|\alpha_{s}+\alpha_{t}\right|=\left|\alpha_{s}\right|+\left|\alpha_{t}\right|>2(k+$ $\left.2-k_{0}\right)$. Since these indices vary between $0 \leq\left|\alpha_{s}+\alpha_{t}\right| \leq k+2-i$, and $i$ varies within $0 \leq i \leq[k / 2]-1$, we would have $2\left(k+2-k_{0}\right)<k+2-([k / 2]-1)$. A computation shows that this contradicts our assumption on the value of $k$. As a conclusion, at most one multi-index $\alpha_{s}$ is strictly greater than $k+2-k_{0}$ in magnitude. By Sobolev's lemma, we have that $j$ out of $D^{\alpha_{0}} F_{l}, D^{\alpha_{1}} V_{1}, \ldots, D^{\alpha_{j}} V_{j}$ are continuous functions on $U \subset \mathbb{R}^{m}$. This shows that (3.11) is square integrable. By defining a partition of unity subordinated to a finite cover of $M$ by local charts, we conclude that $C^{(j)}\left(F_{l}, g\right)\left(V_{1}, \ldots, V_{j}\right) \in \Gamma^{k}\left(M, \mathbb{R}^{q}\right)$.

We next show that $C^{(j)}\left(F_{l}, g\right) \in \mathbf{B}\left(\Gamma^{k+2}\left(M, \mathbb{R}^{n}\right) \times \cdots \times \Gamma^{k+2}\left(M, \mathbb{R}^{n}\right), \Gamma^{k}\left(M, \mathbb{R}^{q}\right)\right)$ for all $l \in \mathbb{N}$, and that $\left\{C^{(j)}\left(F_{l}, g\right)\right\}$ is a Cauchy sequence. We start by estimating the difference $\left(C^{(j)}\left(F_{l}, g\right)-C^{(j)}\left(F_{m}, g\right)\right)\left(V_{1}, \ldots, V_{j}\right)$ in the $\Gamma^{k}$-norm. We write

$$
\begin{aligned}
g_{i, \alpha_{0} \cdots \alpha_{j}} & \left(j_{x}^{i+2} F_{l}\right)\left(D^{\alpha_{0}} F_{l}, D^{\alpha_{1}} V_{1}, \ldots, D^{\alpha_{j}} V_{j}\right)-g_{i, \alpha_{0} \cdots \alpha_{j}}\left(j_{x}^{i+2} F_{m}\right)\left(D^{\alpha_{0}} F_{m}, D^{\alpha_{1}} V_{1}, \ldots, D^{\alpha_{j}} V_{j}\right) \\
= & g_{i, \alpha_{0} \cdots \alpha_{j}}\left(j_{x}^{i+2} F_{l}\right)\left(D^{\alpha_{0}} F_{l}-D^{\alpha_{0}} F_{m}, D^{\alpha_{1}} V_{1}, \ldots, D^{\alpha_{j}} V_{j}\right) \\
& +\left(g_{i, \alpha_{0} \cdots \alpha_{j}}\left(j_{x}^{i+2} F_{l}\right)-g_{i, \alpha_{0} \cdots \alpha_{j}}\left(j_{x}^{i+2} F_{m}\right)\right)\left(D^{\alpha_{0}} F_{m}, D^{\alpha_{1}} V_{1}, \ldots, D^{\alpha_{j}} V_{j}\right) .
\end{aligned}
$$


Let $W$ be a local chart of $J_{\Omega}^{i+2}$ induced by the chart $U \subset \mathbb{R}^{m}$. Without loss of generality we assume that $W$ is a convex subset of Euclidean space. By the mean value theorem applied to the $C^{2}$ function $g_{i, \alpha_{0} \cdots \alpha_{j}}: W \rightarrow \operatorname{Hom}\left(\mathbb{R}^{n} \times \cdots \times \mathbb{R}^{n}, \mathbb{R}^{q}\right)$, there exists $t \in[0,1]$ such that

$$
\begin{aligned}
& \left(g_{i, \alpha_{0} \cdots \alpha_{j}}\left(j_{x}^{i+2} F_{l}\right)-g_{i, \alpha_{0} \cdots \alpha_{j}}\left(j_{x}^{i+2} F_{m}\right)\right) \\
& \quad=d g_{i, \alpha_{0} \cdots \alpha_{j}}\left((1-t) j_{x}^{i+2} F_{l}+t j_{x}^{i+2} F_{m}\right)\left(\delta^{i+2} F_{l}-\delta^{i+2} F_{m}\right),
\end{aligned}
$$

where $d g_{i, \alpha_{0} \cdots \alpha_{j}}: T W \rightarrow \operatorname{Hom}\left(\mathbb{R}^{n} \times \cdots \times \mathbb{R}^{n}, \mathbb{R}^{q}\right)$ denotes the differential of $g_{i, \alpha_{0} \cdots \alpha_{j}}$ and the map $\delta^{i+2} F_{l}: U \rightarrow T W$ is defined by (3.5). By hypothesis, the sequence $\left\{F_{l}\right\} \subset \Gamma^{k+2}(M$, $\left.\mathbb{R}^{n}\right)$ is Cauchy and by Sobolev's lemma, it is also Cauchy in $C^{k+2-k_{0}}\left(M, \mathbb{R}^{n}\right)$. Therefore, since $i+2 \leq k+2-k_{0}$, the convex hull of the set $\left\{j_{x}^{i+2} F_{l} \in J_{\Omega}^{i+2}: l \in \mathbb{N}, x \in M\right\}$ is contained in a compact subset $K \subset J_{\Omega}^{i+2}$, and then the maps $g_{i, \alpha_{0} \cdots \alpha_{j}}, d g_{i, \alpha_{0} \cdots \alpha_{j}}$ are bounded on $W$. We now use (3.15) and (3.16) to obtain a global estimate in the $\Gamma^{k}$-norm of the difference $\left(C^{(j)}\left(F_{l}, g\right)-C^{(j)}\left(F_{m}, g\right)\right)\left(V_{1}, \ldots, V_{j}\right)$ using a partition of unity. This gives

$$
\begin{aligned}
\|\left(C^{(j)}\right. & \left.\left(F_{l}, g\right)-C^{(j)}\left(F_{m}, g\right)\right)\left(V_{1}, \ldots, V_{j}\right) \|_{k} \\
\leq & c_{1}\left\|F_{l}-F_{m}\right\|_{k+2}\left\|V_{1}\right\|_{k+2} \cdots\left\|V_{j}\right\|_{k+2} \\
& +c_{2}\left\|F_{l}-F_{m}\right\|_{C^{k+2-k_{0}}}\left\|F_{m}\right\|_{k+2}\left\|V_{1}\right\|_{k+2} \cdots\left\|V_{j}\right\|_{k+2},
\end{aligned}
$$

for positive $c_{1}, c_{2}$. Since $\left\{\left\|F_{m}\right\|_{k+2}\right\} \subset \mathbb{R}$ is a bounded sequence of real numbers and the inclusion $\Gamma^{k+2}\left(M, \mathbb{R}^{n}\right) \hookrightarrow C^{k+2-k_{0}}\left(M, \mathbb{R}^{n}\right)$ is continuous, the last term above is bounded by a positive multiple of $\left\|F_{l}-F_{m}\right\|_{k+2}\left\|V_{1}\right\|_{k+2} \cdots\left\|V_{j}\right\|_{k+2}$. This shows that $C^{(j)}\left(F_{l}, g\right)-$ $C^{(j)}\left(F_{m}, g\right)$ is a bounded operator in $\mathbf{B}\left(\Gamma^{k+2}\left(M, \mathbb{R}^{n}\right) \times \cdots \times \Gamma^{k+2}\left(M, \mathbb{R}^{n}\right), \Gamma^{k}\left(M, \mathbb{R}^{q}\right)\right)$ whose operator norm is bounded by a positive multiple of $\left\|F_{l}-F_{m}\right\|_{k+2}$. This shows that the sequence

$$
\left\{C^{(j)}\left(F_{l}, g\right)-C^{(j)}\left(F_{m}, g\right)\right\} \subset \mathbf{B}\left(\Gamma^{k+2}\left(M, \mathbb{R}^{n}\right) \times \cdots \times \Gamma^{k+2}\left(M, \mathbb{R}^{n}\right), \Gamma^{k}\left(M, \mathbb{R}^{q}\right)\right)
$$

is Cauchy. This concludes the proof of Step 1.

Step 2. Fix $g \in C^{k+l+1}\left(J_{\Omega}^{2}, \mathbb{R}^{q}\right)$. For $j=1$, we show that the limit

$$
\lim _{t \rightarrow 0}\left\|\frac{C(F+t V, g)-C(F, g)}{t}-C^{(1)}(F, g) V\right\|_{k}=0
$$

for all $F \in \mathscr{E}_{\Omega}, V \in C^{\infty}\left(M, \mathbb{R}^{n}\right)$. Since the map

$$
C^{(1)}(\cdot, g): \mathscr{E}_{\Omega}^{k+2} \longrightarrow \mathbf{B}\left(\Gamma^{k+2}\left(M, \mathbb{R}^{n}\right), \Gamma^{k}\left(M, \mathbb{R}^{q}\right)\right)
$$

is continuous, we conclude that $C^{(1)}(\cdot, g)$ is the derivative of $C(\cdot, g)[11$, page 37].

To prove (3.19), we consider a cover of $M$ by local charts. In each of these charts $U \subset$ $\mathbb{R}^{m}$, we have the pointwise limit

$$
\lim _{t \rightarrow 0} \sum_{|\alpha| \leq k} D^{\alpha}\left(\frac{g\left(j_{x}^{2}(F+t V)\right)-g\left(j_{x}^{p} F\right)}{t}-d g\left(j_{x}^{2} F\right) \cdot \delta^{2} V\right)=0 .
$$


We can write the quotient $\left(g\left(j_{x}^{2}(F+t V)\right)-g\left(j_{x}^{2} F\right)\right) / t$ by applying the mean value theorem to the function $\phi(t)=g\left(j_{x}^{2}(F+t V)\right)$. We get

$$
\frac{g\left(j_{x}^{2}(F+t V)\right)-g\left(j_{x}^{2} F\right)}{t}=d g\left(j_{x}^{2}\left(F+t_{1} V\right)\right) \cdot \delta^{2} V,
$$

for some $\left|t_{1}\right| \leq|t|$. We apply the Lebesgue dominated convergence theorem to the sequence

$$
\left\{\sum_{|\alpha| \leq k} D^{\alpha}\left(\frac{g\left(j_{x}^{2}(F+t V)\right)-g\left(j_{x}^{2} F\right)}{t}-d g\left(j_{x}^{2} F\right) \cdot \delta^{2} V\right)\right\}_{t \in I} \subset \Gamma^{0}\left(U, \mathbb{R}^{q}\right)
$$

to show that the limit (3.21) holds in the $\Gamma^{0}$-norm. For this, it suffices to find a function $G \in \Gamma^{0}(U, \mathbb{R})$ such that

$$
\left|\sum_{|\alpha| \leq k} D^{\alpha} d g\left(j_{x}^{2}(F+t V)\right) \cdot \delta^{2} V\right| \leq G
$$

for all $t \in I$, since by (3.22) we will have

$$
\left|\sum_{|\alpha| \leq k} D^{\alpha}\left(\frac{g\left(j_{x}^{2}(F+t V)\right)-g\left(j_{x}^{2} F\right)}{t}-d g\left(j_{x}^{2} F\right) \cdot \delta^{2} V\right)\right| \leq 2 G .
$$

To construct such a function $G$, we give a uniform estimate on $t$ of

$$
\left|\sum_{|\alpha| \leq k} D^{\alpha} d g\left(j_{x}^{2}(F+t V)\right) \cdot \delta^{2} V\right| .
$$

By formula (3.11), we have

$$
\begin{aligned}
\sum_{|\alpha| \leq k} D^{\alpha} d g\left(j_{x}^{2}(F+t V)\right) \cdot \delta^{2} V \\
\quad=\sum_{i=0}^{[k / 2]-1} \sum_{|\alpha+\beta| \leq k+2-i} g_{i, \alpha \beta}\left(j_{x}^{i+2}(F+t V)\right)\left(D^{\alpha}(F+t V), D^{\beta} V\right) .
\end{aligned}
$$

Since $t$ varies within a small interval, the set $\left\{j_{x}^{i+2}(F+t V) \in J_{\Omega}^{i+2}: t \in I, x \in M\right\}$ is contained in a compact subset $K \subset J_{\Omega}^{i+2}$, and then the functions $g_{i, \alpha \beta}$ are bounded on it. We estimate

$$
\begin{aligned}
\left|\sum_{|\alpha| \leq k} D^{\alpha} d g\left(j_{x}^{2}(F+t V)\right) \cdot \delta^{2} V\right| & \leq c \sum_{i=0}^{[k / 2]-1} \sum_{|\alpha+\beta| \leq k+2-i}\left|D^{\alpha}(F+t V)\right|\left|D^{\beta} V\right| \\
& \leq c \sum_{i=0}^{[k / 2]-1} \sum_{|\alpha+\beta| \leq k+2-i}\left(\left|D^{\alpha} F\right|+\left|D^{\alpha} V\right|\right)\left|D^{\beta} V\right|,
\end{aligned}
$$


for some $c>0$. Set

$$
G=c \sum_{i=0}^{[k / 2]-1} \sum_{|\alpha+\beta| \leq k+2-i}\left(\left|D^{\alpha} F\right|+\left|D^{\alpha} V\right|\right)\left|D^{\beta} V\right| .
$$

A computation shows that at least one of the multi-indices $\alpha, \beta$ is less than or equal to $k+2-k_{0}$. By Sobolev's lemma, at least one of $D^{\alpha} F, D^{\beta} V$ is a continuous function. This shows that $G \in \Gamma^{0}(U, \mathbb{R})$. Therefore, the limit (3.21) holds in $\Gamma^{0}\left(U, \mathbb{R}^{q}\right)$ for every local chart $U$ of $M$. A similar argument shows that $C^{(j)}(\cdot, g)$ is the $j$-derivative of $C(\cdot, g)$. This proves Step 2.

Step 3. To show that the map $C$ is $C^{l}$, we first note that for $g_{1}, g_{2} \in C^{k+l+1}\left(J_{\Omega}^{2}, \mathbb{R}^{q}\right)$ we have $g_{1}\left(j_{x}^{2} F\right)+g_{2}\left(j_{x}^{2} F\right)=\left(g_{1}+g_{2}\right)\left(j_{x}^{2} F\right)$; that is, the map $C(F, g)$ is linear in $g$ and, since it is a bounded operator for fixed $F$, it is also smooth. We have also shown that $C^{(j)}(F, g)$ is continuous in $F$ for fixed $g$, therefore it suffices to show that it is jointly continuous in $F$ and $g$.

Let $(F, g)$ be a fixed pair in $\mathscr{C}_{\Omega}^{k+2} \times C^{k+l+1}\left(J_{\Omega}^{2}, \mathbb{R}^{q}\right)$ and let $(\tilde{F}, \tilde{g})$ be in a neighborhood of $(F, g)$. We estimate the operator norm of $C^{(j)}(F, g)-C^{(j)}(\tilde{F}, \tilde{g})$. We have

$$
\left\||| C^{(j)}(F, g)-C^{(j)}(\tilde{F}, \tilde{g})\right\|\left|\leq\left\||| C^{(j)}(F, g)-C^{(j)}(\tilde{F}, g)\right\|\|+\|\right| C^{(j)}(\tilde{F}, g-\tilde{g})\|\| .
$$

Since the map $C^{(j)}(\cdot, g)$ is continuous, it suffices to show that $\left\|\mid C^{(j)}(\tilde{F}, g-\tilde{g})\right\| \|$ goes to zero as $(\tilde{F}, \tilde{g})$ tends to $(F, g)$. So we estimate the norm $\left\|\mid C^{(j)}(\tilde{F}, g-\tilde{g})\right\| \|$ using local coordinates of $M$. Let $V_{1}, \ldots, V_{j} \in \Gamma^{k+2}\left(M, \mathbb{R}^{n}\right)$, by formula (3.11), we have

$$
\begin{gathered}
\sum_{|\alpha| \leq k} D^{\alpha}\left(\left(C^{(j)}(\tilde{F}, g-\tilde{g})\right)\left(V_{1}, \ldots, V_{j}\right)_{x}\right) \\
=\sum_{i=0}^{[k / 2]-1} \sum_{\left|\alpha_{0}+\alpha_{1}+\cdots+\alpha_{j}\right| \leq k+2-i}\left(g_{i, \alpha_{0} \cdots \alpha_{j}}\left(j_{x}^{i+2} \tilde{F}\right)-\tilde{g}_{i, \alpha_{0} \cdots \alpha_{j}}\left(j_{x}^{i+2} \tilde{F}\right)\right) \\
\cdot\left(D^{\alpha_{0}} \tilde{F}, D^{\alpha_{1}} V_{1}, \ldots, D^{\alpha_{j}} V_{j}\right),
\end{gathered}
$$

where $g_{i, \alpha_{0} \cdots \alpha_{j}}$ and $\tilde{g}_{i, \alpha_{0} \cdots \alpha_{j}}$ are $\operatorname{Hom}\left(\mathbb{R}^{n} \times \cdots \times \mathbb{R}^{n}, \mathbb{R}^{q}\right)$-valued functions which depend linearly on the derivatives of $g$ and $\tilde{g}$, respectively. We find an upper bound for the expression above in terms of the $C^{k+l+1}$-norm of the difference $g-\tilde{g}$. This will show that (3.30) goes to zero as $(\tilde{F}, \tilde{g})$ tends to $(F, g)$.

By Sobolev's lemma, a neighborhood $U$ of $F$ in $\Gamma^{k+2}\left(M, \mathbb{R}^{n}\right)$ is contained in a neighborhood $W$ of $F$ in $C^{k+2-k_{0}}\left(M, \mathbb{R}^{n}\right)$. Since the order $i+2$ of the jets $j_{x}^{i+2} \tilde{F}$ is bounded by $k+2-k_{0}$, the set $\left\{j_{x}^{i+2} \tilde{F} \in J_{\Omega}^{i+2}: x \in M, \tilde{F} \in U\right\}$ is contained in a compact set $K$ of $J_{\Omega}^{i+2}$. Therefore, the functions $g_{i, \alpha_{0} \cdots \alpha_{j}}, \tilde{g}_{i, \alpha_{0}} \cdots \alpha_{j}$ are bounded on their domain, and by using a partition of unity we can bound the difference $\left(C^{(j)}(\tilde{F}, g-\tilde{g})\right)\left(V_{1}, \ldots, V_{j}\right)$ by the $C^{k+l+1}$ norm on the compact $K$ of $g-\tilde{g}$. We get

$$
\begin{aligned}
& \left\|\left(C^{(j)}(\tilde{F}, g-\tilde{g})\right)\left(V_{1}, \ldots, V_{j}\right)\right\|_{k} \\
& \quad \leq c_{1}\|g-\tilde{g}\|_{C^{k+l+1}(K, \mathbb{R} q)}\|\tilde{F}\|_{k+2}\left\|V_{1}\right\|_{k+2} \cdots\left\|V_{j}\right\|_{k+2}
\end{aligned}
$$


where $c_{1}$ is a positive number independent of $\tilde{F} \in U$, and we used the fact that $j$ of the functions $D^{\alpha_{0}} \tilde{F}, D^{\alpha_{1}} V_{1}, \ldots, D^{\alpha_{j}} V_{j}$ are continuous. Since the subset of real numbers $\left\{\|\tilde{F}\|_{k+2} \in \mathbb{R}: \tilde{F} \in U\right\}$ is bounded, we have found that, for some $c_{2}>0$ independent of $\tilde{F} \in U,\|\| C^{(j)}(\tilde{F}, g-\tilde{g})\left\|\mid \leq c_{2}\right\| g-\tilde{g} \|_{C^{k+l+1}\left(K, \mathbb{R}^{q}\right)}$. This proves Step 3 and the lemma.

3.2. Regularity properties of $E_{d}$. In our approach to the manifold fitting problem, we compute the expected value of the distance to $F(M)$ by making a change of variables to normal coordinates. This results in an integration over $M$ and an integration over the transverse disks to $F(M)$. This double integration leads us to consider the manifold of $\Omega$-regular jets $J_{\Omega}^{p}$ in which the study of the regularity properties of $E_{d}$ can be reduced to the analysis of differentiable functions defined on $J_{\Omega}^{p}$.

Denote by $C^{k}(\Omega)$ the space of functions with continuous $k$ th-order derivatives in the interior of $\Omega$ that are bounded in $\Omega$, with norm

$$
\|f\|_{C^{k}(\Omega)}=\max _{|\alpha| \leq k} \sup \left|D^{\alpha} f\right| .
$$

For $k>m$, define the map $E_{d}: \mathscr{E}_{\Omega}^{k+2} \times C^{k+3}(\Omega) \rightarrow \mathbb{R}$ given by

$$
E_{d}(F, p)=\frac{1}{2} \int_{\Omega}\left|\mathbf{x}-F\left(\lambda_{F}(\mathbf{x})\right)\right|^{2} p(\mathbf{x}) d \mathbf{x} .
$$

Theorem 3.2. Let $k>m$. Then $E_{d}$ is a $C^{2}$ map.

Proof. By formula (2.23), the map $E_{d}$ can be written as

$$
E(F, p)=\frac{1}{2} \int_{M} \int_{\Omega_{F}}|v|^{2} p(F+v) \operatorname{det}\left(I-S_{v}\right) d v d v o l_{h} .
$$

We write this double integral in terms of a function defined on $J_{\Omega}^{2}$. For $p \in C^{k+3}(\Omega)$, consider the $C^{k+3}$ function $s_{p}: J_{\Omega}^{2} \rightarrow \mathbb{R}$ given by

$$
s_{p}\left(j_{x}^{2} F\right)=\frac{1}{2} \int_{\Omega_{F(x)}}|v|^{2} p(F(x)+v) \operatorname{det}\left(I-S_{v}\left(j_{x}^{2} F\right)\right) d v \sqrt{h\left(j_{x}^{2} F\right)} / \sqrt{g(x)},
$$

where $d v$ denotes the induced Euclidean volume element on $\Omega_{F(x)}$ and $g$ is a Riemannian metric on $M$. We have

$$
E_{d}(F, p)=\int_{M} s_{p}\left(j_{x}^{2} F\right) d v o l_{g}
$$

Therefore, in order to prove Theorem 3.2, it suffices to show that the map $\mathscr{E}_{\Omega} \times C^{k+3}(\Omega) \ni$ $(F, p) \mapsto s_{p}\left(j_{x}^{2} F\right) \in C^{\infty}(M)$ extends to a $C^{2}$ map from $\mathscr{E}_{\Omega}^{k+2} \times C^{k+3}(\Omega)$ into $\Gamma^{k}(M)$. But this follows from Lemma 3.1. 
3.3. First variation of $E_{d}$. In this section we characterize the critical embeddings of the distance functional $E_{d}$ by computing its first variational derivative at an $\Omega$-regular embedding $F$. Consider a smooth variation of $F$, that is, a smooth function

$$
\Phi: M \times(-\epsilon,+\epsilon) \longrightarrow \mathbb{R}^{n}
$$

such that $\Phi(\cdot, 0)=F$. Differentiating $\Phi$ with respect to $t$ at $t=0$ gives a vector field $V$ defined along $F(M)$. We call $V$ a variational vector field. Let $\Phi_{t}=\Phi(\cdot, t)$ and let $\lambda(\cdot, t)$ : $\Omega \rightarrow M$ be the projection index associated with $\Phi_{t}$.

Theorem 3.3. Let $F$ be an $\Omega$-regular embedding and let $\Phi$ be a smooth variation of $F$. Then

$$
\left.\frac{d E_{d}[\Phi(\cdot, t)]}{d t}\right|_{t=0}=\int_{M}\left\langle\tau_{F}, V\right\rangle d v o l_{h},
$$

where $\tau_{F}: M \rightarrow \mathbb{R}^{n}$ is the normal vector field along $F$ defined by

$$
\tau_{F}(x)=-\int_{\Omega_{F(x)}} p(F(x)+v) \operatorname{det}\left(I-S_{v}\right) v d v
$$

and $V$ is the variational vector field generated by $\Phi$. Moreover, the embedding $F$ is critical if and only if it satisfies the equation

$$
\tau_{F}=0
$$

Proof. This is a direct calculation. Set $E_{d}(t)=E_{d}[\Phi(\cdot, t)]$. Then

$$
\begin{aligned}
E_{d}^{\prime}(t) & =\frac{d}{d t} \frac{1}{2} \int_{\Omega}|\mathbf{x}-\Phi(\lambda(\mathbf{x}, t), t)|^{2} p(\mathbf{x}) d \mathbf{x} \\
& =-\int_{\Omega}\left\langle\mathbf{x}-\Phi(\lambda(\mathbf{x}, t), t), \frac{\partial \Phi(\lambda(\mathbf{x}, t), t)}{\partial x^{i}} \frac{\partial \lambda^{i}(\mathbf{x}, t)}{\partial t}+\frac{\partial \Phi(\lambda(\mathbf{x}, t), t)}{\partial t}\right\rangle p(\mathbf{x}) d \mathbf{x} .
\end{aligned}
$$

Note that for $\mathbf{x} \in \Omega$, the vector $\partial \Phi_{t} / \partial x^{i}$ is tangential to $\Phi_{t}(M)$ at $\Phi(\lambda(\mathbf{x}, t), t)$. Since $\Phi(\lambda(\mathbf{x}, t), t)$ is the point on $\Phi_{t}(M)$ nearest to $\mathbf{x}$, it follows that $\mathbf{x}-\Phi(\lambda(\mathbf{x}, t), t)$ is orthogonal to $\partial \Phi(\lambda(\mathbf{x}, t), t) / \partial x^{i}$. Thus

$$
E_{d}^{\prime}(t)=-\int_{\Omega}\left\langle\mathbf{x}-\Phi(\lambda(\mathbf{x}, t), t), \frac{\partial \Phi(\lambda(\mathbf{x}, t), t)}{\partial t}\right\rangle p(\mathbf{x}) d \mathbf{x} .
$$

By switching to normal coordinates, we obtain

$$
\begin{aligned}
E_{d}^{\prime}(0) & =-\int_{\Omega}\langle\mathbf{x}-F(\lambda(\mathbf{x}, 0)), V(\lambda(\mathbf{x}, 0))\rangle p(\mathbf{x}) d \mathbf{x} \\
& =-\int_{M} \int_{\Omega_{F(x)}}\langle v, V\rangle p(F+v) \operatorname{det}\left(I-S_{v}\right) d v d v o l_{h} .
\end{aligned}
$$

Taking the integral over the normal disks $\Omega_{F(x)}$ inside the scalar product $\langle v, V\rangle$ and writing the result in terms of $\tau_{F}$ gives (3.39). 
3.4. Second variation of $E_{d}$. We now show that the distance functional does not have local minima within the class of $\Omega$-regular embeddings. As a preparation for the statement of our result, we introduce the following notation.

For $X \in C^{\infty}\left(M, \mathbb{R}^{n}\right)$, we define the vector-valued one-form $d X \in \mathbb{R}^{n} \otimes T^{*} M$ given by the differentials $d X^{\alpha}, \alpha=1, \ldots, n$, of the components of $X$ relative to the canonical basis of $\mathbb{R}^{n}$. For a normal vector $v \in N_{F}$, we define the one-form $\langle d X, v\rangle \in T^{*} M$ by

$$
\langle d X, v\rangle(Y)=\langle d X(Y), v\rangle, \quad Y \in T M .
$$

We denote by $\langle d X, v\rangle^{\#} \in T M$ the contravariant representative of $\langle d X, v\rangle$.

Our formulation of the second variation of $E_{d}$ involves two additional quantities: the function $P_{F}: M \rightarrow \mathbb{R}$ defined by

$$
P_{F}(x)=\int_{\Omega_{F(x)}} p(F(x)+v) \operatorname{det}\left(I-S_{v}\right) d v
$$

and the map $H_{F}: C^{\infty}\left(M, \mathbb{R}^{n}\right) \times C^{\infty}\left(M, \mathbb{R}^{n}\right) \rightarrow C^{\infty}(M)$ given by

$$
\begin{aligned}
H_{F}(X, Y)=\int_{\Omega_{F}} & \left\langle\pi_{F} X-\langle d X, v\rangle^{\#},\left(I-S_{v}\right)^{-1}\left(\pi_{F} Y-\langle d Y, v\rangle^{\#}\right)\right\rangle \\
& \times p(F+v) \operatorname{det}\left(I-S_{v}\right) d v,
\end{aligned}
$$

where

$$
\pi_{F(x)}: T_{F(x)} \mathbb{R}^{n} \longrightarrow T_{x} M
$$

denotes the orthogonal projection of $T_{F(x)} \mathbb{R}^{n}$ onto $T_{x} M$. This formula may look more complicated than it really is.

Remark 3.4. In local coordinates, we have

$$
\left\langle\langle d X, v\rangle^{\#},\left(I-S_{v}\right)^{-1}\langle d Y, v\rangle^{\#}\right\rangle=\left\langle\frac{\partial X}{\partial x^{i}}, v\right\rangle h^{i j}\left[\left(I-S_{v}\right)^{-1}\right]_{j}^{k}\left\langle\frac{\partial Y}{\partial x^{k}}, v\right\rangle .
$$

We check this formula by writing $\langle d X, v\rangle^{\#}$ in local coordinates as

$$
\langle d X, v\rangle^{\#}=h^{i j}\left\langle\frac{\partial X}{\partial x^{i}}, v\right\rangle \frac{\partial F}{\partial x^{j}} .
$$

Hence

$$
\begin{aligned}
\langle\langle d X & \left., v\rangle^{\#},\left(I-S_{v}\right)^{-1}\langle d Y, v\rangle^{\#}\right\rangle \\
& =h\left(h^{i j}\left\langle\frac{\partial X}{\partial x^{i}}, v\right\rangle \frac{\partial F}{\partial x^{j}},\left(I-S_{v}\right)^{-1} h^{k l}\left\langle\frac{\partial Y}{\partial x^{k}}, v\right\rangle \frac{\partial F}{\partial x^{l}}\right) \\
& =h^{i j}\left\langle\frac{\partial X}{\partial x^{i}}, v\right\rangle h^{k l}\left\langle\frac{\partial Y}{\partial x^{k}}, v\right\rangle\left\langle\frac{\partial F}{\partial x^{j}},\left(I-S_{v}\right)^{-1} \frac{\partial F}{\partial x^{l}}\right\rangle .
\end{aligned}
$$

Define the numbers $\left[\left(I-S_{v}\right)^{-1}\right]_{l}^{m}$ by

$$
\left(I-S_{v}\right)^{-1} \frac{\partial F}{\partial x^{l}}=\left[\left(I-S_{v}\right)^{-1}\right]_{l}^{m} \frac{\partial F}{\partial x^{m}},
$$


then (3.51) gives

$$
\begin{aligned}
\langle\langle d X & \left., v\rangle^{\#},\left(I-S_{v}\right)^{-1}\langle d Y, v\rangle^{\#}\right\rangle \\
& =h^{i j}\left\langle\frac{\partial X}{\partial x^{i}}, v\right\rangle h^{k l}\left\langle\frac{\partial Y}{\partial x^{k}}, v\right\rangle\left\langle\frac{\partial F}{\partial x^{j}},\left[\left(I-S_{v}\right)^{-1}\right]_{l}^{m} \frac{\partial F}{\partial x^{m}}\right\rangle \\
& =h^{i j}\left\langle\frac{\partial X}{\partial x^{i}}, v\right\rangle h^{k l}\left\langle\frac{\partial Y}{\partial x^{k}}, v\right\rangle\left[\left(I-S_{v}\right)^{-1}\right]_{l}^{m}\left\langle\frac{\partial F}{\partial x^{j}}, \frac{\partial F}{\partial x^{m}}\right\rangle .
\end{aligned}
$$

Since $\left\langle\partial F / \partial x^{j}, \partial F / \partial x^{m}\right\rangle=h_{j m}$, a simplification of the formula above establishes (3.49). To check (3.50), we multiply both sides by a vector $Y \in T_{x} M$ and write the left-hand side in local coordinates.

Consider a smooth variation $\Phi: M \times(-\epsilon,+\epsilon) \times(-\epsilon,+\epsilon) \rightarrow \mathbb{R}^{n}$ such that $\Phi(\cdot, 0)=F$. Let $\Phi_{s t}=\Phi(\cdot, s, t)$ and let $\lambda(\cdot, s, t): \Omega \rightarrow M$ be the projection index associated with $\Phi_{s t}$.

THeOREM 3.5. Let $F$ be a critical embedding of the distance functional and let $\Phi$ be a smooth variation of $F$. Then the second variation of $E_{d}$ is given by the formula

$$
\left.\frac{\partial^{2} E_{d}[\Phi(\cdot, s, t)]}{\partial s \partial t}\right|_{(s, t)=0}=\int_{M}\left(P_{F}\langle V, W\rangle-H_{F}(V, W)\right) d v o l_{h}
$$

where $V$ and $W$ are the variational vector fields generated by $\Phi$. Moreover, all critical embeddings are saddle points of $E_{d}$.

Before proving Theorem 3.5, we discuss the meaning of the quantities $P_{F}$ and $H_{F}$. We assume that for almost every $x \in M$ the set $\operatorname{supp}(p) \cap \Omega_{F(x)}$ has positive measure as a subset of the disk $\Omega_{F(x)}$. Then, the function $P_{F}$ is positive almost everywhere since the determinant $\operatorname{det}\left(I-S_{v}\right)$ is nonvanishing in $\Omega$. Moreover, integration of $P_{F}$ over $M$ gives

$$
\int_{M} P_{F} d v o l_{h}=\int_{M} \int_{\Omega_{F(x)}} p(F(x)+v) \operatorname{det}\left(I-S_{v}\right) d v d v o l_{h}=\int_{\Omega} p(\mathbf{x}) d \mathbf{x}=1,
$$

where we switched back from normal coordinates to Cartesian coordinates. Consequently, the function $P_{F}$ is a probability density on $M$.

Proposition 3.6. The map $H_{F}$ satisfies the following properties:

(i) $H_{F}(X, Y)=H_{F}(Y, X) ; X, Y \in C^{\infty}\left(M, \mathbb{R}^{n}\right)$,

(ii) $H_{F}(X, X) \geq 0$ and $H_{F}(X, X)=0$ if and only if $X \in N_{F}$ and $\nabla^{\perp} X=0$.

Proof. Property (i) follows from the fact that the shape operator $S_{v}: T_{x} M \rightarrow T_{x} M$ is selfadjoint. To derive property (ii), observe that the operator $I-S_{v}$ is positive definite for an $\Omega$-regular embedding $F \in \mathscr{E}_{\Omega}$ since the Jacobian $\operatorname{det}\left(I-S_{v}\right)$ of the normal coordinate map does not vanish in $\Omega$. This implies that $H_{F}(X, X) \geq 0$. If $H_{F}(X, X)=0$, then

$$
\left\langle\pi_{F} X-\langle d X, v\rangle^{\#},\left(I-S_{v}\right)^{-1}\left(\pi_{F} X-\langle d X, v\rangle^{\#}\right)\right\rangle=0
$$


for all $v \in \Omega_{F(x)}$. Since $I-S_{v}$ is positive definite, we have $\pi_{F} X-\langle d X, v\rangle^{\#}=0$ for all $v \in$ $N_{F(x)}$. Set $v=0$, then $\pi_{F} X=0$, which implies that $X \in N_{F}$. On the other hand, all $(Y, v) \in$ $T_{x} M \times \Omega_{F(x)}$ is such that

$$
0=\left\langle\langle d X, v\rangle^{\#}, Y\right\rangle=\langle d X(Y), v\rangle=\left\langle v, \nabla_{Y} X\right\rangle
$$

which implies $\nabla_{Y}^{\perp} X=0$ for all $Y \in T_{x} M$. Therefore, $\nabla^{\perp} X=0$.

We conclude this section with the proof of Theorem 3.5. By (3.43),

$$
\frac{\partial^{2} E_{d}(s, t)}{\partial s \partial t}=-\frac{\partial}{\partial s} \int_{\Omega}\left\langle\mathbf{x}-\Phi(\lambda(\mathbf{x}, s, t), s, t), \frac{\partial \Phi(\lambda(\mathbf{x}, s, t), s, t)}{\partial t}\right\rangle p(\mathbf{x}) d \mathbf{x}
$$

Let

$$
g(\mathbf{x}, s, t)=-\frac{\partial}{\partial s}\left\langle\mathbf{x}-\Phi(\lambda(\mathbf{x}, s, t), s, t), \frac{\partial \Phi(\lambda(\mathbf{x}, s, t), s, t)}{\partial t}\right\rangle .
$$

Differentiation and an application of the chain rule give

$$
\begin{aligned}
g(\mathbf{x}, s, t)= & \left\langle\frac{\partial \Phi(\lambda(\mathbf{x}, s, t), s, t)}{\partial x^{i}} \frac{\partial \lambda^{i}(\mathbf{x}, s, t)}{\partial s}, \frac{\partial \Phi(\lambda(\mathbf{x}, s, t), s, t)}{\partial t}\right\rangle \\
& +\left\langle\frac{\partial \Phi(\lambda(\mathbf{x}, s, t), s, t)}{\partial s}, \frac{\partial \Phi(\lambda(\mathbf{x}, s, t), s, t)}{\partial t}\right\rangle \\
& -\left\langle\mathbf{x}-\Phi(\lambda(\mathbf{x}, s, t), s, t), \frac{\partial^{2} \Phi(\lambda(\mathbf{x}, s, t), s, t)}{\partial x^{i} \partial t} \frac{\partial \lambda^{i}(\mathbf{x}, s, t)}{\partial s}\right\rangle \\
& -\left\langle\mathbf{x}-\Phi(\lambda(\mathbf{x}, s, t), s, t), \frac{\partial^{2} \Phi(\lambda(\mathbf{x}, s, t), s, t)}{\partial s \partial t}\right\rangle
\end{aligned}
$$

We compute $g(\mathbf{x}, 0)$. Let $Z=\partial^{2} \Phi(\cdot, 0) / \partial s \partial t$. Substituting the identities

$$
\begin{aligned}
\frac{\partial \Phi(\lambda(\mathbf{x}, 0), 0)}{\partial x^{i}} & =\frac{\partial F}{\partial x^{i}}(\lambda(\mathbf{x}, 0)) \\
\frac{\partial \Phi(\lambda(\mathbf{x}, 0), 0)}{\partial s} & =V(\lambda(\mathbf{x}, 0)) \\
\frac{\partial \Phi(\lambda(\mathbf{x}, 0), 0)}{\partial t} & =W(\lambda(\mathbf{x}, 0)) \\
\frac{\partial \Phi(\lambda(\mathbf{x}, 0), 0)}{\partial x^{j}} \frac{\partial \lambda^{j}(\mathbf{x}, 0)}{\partial s} & =\frac{\partial \lambda}{\partial s}(\mathbf{x}, 0), \\
\frac{\partial^{2} \Phi(\lambda(\mathbf{x}, 0), 0)}{\partial x^{j} \partial t} \frac{\partial \lambda^{j}(\mathbf{x}, 0)}{\partial s} & =d W(\lambda(\mathbf{x}, 0))\left(\frac{\partial \lambda}{\partial s}(\mathbf{x}, 0)\right)
\end{aligned}
$$


into (3.60) at $s, t=0$ gives

$$
\begin{aligned}
g(\mathbf{x}, 0)= & \left\langle\frac{\partial \lambda}{\partial s}(\mathbf{x}, 0)+V(\lambda(\mathbf{x}, 0)), W(\lambda(\mathbf{x}, 0))\right\rangle \\
& -\left\langle\mathbf{x}-F(\lambda(\mathbf{x}, 0)), d W(\lambda(\mathbf{x}, 0))\left(\frac{\partial \lambda}{\partial s}(\mathbf{x}, 0)\right)+Z(\lambda(\mathbf{x}, 0))\right\rangle .
\end{aligned}
$$

Since $\partial \lambda(\mathbf{x}, 0) / \partial s$ is tangent to $M$, we can write

$$
\left\langle\frac{\partial \lambda}{\partial s}(\mathbf{x}, 0), W(\lambda(\mathbf{x}, 0))\right\rangle=\left\langle\frac{\partial \lambda}{\partial s}(\mathbf{x}, 0), \pi_{F(\lambda(\mathbf{x}, 0))} W(\lambda(\mathbf{x}, 0))\right\rangle
$$

Substituting (3.67) into (3.66) and collecting the factors of $\partial \lambda(\mathbf{x}, 0) / \partial s$ gives

$$
\begin{aligned}
g(\mathbf{x}, 0)= & \langle V(\lambda(\mathbf{x}, 0)), W(\lambda(\mathbf{x}, 0))\rangle \\
& +\left\langle\pi_{F(\lambda(\mathbf{x}, 0))} W(\lambda(\mathbf{x}, 0))-\langle d W(\lambda(\mathbf{x}, 0)), \mathbf{x}-F(\lambda(\mathbf{x}, 0))\rangle^{\#}, \frac{\partial \lambda}{\partial s}(\mathbf{x}, 0)\right\rangle \\
& -\langle\mathbf{x}-F(\lambda(\mathbf{x}, 0)), Z(\lambda(\mathbf{x}, 0))\rangle .
\end{aligned}
$$

We need a formula for $\partial \lambda(\mathbf{x}, 0) / \partial s$. Observe that

$$
\left\langle\mathbf{x}-\Phi(\lambda(\mathbf{x}, s, t), s, t), \frac{\partial \Phi(\lambda(\mathbf{x}, s, t), s, t)}{\partial x^{i}}\right\rangle=0, \quad i=1, \ldots, m
$$

identically in $s$ and $t$ since $\mathbf{x}-\Phi(\lambda(\mathbf{x}, s, t), s, t)$ is normal to $\Phi_{s t}(M)$ and $\partial \Phi_{s t} / \partial x^{i}$ is tangential to $\Phi_{s t}(M)$. Differentiating with respect to $s$ at $s=0$ gives

$$
\begin{aligned}
-\left\langle\frac{\partial \Phi(\lambda(\mathbf{x}, 0), 0)}{\partial x^{j}} \frac{\partial \lambda^{j}(\mathbf{x}, 0)}{\partial s}+\frac{\partial \Phi(\lambda(\mathbf{x}, 0), 0)}{\partial s}, \frac{\partial \Phi(\lambda(\mathbf{x}, 0), 0)}{\partial x^{i}}\right\rangle \\
+\left\langle\mathbf{x}-\Phi(\lambda(\mathbf{x}, 0), 0), \frac{\partial^{2} \Phi(\lambda(\mathbf{x}, 0), 0)}{\partial x^{i} \partial x^{j}} \frac{\partial \lambda^{j}(\mathbf{x}, 0)}{\partial s}+\frac{\partial^{2} \Phi(\lambda(\mathbf{x}, 0), 0)}{\partial x^{i} \partial s}\right\rangle=0
\end{aligned}
$$

Using the identities (3.61) through (3.65) gives

$$
\begin{aligned}
-\left\langle\frac{\partial \lambda}{\partial s}(\mathbf{x}, 0)+V(\lambda(\mathbf{x}, 0)), \frac{\partial F}{\partial x^{i}}(\lambda(\mathbf{x}, 0))\right\rangle & \\
& +\left\langle\mathbf{x}-F(\lambda(\mathbf{x}, 0)), \nabla(\partial \lambda / \partial s)(\mathbf{x}, 0) \frac{\partial F}{\partial x^{i}}(\lambda(\mathbf{x}, 0))+d V(\lambda(\mathbf{x}, 0))\left(\frac{\partial F}{\partial x^{i}}(\lambda(\mathbf{x}, 0))\right)\right\rangle=0 .
\end{aligned}
$$

We use (2.5) to write

$$
\left\langle\mathbf{x}-F(\lambda(\mathbf{x}, 0)), \nabla_{(\partial \lambda / \partial s)(\mathbf{x}, 0)} \frac{\partial F}{\partial x^{i}}(\lambda(\mathbf{x}, 0))\right\rangle=\left\langle S_{\mathbf{x}-F(\lambda(\mathbf{x}, 0))} \frac{\partial \lambda}{\partial s}(\mathbf{x}, 0), \frac{\partial F}{\partial x^{i}}(\lambda(\mathbf{x}, 0))\right\rangle .
$$


Substituting (3.72) into (3.71) and collecting the factors of $\partial \lambda / \partial s$ and $\partial F / \partial x^{i}$ gives

$$
\begin{aligned}
& \left\langle\left(S_{\mathbf{x}-F(\lambda(\mathbf{x}, 0))}-I\right) \frac{\partial \lambda}{\partial s}(\mathbf{x}, 0)+\langle d V(\lambda(\mathbf{x}, 0)), \mathbf{x}-F(\lambda(\mathbf{x}, 0))\rangle^{\#}\right. \\
& \left.\quad-\pi_{F(\lambda(\mathbf{x}, 0))} V(\lambda(\mathbf{x}, 0)), \frac{\partial F}{\partial x^{i}}(\lambda(\mathbf{x}, 0))\right\rangle=0 .
\end{aligned}
$$

This holds for every basis element $\partial F(\lambda(\mathbf{x}, 0)) / \partial x^{i}$ in the local coordinate frame. Therefore, we must have

$$
\left(S_{\mathbf{x}-F(\lambda(\mathbf{x}, 0))}-I\right) \frac{\partial \lambda}{\partial s}(\mathbf{x}, 0)+\langle d V(\lambda(\mathbf{x}, 0)), \mathbf{x}-F(\lambda(\mathbf{x}, 0))\rangle^{\#}-\pi_{F(\lambda(\mathbf{x}, 0))} V(\lambda(\mathbf{x}, 0))=0 .
$$

Since $F \in \mathscr{E}_{\Omega}$, the operator $S_{\mathbf{x}-F(\lambda(\mathbf{x}, 0))}-I: T_{\lambda(\mathbf{x}, 0)} M \rightarrow T_{\lambda(\mathbf{x}, 0)} M$ is nonsingular for all $\mathbf{x} \in \Omega$. Solving for $\partial \lambda(\mathbf{x}, 0) / \partial s$ in (3.74) gives

$$
\frac{\partial \lambda}{\partial s}(\mathbf{x}, 0)=\left(I-S_{\mathbf{x}-F(\lambda(\mathbf{x}, 0))}\right)^{-1}\left(\langle d V(\lambda(\mathbf{x}, 0)), \mathbf{x}-F(\lambda(\mathbf{x}, 0))\rangle^{\#}-\pi_{F(\lambda(\mathbf{x}, 0))} V(\lambda(\mathbf{x}, 0))\right) .
$$

Substituting into (3.68) gives

$$
\begin{aligned}
g(\mathbf{x}, 0)=\langle & V(\lambda(\mathbf{x}, 0)), W(\lambda(\mathbf{x}, 0))\rangle \\
- & \left\langle\pi_{F(\lambda(\mathbf{x}, 0))} V(\lambda(\mathbf{x}, 0))-\langle d V(\lambda(\mathbf{x}, 0)), \mathbf{x}-F(\lambda(\mathbf{x}, 0))\rangle^{\#},\right. \\
& \left.\left(I-S_{\mathbf{x}-F(\lambda(\mathbf{x}, 0))}\right)^{-1}\left(\pi_{F(\lambda(\mathbf{x}, 0))} W(\lambda(\mathbf{x}, 0))-\langle d W(\lambda(\mathbf{x}, 0)), \mathbf{x}-F(\lambda(\mathbf{x}, 0))\rangle^{\#}\right)\right\rangle \\
- & \langle\mathbf{x}-F(\lambda(\mathbf{x}, 0)), Z(\lambda(\mathbf{x}, 0))\rangle .
\end{aligned}
$$

Multiplying by the probability distribution $p(\mathbf{x})$, integrating over $\Omega$, and reverting to normal coordinates gives

$$
\begin{aligned}
\left.\frac{\partial^{2} E_{d}}{\partial s \partial t}\right|_{(s, t)=0}= & \int_{\Omega} g(\mathbf{x}, 0) p(\mathbf{x}) d \mathbf{x} \\
= & \int_{M} \int_{\Omega_{F}}\left\{\langle V, W\rangle-\left\langle\pi_{F} V-\langle d V, v\rangle^{\#},\left(I-S_{v}\right)^{-1}\left(\pi_{F} W-\langle d W, v\rangle^{\#}\right)\right\rangle\right. \\
& \quad-\langle v, Z\rangle\} p(F+v) \operatorname{det}\left(I-S_{v}\right) d v d v o l_{h} .
\end{aligned}
$$

Distributing the factor $p(F+v) \operatorname{det}\left(I-S_{v}\right)$ and writing the result in terms of $P_{F}, H_{F}$, and $\tau_{F}$ gives

$$
\left.\frac{\partial^{2} E_{d}}{\partial s \partial t}\right|_{(s, t)=0}=\int_{M}\left\{P_{F}\langle V, W\rangle-H_{F}(V, W)+\left\langle\tau_{F}, Z\right\rangle\right\} d v o l_{h} .
$$

If $F$ is a critical embedding of $E_{d}$, then $\tau_{F}=0$ and formula (3.54) follows. 
To prove that critical embeddings are saddle points of $E_{d}$, set $W=V$ with $V \in N_{F}$ in (3.54). If $\nabla^{\perp} V=0$, by Proposition 3.6, we have $H_{F}(V, V)=0$. This gives a positive second derivative.

On the other hand, since $V \in N_{F}, \pi_{F} V=0$. Hence

$$
H_{F}(V, V)=\int_{\Omega_{F}}\left\langle\langle d V, v\rangle^{\#},\left(I-S_{v}\right)^{-1}\langle d V, v\rangle^{\#}\right\rangle p(F+v) \operatorname{det}\left(I-S_{v}\right) d v .
$$

For an operator $A: T_{x} M \rightarrow T_{x} M$, let $|A|$ denote its operator norm. We can estimate $H_{F}(V, V)$ as

$$
H_{F}(V, V) \leq \int_{\Omega_{F}}\left|\left(I-S_{v}\right)^{-1}\right|\left|\langle d V, v\rangle^{\#}\right|^{2} p(F+v) \operatorname{det}\left(I-S_{v}\right) d v .
$$

Now we give an estimate of the squared norm of the vector $\langle d V, v\rangle^{\#} \in T M$. Consider a local orthonormal frame $\left\{e_{i}\right\}$ along $F(M)$. We have

$$
\left|\langle d V, v\rangle^{\#}\right|^{2}=\sum_{i=1}^{m}\left\langle e_{i},\langle d V, v\rangle^{\#}\right\rangle^{2} .
$$

Since, by definition, $\left\langle e_{i},\langle d V, v\rangle^{\#}\right\rangle=\left\langle\nabla_{e_{i}} V, v\right\rangle$ and since $v \in N_{F(x)}$ is a normal vector to $F(M)$, we have

$$
\left|\langle d V, v\rangle^{\#}\right|^{2}=\sum_{i=1}^{m}\left\langle\nabla_{e_{i}}^{\perp} V, v\right\rangle^{2} \leq \sum_{i=1}^{m}\left|\nabla_{e_{i}}^{\perp} V\right|^{2}|v|^{2}
$$

by the Schwartz inequality. Substituting into (3.80) and factoring out the squared norm $\left|\nabla^{\perp} V\right|^{2} \equiv \sum_{i=1}^{m}\left|\nabla_{e_{i}}^{\perp} V\right|^{2}$ gives

$$
H_{F}(V, V) \leq\left|\nabla^{\perp} V\right|^{2} \int_{\Omega_{F}}\left|\left(I-S_{v}\right)^{-1}\right||v|^{2} p(F+v) \operatorname{det}\left(I-S_{v}\right) d v .
$$

Therefore, choosing a variation with large $\left|\nabla^{\perp} V\right|$ and small $|V|$ (in the $L^{2}$ sense) in (3.54) leads to a negative second derivative.

\section{Extremal properties of the energy functional}

In this section we lay the ground for the study of the general fitting problem. Our main concerns are the variational properties of $E$ on the class of $\Omega$-regular embeddings. We characterize the critical points of $E$ in terms of harmonic maps and the extrinsic geometry of the embedding $F: M \rightarrow \mathbb{R}^{n}$. We also investigate conditions under which a critical embedding yields a local minimum.

4.1. Regularity properties of $E$. In this subsection we continue with the analysis of the regularity of $E$ started in Section 3.2. We will show that the harmonic functional $E_{h}$ is a $C^{\infty}$ map defined on an appropriate Sobolev space.

For $k>m$, consider the map $E: \mathscr{C}_{\Omega}^{k+2} \times C^{k+3}(\Omega) \times \mathbb{R} \rightarrow \mathbb{R}$ given by

$$
E(F, p, k)=E_{d}(F, p)+k E_{h}(F)
$$


where $E_{d}$ is defined by (3.34) and $E_{h}$ is the harmonic functional (1.2). Our goal in this section is to prove the following theorem.

Theorem 4.1. Let $k>m$. Then $E$ is a $C^{2}$ map.

Proof. We show that $E_{h}: \Gamma^{k+2}\left(M, \mathbb{R}^{n}\right) \rightarrow \mathbb{R}$ is a smooth map. Then, from Theorem 3.2, it will follow that $E$ is $C^{2}$.

Consider a local orthonormal frame $\left\{e_{i}^{M}\right\} \subset T M$ with respect to the metric $g$. Let $F, V \in \Gamma^{k+2}\left(M, \mathbb{R}^{n}\right)$. We have

$$
\begin{aligned}
\left|\frac{E_{h}(F+t V)-E_{h}(F)}{t}-\int_{M} \delta^{i j}\left\langle d F\left(e_{i}^{M}\right), d V\left(e_{j}^{M}\right)\right\rangle d v o l_{g}\right| & \mid \frac{(1 / 2) \int_{M} \delta^{i j}\left\{\left\langle d(F+t V)\left(e_{i}^{M}\right), d(F+t V)\left(e_{j}^{M}\right)\right\rangle-\left\langle d F\left(e_{i}^{M}\right), d F\left(e_{j}^{M}\right)\right\rangle\right\} d v o l_{g}}{t} \\
\quad & -\int_{M} \delta^{i j}\left\langle d F\left(e_{i}^{M}\right), d V\left(e_{j}^{M}\right)\right\rangle d v o l_{g} \mid \\
= & \frac{t}{2} \int_{M} \delta^{i j}\left\langle d V\left(e_{i}^{M}\right), d V\left(e_{j}^{M}\right)\right\rangle d v o l_{g} .
\end{aligned}
$$

Thus

$$
\lim _{t \rightarrow 0}\left|\frac{E_{h}(F+t V)-E_{h}(F)}{t}-\int_{M} \delta^{i j}\left\langle d F\left(e_{i}^{M}\right), d V\left(e_{j}^{M}\right)\right\rangle d v o l_{g}\right|=0
$$

Then, the Gâteaux derivative $\delta E_{h}(F) \in \Gamma^{k+2}\left(M, \mathbb{R}^{n}\right)^{*}$ of $E_{h}$ at $F$ is given by

$$
\delta E_{h}(F) \cdot V=\int_{M} \delta^{i j}\left\langle d F\left(e_{i}^{M}\right), d V\left(e_{j}^{M}\right)\right\rangle d v o l_{g}
$$

We now prove that this is continuous as a map $\delta E_{h}: \Gamma^{k+2}\left(M, \mathbb{R}^{n}\right) \rightarrow \Gamma^{k+2}\left(M, \mathbb{R}^{n}\right)^{*}$. This will imply that $E_{h}$ is a $C^{1}$ map [11, page 37]. For $F_{1}, F_{2} \in \Gamma^{k+2}\left(M, \mathbb{R}^{n}\right)$, we have

$$
\begin{aligned}
& \left|\delta E_{h}\left(F_{1}\right) \cdot V-\delta E_{h}\left(F_{2}\right) \cdot V\right| \\
& \quad=\left|\int_{M} \delta^{i j}\left\langle d F_{1}\left(e_{i}^{M}\right), d V\left(e_{j}^{M}\right)\right\rangle d v o l_{g}-\int_{M} \delta^{i j}\left\langle d F_{2}\left(e_{i}^{M}\right), d V\left(e_{j}^{M}\right)\right\rangle d v o l_{g}\right| \\
& \quad \leq\left\|F_{1}-F_{2}\right\|_{1}\|V\|_{1} .
\end{aligned}
$$

Thus $\left\|\delta E_{h}\left(F_{1}\right)-\delta E_{h}\left(F_{2}\right)\right\|_{\Gamma^{k+2}\left(M, \mathbb{R}^{n}\right) *} \leq\left\|F_{1}-F_{2}\right\|_{k+2}$; that is, $E_{h}$ is a $C^{1}$ map. By (4.4), the map $\delta E_{h}$ is linear, therefore smooth. This proves that $E_{h}$ is smooth. 
4.2. First variation of $E$. In this subsection we characterize the critical embeddings of the functional $E$ by computing its first variational derivative at an $\Omega$-regular embedding $F$.

Let $\Delta_{g}$ denote the Laplace-Beltrami operator relative to the metric $g$ in $M$. In local coordinates,

$$
\Delta_{g} f=-\frac{1}{\sqrt{g}} \frac{\partial}{\partial x^{i}}\left(\sqrt{g} g^{i j} \frac{\partial f}{\partial x^{j}}\right) .
$$

Consider a local orthonormal frame $\left\{e_{i}^{M}\right\} \subset T M$ with respect to the metric $g$ and let $h$ be the Euclidean metric induced on $M$.

Theorem 4.2. Let $F$ be an $\Omega$-regular embedding and let $\Phi$ be a smooth variation of $F$. Then

$$
\left.\frac{d E[\Phi(\cdot, t)]}{d t}\right|_{t=0}=\int_{M}\left\langle\tau_{F}, V\right\rangle d \operatorname{vol}_{h}+k \int_{M}\left\langle\Delta_{g} F, V\right\rangle d v o l_{g}
$$

where $V$ is the variational vector field generated by $\Phi$. Moreover, the embedding $F$ is critical if and only if $F: M \rightarrow F(M)$ is a harmonic map and satisfies the equation

$$
\tau_{F} \frac{\sqrt{h}}{\sqrt{g}}-k \delta^{i j} B\left(e_{i}^{M}, e_{j}^{M}\right)=0,
$$

where $\tau_{F}$ is the normal vector field to $F$ defined by (3.40).

We call (4.8) the transversal Euler-Lagrange equation of a critical embedding. Before proving Theorem 4.2, we discuss some examples.

Example 4.3. Curves in $\mathbb{R}^{n}$. Let $M=S^{1}$. Then a critical embedding $F$ has a constant speed parameterization and for all $X \in \mathbb{R}^{n}$,

$$
-\Lambda_{1}(X)+\Lambda_{2}\left(\frac{d \mathbf{T}}{d s}, X\right)-\left(\frac{k L_{F}}{2 \pi}\right)\left\langle\frac{d \mathbf{T}}{d s}, X\right\rangle=0,
$$

where $L_{F}$ denotes the length of $F\left(S^{1}\right), \mathbf{T} \in C^{\infty}\left(S^{1}, \mathbb{R}^{n}\right)$ is the unit tangent along $F\left(S^{1}\right)$, and $d \mathbf{T} / d s \in C^{\infty}\left(S^{1}, \mathbb{R}^{n}\right)$ denotes its derivative with respect to arc length. To verify (4.9) we compute the Euler-Lagrange equation of a critical curve. We use the identities

$$
\frac{\sqrt{h}}{\sqrt{g}}=\left|F^{\prime}\right|, \quad \Delta_{g} F=-F^{\prime \prime} .
$$

By Example 2.4, we have $\operatorname{det}\left(I-S_{v}\right)=1-\langle d \mathbf{T} / d s, v\rangle$. Let $X \in \mathbb{R}^{n}$. By (3.40),

$$
\left\langle\tau_{F}, X\right\rangle=\left\langle-\int_{\Omega_{F}} p(F+v)\left(1-\left\langle\frac{d \mathbf{T}}{d s}, v\right\rangle\right) v d v, X\right\rangle
$$

We expand this out and write the result in terms of the transverse moments $\Lambda_{k}$. We get

$$
\left\langle\tau_{F}, X\right\rangle=-\Lambda_{1}(X)+\Lambda_{2}\left(\frac{d \mathbf{T}}{d s}, X\right)
$$


By the definition of arc length,

$$
s=\int_{0}^{u}\left|F^{\prime}\right| d u
$$

we can write $d \mathbf{T} / d s$ as $\mathbf{T}^{\prime} /\left|F^{\prime}\right|$ in (4.12). We get

$$
\left\langle\tau_{F}, X\right\rangle=-\Lambda_{1}(X)+\Lambda_{2}\left(\frac{\mathrm{T}^{\prime}}{\left|F^{\prime}\right|}, X\right) .
$$

We scalar-multiply (4.8) by $X$ and use (4.10) to write the result

$$
\left\langle\tau_{F}, X\right\rangle\left|F^{\prime}\right|-k\left\langle F^{\prime \prime}, X\right\rangle=0 .
$$

By (4.14), we get

$$
\left(-\Lambda_{1}(X)+\Lambda_{2}\left(\frac{\mathbf{T}^{\prime}}{\left|F^{\prime}\right|}, X\right)\right)\left|F^{\prime}\right|-k\left\langle F^{\prime \prime}, X\right\rangle=0 .
$$

Set $X=F^{\prime}$. Since $\Lambda_{k}\left(F^{\prime}\right)=0$, we get $\left\langle F^{\prime \prime}, F^{\prime}\right\rangle=0$. Thus, a critical embedding $F$ has constant speed $\left|F^{\prime}\right|$ which can be computed by using (4.13) as

$$
\left|F^{\prime}\right|=\frac{L_{F}}{2} \pi
$$

The second derivative $F^{\prime \prime}$ can be written as $F^{\prime \prime}=\left(\left|F^{\prime}\right| \mathbf{T}\right)^{\prime}=\left|F^{\prime}\right| \mathbf{T}^{\prime}$. Substituting into (4.16) and taking the derivatives with respect to arc length gives

$$
\left(-\Lambda_{1}(X)+\Lambda_{2}\left(\frac{d \mathbf{T}}{d s}, X\right)\right)\left|F^{\prime}\right|-k\left\langle\frac{\left|F^{\prime}\right|^{2} d \mathbf{T}}{d s}, X\right\rangle=0 .
$$

Substituting (4.17) into (4.18) and dividing the result by $\left|F^{\prime}\right|$ gives (4.9).

Example 4.4. For radial distributions relative to a curve $\Gamma$ (see Figure 4.1), the covariance tensor is a constant multiple $\left|\sigma_{\perp}^{2}\right|$ of the Euclidean scalar product restricted to $N_{F(u)}$ (Example 2.8). Let $F: S^{1} \rightarrow \mathbb{R}^{n}$ be a constant speed parameterization of $\Gamma$ and set $k=$ $2 \pi\left|\sigma_{\perp}^{2}\right| / L_{F}^{2}$. For this value of $k$ we have that $\Gamma$ is a critical curve. To check this, we verify (4.9) for all $X \in \mathbb{R}^{n}$. By Example 2.8, we have $\mu_{\perp}=0$, which implies $\Lambda_{1}=0$. Using Definition 2.7 to write $\Lambda_{2}$ in terms of $\sigma_{\perp}^{2}$ gives

$$
-\Lambda_{1}(X)+\Lambda_{2}\left(\frac{d \mathbf{T}}{d s}, X\right)-\frac{k L_{F}}{2 \pi}\left\langle\frac{d \mathbf{T}}{d s}, X\right\rangle=\Lambda_{0} \sigma_{\perp}^{2}\left(\frac{d \mathbf{T}}{d s}, X\right)-\frac{k L_{F}}{2 \pi}\left\langle\frac{d \mathbf{T}}{d s}, X\right\rangle .
$$

Since $\sigma_{\perp}^{2}$ is a constant multiple $\left|\sigma_{\perp}^{2}\right|$ of the Euclidean scalar product restricted to $N_{F(u)}$ and since $d \mathbf{T} / d s \in N_{F(s)}$, we have

$$
\Lambda_{0} \sigma_{\perp}^{2}\left(\frac{d \mathbf{T}}{d s}, X\right)-\frac{k L_{F}}{2 \pi}\left\langle\frac{d \mathbf{T}}{d s}, X\right\rangle=\Lambda_{0}\left|\sigma_{\perp}^{2}\right|\left\langle\frac{d \mathbf{T}}{d s}, X\right\rangle-\left(\frac{k L_{F}}{2} \pi\right)\left\langle\frac{d \mathbf{T}}{d s}, X\right\rangle
$$




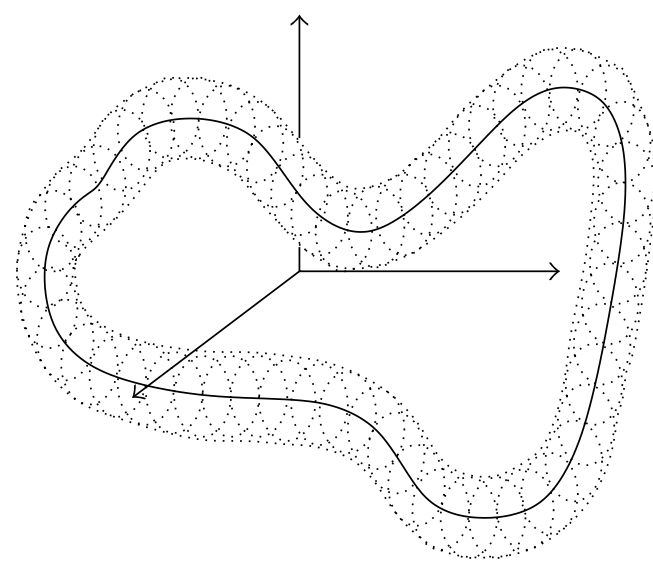

Figure 4.1. A radial distribution relative to a curve.

We now compute an explicit formula for $\Lambda_{0}$. We switch to normal coordinates in the integral $\int_{\Omega} p(\mathbf{x}) d \mathbf{x}$ and express the result in terms of the transverse moments,

$$
\begin{aligned}
1 & =\int_{\Omega} p(\mathbf{x}) d \mathbf{x}=\int_{0}^{L_{F}} \int_{\Omega_{F(s)}} p(F(s)+v)\left(1-\left\langle v, \frac{d \mathbf{T}}{d s}\right\rangle\right) d v d s \\
& =\int_{0}^{L_{F}}\left(\Lambda_{0}-\Lambda_{1}\left(\frac{d \mathbf{T}}{d s}\right)\right) d s .
\end{aligned}
$$

Since $\Lambda_{0}$ is constant and $\Lambda_{1}=0$, we have that $\Lambda_{0}=1 / L_{F}$. Substituting into (4.19) gives

$$
\Lambda_{0} \sigma_{\perp}^{2}\left(\frac{d \mathbf{T}}{d s}, X\right)-\frac{k L_{F}}{2 \pi}\left\langle\frac{d \mathbf{T}}{d s}, X\right\rangle=\left(\frac{1}{L_{F}}\right)\left|\sigma_{\perp}^{2}\right|\left\langle\frac{d \mathbf{T}}{d s}, X\right\rangle-\left(\frac{k L_{F}}{2} \pi\right)\left\langle\frac{d \mathbf{T}}{d s}, X\right\rangle .
$$

Since $k=2 \pi\left|\sigma_{\perp}^{2}\right| / L_{F}^{2}$, we have that (4.19) vanishes identically. This shows that $\Gamma$ is a critical curve.

Example 4.5 (the annulus). In this example we consider a special probability distribution in the plane. Let

$$
\Omega=\left\{\mathbf{x} \in \mathbb{R}^{2}: r_{1} \leq|\mathbf{x}| \leq r_{2}\right\} .
$$

Assume that $p$ depends only on $|\mathbf{x}|$. Let

$$
\nu^{k}=\int_{r_{1}}^{r_{2}} \rho^{k} p(\rho) d \rho
$$

We assert that if the spring constant $k$ satisfies

$$
r_{1}<\frac{1 / 2 \pi-v^{2}}{k}<r_{2}
$$

then the circle of radius $r=\left(1 / 2 \pi-v^{2}\right) / k$ is a critical curve. 
To prove the assertion we verify (4.8). We consider the embedding

$$
F(u)=r(\cos (u), \sin (u)), \quad u \in[0,2 \pi]
$$

Denote by $\mathbf{N}$ the unit normal vector field along $F\left(S^{1}\right)$ oriented so that the pair $(\mathbf{T}, \mathbf{N})$ is consistent with the standard orientation of $\mathbb{R}^{2}$. We have the identities

$$
\left\langle\frac{\mathbf{T}^{\prime}}{\left|F^{\prime}\right|}, \mathbf{N}\right\rangle=-\frac{1}{r}, \quad \frac{\sqrt{h}}{\sqrt{g}}=r, \quad F^{\prime \prime}=-r \mathbf{N} .
$$

To compute $\tau_{F}$, we note that for this type of distribution the disks $\Omega_{F(u)}$ are line segments perpendicular to the circle $F\left(S^{1}\right)$ whose length is equal to the width of the annulus, that is, $r_{2}-r_{1}$. Then the transverse integration along $\Omega_{F(u)}$ in the definition of $\tau_{F}$ can be performed by computing an ordinary integral on the interval $\left[r_{1}-r, r_{2}-r\right]$. We use the first identity in (4.27) to write $\tau_{F}$ as

$$
\begin{aligned}
\tau_{F} & =-\int_{\Omega_{F}} p(F+v)\left(1-\left\langle\frac{\mathbf{T}^{\prime}}{\left|F^{\prime}\right|}, v \mathbf{N}\right\rangle\right) v \mathbf{N} d v \\
& =-\int_{r_{1}-r}^{r_{2}-r} p(r+v)\left(1+\frac{v}{r}\right) v \mathbf{N} d v .
\end{aligned}
$$

Simplifying and making the change of variables $\rho=r+v$ gives

$$
\tau_{F}=-\frac{1}{r} \int_{r_{1}}^{r_{2}} \rho(\rho-r) p(\rho) d \rho \mathbf{N}
$$

which, written in terms of the $\nu^{k}$ 's, reads

$$
\tau_{F}=-\frac{\nu^{2}+\nu^{1}}{r} \mathbf{N}
$$

We give an explicit formula for $\nu^{1}$. By changing to polar coordinates in the integral $\int_{\Omega} p(\mathbf{x}) d \mathbf{x}$, we get

$$
1=\int_{\Omega} p(\mathbf{x}) d \mathbf{x}=\int_{0}^{2 \pi} \int_{r_{1}}^{r_{2}} p(\rho) \rho d \rho d \theta,
$$

from which it follows that $1=2 \pi \nu^{1}$ or $\nu^{1}=1 / 2 \pi$. Substituting this into (4.30) gives

$$
\tau_{F}=\frac{-v^{2}+1 / 2 \pi}{r} \mathbf{N}
$$

We now write (4.8) multiplying $\tau_{F}$ by $\sqrt{h} / \sqrt{g}=r$ and adding $k \Delta_{g} F=-k r \mathbf{N}$ :

$$
\tau_{F} \frac{\sqrt{h}}{\sqrt{g}}+k \Delta_{g} F=\left(-v^{2}+\frac{1}{2} \pi\right) \mathbf{N}-k r \mathbf{N} .
$$

This expression vanishes identically since $r=\left(1 / 2 \pi-v^{2}\right) / k$. This shows that $F$ is a critical embedding. 
Example 4.6. The spherical shell. We now consider a probability distribution that is supported on the region

$$
\Omega=\left\{\mathbf{x} \in \mathbb{R}^{3}: r_{1} \leq|\mathbf{x}| \leq r_{2}\right\} .
$$

We assume that $p$ depends only on $|\mathbf{x}|$. Let

$$
v^{k}=\int_{r_{1}}^{r_{2}} \rho^{k} p(\rho) d \rho
$$

We assert that if the spring constant $k$ satisfies

$$
r_{1}<\frac{v^{3}}{2 k+1 / 4 \pi}<r_{2}
$$

then the sphere $S_{r}^{2}$ of radius $r=\nu^{3} /(2 k+1 / 4 \pi)$ is critically embedded in $\Omega$.

To verify this assertion we check that the inclusion $i: S^{2} \hookrightarrow \mathbb{R}^{3}$ satisfies the EulerLagrange equation (4.8). For this, we compute the quantities appearing in it. First notice that the quotient of volume elements is given by

$$
\frac{\sqrt{h}}{\sqrt{g}}=r^{2},
$$

since the sphere $S_{r}^{2}$ is a dilation by a factor of $r$ of the standard unit sphere $S^{2}$. Let $\hat{\mathbf{n}} \in$ $C^{\infty}\left(M, \mathbb{R}^{n}\right)$ be the outer unit normal vector field along $S_{r}^{2}$, and denote by $v \hat{\mathbf{n}} \in N_{F(x)}$, $v \in \mathbb{R}$, any vector normal to $S_{r}^{2}$ at $F(x)$. By the symmetry of the sphere, the shape operator $S$ is a scalar multiplication by $-1 / r$. Then, we can write a formula for $\tau_{F}$ as

$$
\tau_{F}=-\int_{\Omega_{F}} p(F+v \hat{\mathbf{n}}) \operatorname{det}\left(I-S_{v}\right) v \hat{\mathbf{n}} d v=-\int_{r_{1}-r}^{r_{2}-r} p(r+v)\left(1+\frac{v}{r}\right)^{2} v \hat{\mathbf{n}} d v .
$$

To evaluate this integral, we make the change of variables $\rho=r+v$ and write the result in terms of the $v^{i}$ 's. We get

$$
\tau_{F}=\frac{-\left(\nu^{3}-r \nu^{2}\right) \hat{\mathbf{n}}}{r^{2}}
$$

We next compute an explicit formula for $v^{2}$ using the identity $\int_{\Omega} p(\mathbf{x}) d \mathbf{x}=1$ :

$$
1=\int_{\Omega} p(\mathbf{x}) d \mathbf{x}=\operatorname{Vol}\left(S^{2}\right) \int_{r_{1}}^{r_{2}} p(\rho) \rho^{2} d \rho=4 \pi v^{2}
$$

Substituting $\nu^{2}=1 / 4 \pi$ into (4.39) gives

$$
\tau_{F}=\frac{-\left(\nu^{3}-r / 4 \pi\right) \hat{\mathbf{n}}}{r^{2}} .
$$

Finally, we write a formula for $\delta^{i j} B\left(e_{i}^{M}, e_{j}^{M}\right)$ in terms of the shape operator $S$ as

$$
\delta^{i j} B\left(e_{i}^{M}, e_{j}^{M}\right)=\delta^{i j}\left\langle S e_{i}^{M}, e_{j}^{M}\right\rangle \hat{\mathbf{n}} .
$$


Using that $S$ is a scalar multiplication by $-1 / r$ and the identity $\delta^{i j}\left\langle e_{i}^{M}, e_{j}^{M}\right\rangle=2 r^{2}$ gives

$$
\delta^{i j} B\left(e_{i}^{M}, e_{j}^{M}\right)=-2 r .
$$

Using (4.37), (4.41), and (4.43) to write (4.8) gives

$$
\tau_{F} \frac{\sqrt{h}}{\sqrt{g}}-k \delta^{i j} B\left(e_{i}^{M}, e_{j}^{M}\right)=-\left(\nu^{3}-\frac{r}{4} \pi-2 r k\right) \hat{\mathbf{n}},
$$

which vanishes since $r=\nu^{3} /(1 / 4 \pi+2 k)$. This shows that $S_{r}^{2}$ is a critical sphere.

Proof of Theorem 4.2. This is a direct calculation. Set $E_{d}(t)=E_{d}[\Phi(\cdot, t)]$ and $E_{h}(t)=$ $E_{h}[\Phi(\cdot, t)]$. By the definition of harmonic energy, we have

$$
\begin{aligned}
E_{h}^{\prime}(t) & =\frac{d}{d t} \frac{1}{2} \int_{M} g^{i j}\left\langle\frac{\partial \Phi}{\partial x^{i}}(x, t), \frac{\partial \Phi}{\partial x^{j}}(x, t)\right\rangle d v o l_{g} \\
& =\int_{M} g^{i j}\left\langle\frac{\partial \Phi}{\partial x^{i}}(x, t), \frac{\partial^{2} \Phi}{\partial t \partial x^{j}}(x, t)\right\rangle d v o l_{g} .
\end{aligned}
$$

Evaluating at $t=0$ yields

$$
E_{h}^{\prime}(0)=\int_{M} g^{i j}\left\langle\frac{\partial F}{\partial x^{i}}, \frac{\partial V}{\partial x^{j}}\right\rangle d v o l_{g}=\int_{M}\left\langle\Delta_{g} F, V\right\rangle d v o l_{g},
$$

where the last equality follows by integrating by parts. Adding this to the first variation of $E_{d}$ computed in Theorem 3.3 gives formula (4.7).

We now prove the second statement of the theorem, that is, that $F$ is a critical embedding if and only if $F: M \rightarrow F(M)$ is a harmonic map and satisfies (4.8). For $F$ to be a critical embedding, the derivative $E^{\prime}(0)$ must vanish for all $V$. This is equivalent to the condition

$$
\tau_{F} \frac{\sqrt{h}}{\sqrt{g}}+k \Delta_{g} F=0
$$

where the difference in the volume element between the two integrals in (4.7) is expressed in terms of the quotient $\sqrt{h} / \sqrt{g}$. Since $\tau_{F}$ is normal to $F(M)$, the tangential component of $\Delta_{g} F$ is zero,

$$
\left(\Delta_{g} F\right)_{\|}=0
$$

Therefore, a critical embedding $F$ is harmonic, considered as a map from $M$ onto its image, that is, $F: M \rightarrow F(M)$ [21, page 138].

We now compute the normal component of $\Delta_{g} F$. Let $V \in N_{F}$. We use (4.6) to compute $\left\langle\Delta_{g} F, V\right\rangle$ taking into account that $\left\langle\partial F / \partial x^{i}, V\right\rangle=0$. We have

$$
\left\langle\Delta_{g} F, V\right\rangle=-g^{i j}\left\langle\frac{\partial^{2} F}{\partial x^{i} \partial x^{j}}, V\right\rangle=-g^{i j}\left\langle B\left(\frac{\partial F}{\partial x^{i}}, \frac{\partial F}{\partial x^{j}}\right), V\right\rangle
$$


by the definition of second fundamental form. Since this equality holds for every $V \in N_{F}$, we get

$$
\left(\Delta_{g} F\right)_{\perp}=-g^{i j} B\left(\frac{\partial F}{\partial x^{i}}, \frac{\partial F}{\partial x^{j}}\right)=-\delta^{i j} B\left(e_{i}^{M}, e_{j}^{M}\right)
$$

Substituting into (4.47) establishes (4.8).

We close this section with a technical lemma that will be needed.

Lemma 4.7. Let $F_{0} \in C^{\infty}\left(S^{1}, \mathbb{R}^{n}\right)$. Then there exists $\epsilon>0$ such that for all $F \in C^{\infty}\left(S^{1}, \mathbb{R}^{n}\right)$ with $\left\|F-F_{0}\right\|_{C^{1}}<\epsilon$ there exist $V \in N_{F_{0}}$ and a diffeomorphism $\phi: S^{1} \rightarrow S^{1}$ such that

$$
F=\left(F_{0}+V\right) \circ \phi
$$

Moreover, $F$ has constant speed; that is, $\left|F^{\prime}\right|^{\prime}=0$ if and only if $\phi$, when viewed as a realvalued function, has its inverse given by the formula

$$
\phi^{-1}(u)=\frac{1}{\left|F^{\prime}\right|} \int_{0}^{u}\left|F_{0}^{\prime}(v)+V^{\prime}(v)\right| d v .
$$

Proof. Let $V=F-F_{0} \circ \lambda_{F_{0}} \circ F$. Since $\lambda_{F_{0}}$ assigns to $F(u) \in \Omega$ a point $\lambda_{F_{0}}(F(u)) \in S^{1}$ such that $F_{0}\left(\lambda_{F_{0}}(F(u))\right)$ realizes the distance between $F(u)$ and $F_{0}\left(S^{1}\right)$, we have that $V(u)$ is normal to $F_{0}\left(S^{1}\right)$ at $F_{0}\left(\lambda_{F_{0}} \circ F(u)\right)$, that is, $V(u) \in N_{F_{0}\left(\lambda_{F_{0}} \circ F(u)\right)}$. We also have

$$
F=F_{0} \circ \lambda_{F_{0}} \circ F+V .
$$

To prove the lemma, it suffices to show that the map $\phi: S^{1} \rightarrow S^{1}$ defined by $\phi=\lambda_{F_{0}} \circ F$ is a diffeomorphism. For this, we apply the following sublemma.

Sublemma 4.8. Let $F_{0} \in C^{\infty}\left(S^{1}, \mathbb{R}^{n}\right)$. Then there exists $\epsilon>0$ such that for all $u_{0} \in S^{1}$ and all $F \in C^{\infty}\left(S^{1}, \mathbb{R}^{n}\right)$ with $\left\|F-F_{0}\right\|_{C^{1}}<\epsilon$, the angle between $\mathbf{T}_{0}\left(u_{0}\right)$ and $\mathbf{T}(u)$ is less than $\pi / 2$, for all $u \in \phi^{-1}\left\{u_{0}\right\}$.

Proof of Sublemma 4.8. For $F$ in a $C^{1}$ neighborhood of $F_{0}$ and $u \in \phi^{-1}\left\{u_{0}\right\}$, we show that

$$
\left|\mathbf{T}_{0}\left(u_{0}\right)-\mathbf{T}(u)\right|<\sqrt{2} .
$$

Let $\epsilon_{1}>0$ such that if $\left\|F-F_{0}\right\|_{C^{1}}<\epsilon_{1}$, then $\left\|\mathbf{T}-\mathbf{T}_{0}\right\|_{C^{0}}<1 / \sqrt{2}$. Since $\mathbf{T}_{0} \circ \lambda_{F_{0}}: \Omega \rightarrow \mathbb{R}^{n}$ is a uniformly continuous function on the compact $\Omega$, there exists $\epsilon_{2}>0$ such that for all $\mathbf{x}_{1}, \mathbf{x}_{2} \in \Omega$ with $\left|\mathbf{x}_{1}-\mathbf{x}_{2}\right|<\epsilon_{2}$, we have $\left|\mathbf{T}_{0} \circ \lambda_{F_{0}}\left(\mathbf{x}_{1}\right)-\mathbf{T}_{0} \circ \lambda_{F_{0}}\left(\mathbf{x}_{2}\right)\right|<1 / \sqrt{2}$. Setting $\epsilon=\min \left\{\epsilon_{1}, \epsilon_{2}\right\}$, we have

$$
\begin{aligned}
\left|\mathbf{T}_{0}\left(u_{0}\right)-\mathbf{T}(u)\right| & =\left|\mathbf{T}_{0}(\phi(u))-\mathbf{T}(u)\right| \\
& \leq\left|\mathbf{T}_{0}(\phi(u))-\mathbf{T}_{0}\left(\lambda_{F_{0}} \circ F_{0}(u)\right)\right|+\left|\mathbf{T}_{0}\left(\lambda_{F_{0}} \circ F_{0}(u)\right)-\mathbf{T}(u)\right| .
\end{aligned}
$$

Writing $\phi=\lambda_{F_{0}} \circ F$, and since $\left|F(u)-F_{0}(u)\right|<\epsilon \leq \epsilon_{2}$, we get

$$
\left|\mathbf{T}_{0}\left(u_{0}\right)-\mathbf{T}(u)\right|<\frac{1}{\sqrt{2}}+\left|\mathbf{T}_{0}\left(\lambda_{F_{0}} \circ F_{0}(u)\right)-\mathbf{T}(u)\right| .
$$


Since $\lambda_{F_{0}} \circ F_{0}: S^{1} \rightarrow S^{1}$ is the identity map,

$$
\left|\mathbf{T}_{0}\left(u_{0}\right)-\mathbf{T}(u)\right|<\frac{1}{\sqrt{2}}+\left|\mathbf{T}_{0}(u)-\mathbf{T}(u)\right|
$$

Using $\left|\mathbf{T}_{0}(u)-\mathbf{T}(u)\right|<1 / \sqrt{2}$ gives (4.54).

We now continue with the proof of Lemma 4.7. We use Sublemma 4.8 to prove that $\phi$ is a diffeomorphism.

We start by showing that $\phi$ is injective. Let $u_{0} \in S^{1}$ and consider the subset $\left\{u_{i}\right\}_{i \in I} \subset S^{1}$ such that $\phi\left(u_{i}\right)=u_{0}$. Then $u_{i} \in \phi^{-1}\left\{u_{0}\right\}$ and $F\left(u_{i}\right) \in \lambda_{F_{0}}^{-1}\left\{u_{0}\right\}$ for all $i \in I$. Consider now the plane $N_{F_{0}\left(u_{0}\right)}$ orthogonal to $F_{0}\left(S^{1}\right)$ at $F_{0}\left(u_{0}\right)$. Then $\left\{F\left(u_{i}\right)\right\} \subset N_{F_{0}\left(u_{0}\right)}$ is the set of points

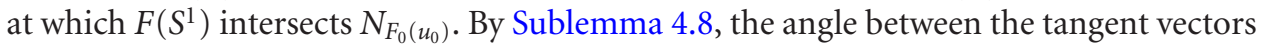
$\mathbf{T}_{0}\left(u_{0}\right)$ and $\mathbf{T}\left(u_{i}\right)$ is less than $\pi / 2$, for all $i \in I$. This can only happen if $\left\{F\left(u_{i}\right)\right\}$ consists of one element only. This shows that $\phi$ is injective.

Next we show that $\phi$ is surjective. Since $\phi=\lambda_{F_{0}} \circ F$ is continuous and since $S^{1}$ is Hausdorff and compact, by the closed map lemma, $\phi\left(S^{1}\right)$ is closed. In particular, we have that $\phi\left(S^{1}\right)$ contains its boundary $\partial \phi\left(S^{1}\right) \subset S^{1}$. Assume that $\partial \phi\left(S^{1}\right) \neq \varnothing$ and let $u_{1} \in S^{1}$ with $\phi\left(u_{1}\right) \in \partial \phi\left(S^{1}\right)$. Set $u_{0}=\phi\left(u_{1}\right)$. Then $u_{1} \in \phi^{-1}\left\{u_{0}\right\}$, and by Sublemma 4.8, the angle between $\mathbf{T}_{0}\left(u_{0}\right)$ and $\mathbf{T}\left(u_{1}\right)$ is less than $\pi / 2$. In particular, there is an open segment near $F\left(u_{1}\right)$ in $F\left(S^{1}\right)$ that projects onto an open segment of $F_{0}\left(u_{0}\right)$ in $F_{0}\left(S^{1}\right)$. This contradicts that $\phi\left(u_{1}\right) \in \partial \phi\left(S^{1}\right)$, and then $\partial \phi\left(S^{1}\right)=\varnothing$. This shows that $\phi$ is surjective.

From the homeomorphism theorem, it follows that the continuous bijection $\phi: S^{1} \rightarrow$ $S^{1}$ from the compact and Hausdorff space $S^{1}$ onto itself is a homeomorphism. A sufficient condition for a smooth homeomorphism to be a diffeomorphism is that its differential be nonsingular at every point of $S^{1}$. Since $F$ is an embedding, to show that $\phi_{*}=\left(\lambda_{F_{0}} \circ F\right)_{*}$ is injective, it suffices to show that $\lambda_{F_{0} *} \mathbf{T}(u) \neq 0$, for all $u \in S^{1}$. If $\lambda_{F_{0} *} \mathbf{T}\left(u_{1}\right)=0$ for some $u_{1} \in S^{1}$, the angle between $\mathbf{T}\left(u_{1}\right)$ and $\mathbf{T}_{0}\left(\lambda_{F_{0}} \circ F\left(u_{1}\right)\right)$ would be $\pi / 2$. This contradicts Sublemma 4.8, and therefore $\lambda_{F_{0} *} \mathbf{T}(u) \neq 0$ for all $u \in S^{1}$. This shows that $\phi$ is a diffeomorphism.

We now prove the second statement of the lemma, that is, that $F$ has constant speed if and only if $\phi^{-1}$ is given by (4.52).

View $\phi$ as a real-valued function defined on the interval $[0,2 \pi]$. Without loss of generality, let $\phi(0)=0$. We compute $\left|F^{\prime}\right|$ by applying the chain rule in (4.51). This gives $\left|F^{\prime}\right|=\left|\left(F_{0}^{\prime}+V^{\prime}\right) \circ \phi\right|\left|\phi^{\prime}\right|$. Since $\phi$ is a diffeomorphism and $\phi(0)=0, \phi^{\prime}>0$. Hence

$$
\left|F^{\prime}\right|=\left|\left(F_{0}^{\prime}+V^{\prime}\right) \circ \phi\right| \phi^{\prime}
$$

We use the formula for the derivative of the inverse function $\left(\phi^{-1}\right)^{\prime} \circ \phi=1 / \phi^{\prime}$ and (4.58) to obtain $\left(\phi^{-1}\right)^{\prime} \circ \phi=\left(1 /\left|F^{\prime}\right|\right)\left|F_{0}^{\prime}+V^{\prime}\right| \circ \phi$. Composing with $\phi$ on the right and integrating from 0 to $u \in[0,2 \pi]$ gives

$$
\phi^{-1}(u)-\phi^{-1}(0)=\int_{0}^{u} \frac{\left|F_{0}^{\prime}(v)+V^{\prime}(v)\right|}{\left|F^{\prime}(v)\right|} d v,
$$


since $\phi(0)=0$. If $\left|F^{\prime}\right|$ is constant, then this formula implies (4.52). Conversely, assume that (4.52) holds. Then we have

$$
\int_{0}^{u} \frac{\left|F_{0}^{\prime}(v)+V^{\prime}(v)\right|}{\left|F^{\prime}(v)\right|} d v=\frac{1}{\left|F^{\prime}(u)\right|} \int_{0}^{u}\left|F_{0}^{\prime}(v)+V^{\prime}(v)\right| d v .
$$

By taking the derivative with respect to $u$ and simplifying, we get $\left|F^{\prime}\right|^{\prime}=0$. This proves the lemma.

4.3. Second variation of $\mathbf{E}$. We now investigate conditions under which a critical embedding yields a local minimum of $E$ by computing its second variation.

Denote by $g^{*}$ the induced metric in the cotangent bundle $T_{x}^{*} M$. Our formulation of the second variation of $E$ involves two additional quantities:

(i) the symmetric functional $Q_{F}: C^{\infty}\left(M, \mathbb{R}^{n}\right) \times C^{\infty}\left(M, \mathbb{R}^{n}\right) \rightarrow C^{\infty}(M)$ given by

$$
Q_{F}(X, Y)=k \delta^{i j}\left\langle d X\left(e_{i}^{M}\right), d Y\left(e_{j}^{M}\right)\right\rangle-H_{F}(X, Y) \frac{\sqrt{h}}{\sqrt{g}}
$$

(ii) the bilinear map $q_{F}:\left(\mathbb{R}^{n} \otimes T^{*} M\right) \times\left(\mathbb{R}^{n} \otimes T^{*} M\right) \rightarrow C^{\infty}(M)$ given by

$$
\begin{aligned}
q_{F}(X \otimes \xi, Y \otimes \eta)= & k\langle X, Y\rangle g^{*}(\xi, \eta) \\
& -\int_{\Omega_{F}}\langle X, v\rangle\langle Y, v\rangle\left\langle\xi^{\#},\left(I-S_{v}\right)^{-1} \eta^{\#}\right\rangle p(F+v) \operatorname{det}\left(I-S_{v}\right) d v \frac{\sqrt{h}}{\sqrt{g}} .
\end{aligned}
$$

Our main goal in this section is to prove the following.

TheOREM 4.9. Let $F$ be a critical embedding of $E$ and let $\Phi$ be a smooth variation of $F$. Then the second variation of $E$ is given by the formula

$$
\left.\frac{\partial^{2} E[\Phi(\cdot, s, t)]}{\partial s \partial t}\right|_{(s, t)=0}=\int_{M} P_{F}\langle V, W\rangle d v o l_{h}+\int_{M} Q_{F}(V, W) d v o l_{g},
$$

where $V$ and $W$ are the variational vector fields generated by $\Phi$. Moreover, if $P_{F}>0$, the bilinear map $q_{F}$ is positive definite, and $M=S^{1}$, then there is an $\epsilon>0$ such that all $\tilde{F} \in \mathscr{E}_{\Omega}$ with $\|F-\tilde{F}\|_{C^{2}}<\epsilon$ satisfy $E[F] \leq E[\tilde{F}]$.

Theorem 4.10. Consider the standard sphere $S^{2} \subset \mathbb{R}^{3}$ with the induced Euclidean metric. Let $F: S^{2} \rightarrow S^{2} \subset \mathbb{R}^{3}$ be a critical embedding of $E$. If $q_{F}$ is positive semidefinite, then the second variation of $E$ is positive semidefinite.

Before proving Theorems 4.9 and 4.10, we discuss some of their consequences.

Example 4.11 (curves in $\mathbb{R}^{n}$ ). In this case $q_{F}$ is positive definite if and only if

$$
k|X|^{2}-\frac{\Lambda_{2}(X, X)}{\left|F^{\prime}\right|}>0, \quad X \in \mathbb{R}^{n} .
$$


To verify this we compute formula (4.62) using the identities

$$
\left(I-S_{v}\right)^{-1} \operatorname{det}\left(I-S_{v}\right)=1, \quad\left\langle\xi^{\#}, \eta^{\#}\right\rangle=\frac{g^{*}(\xi, \eta)}{\left|F^{\prime}\right|^{2}}, \quad \frac{\sqrt{h}}{\sqrt{g}}=\left|F^{\prime}\right| .
$$

We get

$$
\begin{aligned}
q_{F}(X \otimes \xi, Y \otimes \eta)= & k\langle X, Y\rangle g^{*}(\xi, \eta) \\
& -\int_{\Omega_{F}}\langle X, v\rangle\langle Y, v\rangle g^{*}(\xi, \eta)\left|F^{\prime}\right|^{-2} p(F+v) d v\left|F^{\prime}\right| .
\end{aligned}
$$

Simplifying and writing the result in terms of $\Lambda_{2}$ gives

$$
q_{F}(X \otimes \xi, Y \otimes \eta)=g^{*}(\xi, \eta)\left(k\langle X, Y\rangle-\frac{\Lambda_{2}(X, Y)}{\left|F^{\prime}\right|}\right),
$$

from which the statement follows.

Example 4.12. Let $p$ be a radial density relative to a curve $\Gamma$. From Example 2.8, it follows that the covariance tensor $\sigma_{\perp}^{2}$ is a constant multiple $\left|\sigma_{\perp}^{2}\right|$ of the Euclidean scalar product restricted to $\Omega_{F(u)}$. If $k=2 \pi\left|\sigma_{\perp}^{2}\right| / L_{F}^{2}$, then $\Gamma$ is a critical curve by Example 4.4. In this case, we have

$$
q_{F}(X \otimes \xi, Y \otimes \eta)=k\langle\mathbf{T}, X\rangle\langle\mathbf{T}, Y\rangle g^{*}(\xi, \eta)
$$

To verify this, use the identities $\Lambda_{0}=1 / L_{F}, \mu_{\perp}=0,\left|F^{\prime}\right|=L_{F} / 2 \pi$, and $k=2 \pi\left|\sigma_{\perp}^{2}\right| / L_{F}^{2}$, and write $\Lambda_{2}$ in terms of $\sigma_{\perp}^{2}$ in (4.67). We have

$$
\begin{aligned}
q_{F}(X \otimes \xi, Y \otimes \eta) & =g^{*}(\xi, \eta)\left(k\langle X, Y\rangle-\frac{\Lambda_{0}\left(\sigma_{\perp}^{2}+\mu_{\perp} \otimes \mu_{\perp}\right)(X, Y)}{\left|F^{\prime}\right|}\right) \\
& =g^{*}(\xi, \eta)\left(\frac{2 \pi\left|\sigma_{\perp}^{2}\right|\langle X, Y\rangle}{L_{F}^{2}}-\left(\frac{1}{L_{F}}\right) \frac{\sigma_{\perp}^{2}(X, Y)}{L_{F} / 2 \pi}\right) .
\end{aligned}
$$

Since the covariance tensor is a constant multiple $\left|\sigma_{\perp}^{2}\right|$ of the Euclidean scalar product restricted to $\Omega_{F(u)}$, we get that the scalar product of the normal components $\left\langle X_{\perp}, Y_{\perp}\right\rangle$ cancels (here $X_{\perp}=X-\langle\mathbf{T}, X\rangle \mathbf{T}$ ). This implies the assertion.

Example 4.13. For critical curves in the plane, we write

$$
\mu_{\perp}(\mathbf{N}(u))=\mu_{\perp}(u), \quad \sigma_{\perp}^{2}(\mathbf{N}(u), \mathbf{N}(u))=\sigma_{\perp}^{2}(u) .
$$

Then $q_{F}$ is positive definite if and only if

$$
k>\frac{2 \pi \Lambda_{0}\left(\mu_{\perp}^{2}+\sigma_{\perp}^{2}\right)}{L_{F}} .
$$

In particular, for an annular distribution, this condition is satisfied if and only if the radius of a critical circle is less than $1 /\left(2 \pi \nu^{0}\right)$, where $\nu^{0}$ is defined by $(4.24)$. 
To verify this, we use the identity $\left|F^{\prime}\right|=L_{F} / 2 \pi$, write $X=\langle\mathbf{T}, X\rangle \mathbf{T}+\langle\mathbf{N}, X\rangle \mathbf{N}$, and write $\Lambda_{2}$ in terms of $\mu_{\perp}$ and $\sigma_{\perp}^{2}$ in (4.67). We have

$$
\begin{aligned}
q_{F}(X \otimes \xi, X \otimes \xi) & =g^{*}(\xi, \xi)\left(k|X|^{2}-\frac{\Lambda_{0}\left(\sigma_{\perp}^{2}+\mu_{\perp} \otimes \mu_{\perp}\right)(X, X)}{\left|F^{\prime}\right|}\right) \\
& =g^{*}(\xi, \xi)\left(k\left(\langle\mathbf{T}, X\rangle^{2}+\langle\mathbf{N}, X\rangle^{2}\right)-\frac{\Lambda_{0}\left(\sigma_{\perp}^{2}+\mu_{\perp}^{2}\right)\langle\mathbf{N}, X\rangle^{2}}{L_{F} / 2 \pi}\right) .
\end{aligned}
$$

This is positive for all $X \in T_{u} S^{1}, X \neq 0$, if and only if $k>2 \pi \Lambda_{0}\left(\mu_{\perp}^{2}+\sigma_{\perp}^{2}\right) / L_{F}$. Now set $X=d \mathbf{T} / d s$ in (4.9). We get

$$
-\Lambda_{1}\left(\frac{d \mathbf{T}}{d s}\right)+\Lambda_{2}\left(\frac{d \mathbf{T}}{d s}, \frac{d \mathbf{T}}{d s}\right)-\left(\frac{k L_{F}}{2 \pi}\right)\left\langle\frac{d \mathbf{T}}{d s}, \frac{d \mathbf{T}}{d s}\right\rangle=0 .
$$

By (4.67), we have that $q_{F}$ is positive definite if and only if $-\Lambda_{1}(d \mathbf{T} / d s)>0$. For a critical circle with radius $r$ in an annular distribution, we have, by the definition of $\Lambda_{1}$,

$$
-\Lambda_{1}\left(\frac{d \mathbf{T}}{d s}\right)=-\int_{r_{1}-r}^{r_{2}-r}\left\langle\frac{d \mathbf{T}}{d s}, v \mathbf{N}\right\rangle p(r+v) d v
$$

Using $\langle d \mathbf{T} / d s, \mathbf{N}\rangle=-1 / r$ and making the change of variables $\rho=r+v$ gives

$$
-\Lambda_{1}\left(\frac{d \mathbf{T}}{d s}\right)=-\int_{r_{1}}^{r_{2}}\left(-\frac{1}{r}\right)(\rho-r) p(\rho) d \rho .
$$

Simplifying and writing the result in terms of $\nu^{k}$ gives

$$
-\Lambda_{1}\left(\frac{d \mathbf{T}}{d s}\right)=\frac{\nu^{1}-r \nu^{0}}{r}
$$

Since $v^{1}=1 / 2 \pi$ (Example 4.5), we get that $q_{F}$ is positive definite if and only if $r<$ $1 /\left(2 \pi v^{0}\right)$.

Example 4.14 the spherical shell. In Example 4.6 we showed that the sphere $S_{r}^{2}$ of radius $r=\nu^{3} /(2 k+1 / 4 \pi)$ is critically embedded in $\Omega$. For this, the second variation of $E$ is positive semidefinite if the quadratic form

$$
k\langle X, Y\rangle-\left(\Lambda_{0}\left(\mu_{\perp} \otimes \mu_{\perp}+\sigma_{\perp}^{2}\right)+\frac{\Lambda_{3}(\hat{\mathbf{n}})}{r}\right)(X, Y), \quad X, Y \in \mathbb{R}^{n}
$$

is positive semidefinite. To verify this claim, we compute $q_{F}$ and apply Theorem 4.10.

We substitute the identities

$$
\left\langle\xi^{\#}, \eta^{\#}\right\rangle=\frac{g^{*}(\xi, \eta)}{r^{2}}, \quad\left(I-S_{v}\right)^{-1} \operatorname{det}\left(I-S_{v}\right)=1+\frac{v}{r}, \quad \frac{\sqrt{h}}{\sqrt{g}}=r^{2}
$$

in definition (4.62) of $q_{F}$ to obtain

$$
q_{F}(X \otimes \xi, Y \otimes \eta)=k\langle X, Y\rangle g^{*}(\xi, \eta)-\int_{\Omega_{F}} g^{*}(\xi, \eta) r^{-2}\left(1+\frac{v}{r}\right) p(F+v) d v r^{2} .
$$


We write $v=\langle v \hat{\mathbf{n}}, \hat{\mathbf{n}}\rangle$ and use the definition of transverse moments $\Lambda_{k}$ to get

$$
q_{F}(X \otimes \xi, Y \otimes \eta)=k\langle X, Y\rangle g^{*}(\xi, \eta)-g^{*}(\xi, \eta)\left(\Lambda_{2}(X, Y)+\Lambda_{3}\left(X, Y, \frac{\hat{\mathbf{n}}}{r}\right)\right)
$$

Writing $\Lambda_{2}$ in terms of the transverse mean $\mu_{\perp}$ and the covariance tensor $\sigma_{\perp}^{2}$ gives (4.77) multiplied by the factor $g^{*}(\xi, \eta)$. Therefore $q_{F}$ is positive semidefinite if and only if $(4.77)$ defines a positive semidefinite quadratic form.

Example 4.15 (embedded surfaces in $\mathbb{R}^{3}$ ). We write the second variation of the functional $E$ for the case when the manifold is an embedded surface in $\mathbb{R}^{3}$. The second variation of $E$ is expressed in terms of the function $P_{F}$ and of $Q_{F}$. The following corollary to Theorem 4.9 states that if the variational vector fields are normal to the embedded surface and if a certain quadratic form that depends on the spring constant, the metric of the surface, the Euclidean metric induced in the surface, the second and third moments, and the shape operator is positive definite, then the second variation is positive definite. The meaning of this corollary is that a necessary condition for an embedding to be a minimum of the functional $E$ is that the spring constant is sufficiently large so that this quadratic form is positive definite.

Let $\hat{\mathbf{n}}$ denote the outer unit vector to $M$ and let the second variation of the functional $E$ involve the shape operator. The following is the definition of an operation of $2 \times 2$ matrices. This operation is applied to the shape operator in the calculation of the second variation.

Corollary 4.16. Suppose that

(1) $X$ and $Y$ are normal to $F(M)$,

(2) for almost all $x \in M, k g^{i j}-(\sqrt{h} / \sqrt{g}) h^{i k}\left(\mu^{2} \delta_{k}^{j}-\mu^{3}\left(S^{\dagger}\right)_{k}^{j}\right)$ is positive definite.

Then $\partial^{2} E[\Phi(\cdot, s, t)] /\left.\partial s \partial t\right|_{(s, t)=0}$ is positive definite.

In the proof of Corollary 4.16 one computes $Q_{F}$ in terms of the quadratic form in the statement of the corollary evaluated at the gradients of the magnitudes of the variational vector fields. For it, the partial derivatives of the variational vector fields are computed in local coordinates. On the other hand, the function $P_{F}$ is positive almost everywhere since the determinant $\operatorname{det}\left(I-S_{v}\right)$ is nonvanishing in $\Omega$ and since, by hypothesis, the quadratic form is positive definite; Theorem 4.9 implies that the second variation of $E$ is positive definite.

Proof of Corollary 4.16. By Hypothesis (1),

$$
\frac{\partial X}{\partial x^{i}}=\frac{\partial\langle\hat{\mathbf{n}}, X\rangle}{\partial x^{i}} \hat{\mathbf{n}}+\langle\hat{\mathbf{n}}, X\rangle \frac{\partial \hat{\mathbf{n}}}{\partial x^{i}} .
$$

Therefore,

$$
\left\langle\frac{\partial X}{\partial x^{i}}, \frac{\partial Y}{\partial x^{j}}\right\rangle=\frac{\partial\langle\hat{\mathbf{n}}, X\rangle}{\partial x^{i}} \frac{\partial\langle\hat{\mathbf{n}}, Y\rangle}{\partial x^{j}}+\left\langle\langle\hat{\mathbf{n}}, X\rangle \frac{\partial \hat{\mathbf{n}}}{\partial x^{i}},\langle\hat{\mathbf{n}}, Y\rangle \frac{\partial \hat{\mathbf{n}}}{\partial x^{j}}\right\rangle
$$


since $\left\langle\hat{\mathbf{n}}, \partial \hat{\mathbf{n}} / \partial x^{i}\right\rangle=0$. By (4.82), (4.61), and Hypothesis (1),

$$
\begin{aligned}
Q_{F}(X, Y)= & k g^{i j}\left\langle\frac{\partial X}{\partial x^{i}}, \frac{\partial Y}{\partial x^{j}}\right\rangle-\left\langle\operatorname{grad}\langle X, \hat{\mathbf{n}}\rangle,\left(\mu^{2} I-\mu^{3} S^{\dagger}\right) \operatorname{grad}\langle Y, \hat{\mathbf{n}}\rangle\right\rangle \frac{\sqrt{h}}{\sqrt{g}} \\
= & k g^{i j}\left(\frac{\partial\langle\hat{\mathbf{n}}, X\rangle}{\partial x^{i}} \frac{\partial\langle\hat{\mathbf{n}}, Y\rangle}{\partial x^{j}}+\left\langle\langle\hat{\mathbf{n}}, X\rangle \frac{\partial \hat{\mathbf{n}}}{\partial x^{i}},\langle\hat{\mathbf{n}}, Y\rangle \frac{\partial \hat{\mathbf{n}}}{\partial x^{j}}\right\rangle\right) \\
& -\frac{\partial\langle X, \hat{\mathbf{n}}\rangle}{\partial x^{i}} h^{i j}\left(\mu^{2} I-\mu^{3} S^{\dagger}\right)_{j}^{k} \frac{\partial\langle Y, \hat{\mathbf{n}}\rangle}{\partial x^{k}} \frac{\sqrt{h}}{\sqrt{g}} .
\end{aligned}
$$

The function $P_{F}$ is positive almost everywhere since the determinant $\operatorname{det}\left(I-S_{v}\right)$ is nonvanishing in $\Omega$. Then, by (4.83), Hypothesis (2), and Theorem 4.9, $\partial^{2} E[\Phi(\cdot, s, t)] /\left.\partial s \partial t\right|_{(s, t)=0}$ is positive definite.

Proof of Theorem 4.9. By (4.45),

$$
\begin{aligned}
\frac{\partial^{2} E_{h}(s, t)}{\partial s \partial t} & =\frac{\partial}{\partial s} \int_{M} g^{i j}\left\langle\frac{\partial \Phi}{\partial x^{i}}(x, s, t), \frac{\partial^{2} \Phi}{\partial t \partial x^{j}}(x, s, t)\right\rangle d v o l_{g} \\
& =\int_{M} g^{i j}\left(\left\langle\frac{\partial^{2} \Phi}{\partial s \partial x^{i}}(x, s, t), \frac{\partial^{2} \Phi}{\partial t \partial x^{j}}(x, s, t)\right\rangle+\left\langle\frac{\partial \Phi}{\partial x^{i}}(x, s, t), \frac{\partial^{3} \Phi}{\partial s \partial t \partial x^{j}}(x, s, t)\right\rangle\right) d v o l_{g} .
\end{aligned}
$$

Evaluation at $s, t=0$ gives

$$
\begin{aligned}
\left.\frac{\partial^{2} E_{h}}{\partial s \partial t}\right|_{(s, t)=0} & =\int_{M} g^{i j}\left(\left\langle\frac{\partial V}{\partial x^{i}}, \frac{\partial W}{\partial x^{j}}\right\rangle+\left\langle\frac{\partial F}{\partial x^{i}}, \frac{\partial Z}{\partial x^{j}}\right\rangle\right) d v o l_{g} \\
& =\int_{M}\left(\delta^{i j}\left\langle d V\left(e_{i}^{M}\right), d W\left(e_{j}^{M}\right)\right\rangle+\left\langle\Delta_{g} F, Z\right\rangle\right) d v o l_{g}
\end{aligned}
$$

where $Z=\left.\left(\partial^{2} \Phi / \partial s \partial t\right)\right|_{(s, t)=0}$, and we integrated by parts in the last step. Adding this to the second derivative of $E_{d}$ (formula (3.78)) gives

$$
\begin{aligned}
\left.\frac{\partial^{2} E[\Phi(\cdot, s, t)]}{\partial s \partial t}\right|_{(s, t)=0}= & \left.\frac{\partial^{2} E_{d}[\Phi(\cdot, s, t)]}{\partial s \partial t}\right|_{(s, t)=0}+\left.k \frac{\partial^{2} E_{h}[\Phi(\cdot, s, t)]}{\partial s \partial t}\right|_{(s, t)=0} \\
= & \int_{M}\left(P_{F}\langle V, W\rangle-H_{F}(V, W)+\left\langle\tau_{F}, Z\right\rangle\right) d v o l_{h} \\
& +k \int_{M}\left(\delta^{i j}\left\langle d V\left(e_{i}^{M}\right), d W\left(e_{j}^{M}\right)\right\rangle+\left\langle\Delta_{g} F, Z\right\rangle\right) d v o l_{g}
\end{aligned}
$$

Since $F$ is a critical embedding, Theorem 4.2 implies that the terms involving $Z$ cancel. Writing (4.86) in terms of $Q_{F}$ establishes (4.63).

Next we prove the second statement of the theorem, that is, the sufficient conditions for a local minimum of $E$. We have the following proposition. 
Proposition 4.17. There is a neighborhood $U_{0}$ of $F_{0}$ in $C^{2}\left(S^{1}, \mathbb{R}^{n}\right)$ such that for any embedding $F$ in this neighborhood satisfying $\left|F^{\prime}\right|^{\prime}=0$, there exists $V \in N_{F_{0}}$ such that $F=$ $\left(F_{0}+V\right) \circ \phi$, where $\phi: S^{1} \rightarrow S^{1}$ is a diffeomorphism that, viewed as a real-valued function, has its inverse defined by (4.52). Moreover, for all such embeddings $F \in U_{0}$, there exist $C, \epsilon>0$ such that if $\left\|F-F_{0}\right\|_{C^{2}}<\epsilon$, then the second variation of $E$ at $F$ satisfies $\delta^{2} E[F] \geq C\|V\|_{1}^{2}$, where $\|V\|_{1}^{2}$ is the square of the Sobolev norm of $V$.

Proof of Proposition 4.17. This is a consequence of Lemma 4.7. To prove the "moreover" part we let $W \in C^{\infty}\left(S^{1}, \mathbb{R}^{n}\right)$ be a variational vector field. We compute $\delta^{2} E(W, W)$ as

$$
\begin{aligned}
\delta^{2} E(W, W)=\int_{S^{1}}\{ & P_{F}\left\langle W_{\perp}, W_{\perp}\right\rangle\left|F^{\prime}\right|+k\left(\left\langle\mathbf{T}, \frac{W_{\|}^{\prime}}{\left|F^{\prime}\right|}\right\rangle-\left\langle\frac{\mathbf{T}^{\prime}}{\left|F^{\prime}\right|}, W_{\perp}\right\rangle\right)^{2} \\
& \left.+q_{F}\left(\nabla^{\perp} W_{\perp}, \nabla^{\perp} W_{\perp}\right)\right\} d u .
\end{aligned}
$$

We will prove this formula later; we use it now to find a lower bound of $\delta^{2} E(W, W)$. Since $q_{F_{0}}$ is positive definite, by Lemma 4.20 , there are $C_{1}, \epsilon_{1}>0$ such that for all $F \in \mathscr{E}_{\Omega}$ with $\left\|F-F_{0}\right\|_{C^{2}}<\epsilon_{1}$, we have

$$
q_{F}\left(d W_{\perp}, d W_{\perp}\right) \geq C_{1}\left|\frac{W_{\perp}^{\prime}}{\left|F^{\prime}\right|}\right|^{2}
$$

If $P_{F_{0}}>0$, by Lemma 4.18 , there are $C_{2}, \epsilon_{2}>0$ such that if $\left\|F-F_{0}\right\|_{C^{2}}<\epsilon_{2}$, then

$$
P_{F}\left|F^{\prime}\right| \geq C_{2} \text {. }
$$

Combining inequalities (4.88) and (4.89) with estimate (4.87) gives

$$
\delta^{2} E(W, W) \geq \int_{S^{1}}\left\{C_{2}\left|W_{\perp}\right|^{2}+C_{1}\left|\frac{W_{\perp}^{\prime}}{\left|F^{\prime}\right|}\right|^{2}\right\} d u \geq \min \left\{C_{1}, C_{2}\right\}\left\|W_{\perp}\right\|_{1}^{2} .
$$

Now we consider a constant speed variation $\Phi_{t}=(F+t V) \circ \phi_{t}$ and compute $W=$ $\left.\left(d \Phi_{t} / d t\right)\right|_{t=0}$. By the chain rule,

$$
\left.\frac{d \Phi_{t}}{d t}\right|_{t=0}=\left[\left(F^{\prime}+t V^{\prime}\right) \circ \phi_{t} \cdot \phi_{t}^{\prime}\right]_{t=0}+\left.V \circ \phi_{t}\right|_{t=0}=\left.F^{\prime} \phi_{t}^{\prime}\right|_{t=0}+V .
$$

Since $F^{\prime}(u) \in T_{u} S^{1}$, we get $W_{\perp}=V$. Substituting this into (4.90), we get that if $\| F-$ $F_{0} \|_{C^{2}}<\min \left\{\epsilon_{1}, \epsilon_{2}\right\}$, then the second variation $\delta^{2} E(W, W)$ is bounded below by

$$
\delta^{2} E(W, W) \geq \min \left\{C_{1}, C_{2}\right\}\|V\|_{1}^{2} .
$$

This proves Proposition 4.17.

We now continue with the proof of Theorem 4.9. We use Proposition 4.17 to define a constant speed variation of $F_{0}$ given by $\Phi_{t}=\left(F_{0}+t V\right) \circ \phi_{t}$, with $\phi_{t}$ given by (4.52). Consider the real-valued function defined by $E(t)=E\left[\Phi_{t}\right]$. By Taylor's theorem,

$$
E[F]-E\left[F_{0}\right]=E^{\prime}(0)+\int_{0}^{1}(1-t) E^{\prime \prime}(t) d t .
$$


Since $F_{0}$ is a critical embedding, then $E^{\prime}(0)=0$. Since $E^{\prime \prime} \geq C\|t V\|_{1}^{2}=C t^{2}\|V\|_{1}^{2}$, we have

$$
E[F]-E\left[F_{0}\right] \geq \frac{C\|V\|_{1}^{2}}{12} .
$$

We use this inequality to show that $F_{0}$ is a local minimum. Any $\tilde{F} \in U_{0}$ can be written as $\tilde{F}=\left(F_{0}+V\right) \circ \tilde{\phi}$ with $V \in N_{F_{0}}$ and $\tilde{\phi}: S^{1} \rightarrow S^{1}$ a diffeomorphism. Let $F$ be the constant speed embedding defined by $F=\left(F_{0}+V\right) \circ \phi$ with $\phi$ given by (4.52). We show first that $E[F] \leq E[\tilde{F}]$, and then use $(4.94)$ to conclude that $E\left[F_{0}\right] \leq E[\tilde{F}]$ for all $\tilde{F} \in U_{0}$.

Let $\gamma=\left(F_{0}+V\right)\left(S^{1}\right)$ be the image curve of $S^{1}$ under $F_{0}+V$. Since the length of $\gamma$ does not depend on the parameterization of $S^{1}$, we have

$$
\int_{0}^{2 \pi}\left|\tilde{F}^{\prime}\right| d u=L_{\gamma}=\int_{0}^{2 \pi}\left|F^{\prime}\right| d u .
$$

Since $\left|F^{\prime}\right|$ is constant, we have $\left|F^{\prime}\right|=L_{\gamma} / 2 \pi$. Hence

$$
E_{h}[F]=\frac{1}{2} \int_{0}^{2 \pi}\left|F^{\prime}\right|^{2} d u=\frac{L_{\gamma}^{2}}{4 \pi}=\frac{1}{4 \pi}\left(\int_{0}^{2 \pi}\left|\tilde{F}^{\prime}\right| d u\right)^{2}
$$

By Schwarz's inequality,

$$
E_{h}[F] \leq \frac{1}{4 \pi} \int_{0}^{2 \pi} 1^{2} d u \int_{0}^{2 \pi}\left|\tilde{F}^{\prime}\right|^{2} d u=E_{h}[\tilde{F}]
$$

Since the expected value of the distance to $\gamma$ does not depend on the parameterization of $S^{1}$, that is, $E_{d}[F]=E_{d}[\tilde{F}]$, by (4.97), we have

$$
E_{d}[F]+k E_{h}[F]=E_{d}[\tilde{F}]+k E_{h}[F] \leq E_{d}[\tilde{F}]+k E_{h}[\tilde{F}] .
$$

By (4.94), we conclude

$$
E\left[F_{0}\right] \leq E[F] \leq E[\tilde{F}]
$$

This shows that $F_{0}$ is a local minimum.

To complete the proof of Theorem 4.9 we need to prove formula (4.87). For this, we use formulas (4.137) through (4.140). We start by computing (4.138) using the identities

$$
e_{i}^{M}=\mathbf{T}, \quad d W\left(e_{i}^{M}\right)=\frac{W^{\prime}}{\left|F^{\prime}\right|}, \quad \delta^{i j} h\left(d W_{\|}\left(e_{i}^{M}\right), d W_{\|}\left(e_{j}^{M}\right)\right)=\left\langle\mathbf{T}, \frac{W_{\|}^{\prime}}{\left|F^{\prime}\right|}\right\rangle^{2} .
$$

Since $W_{\|}=\left\langle\mathbf{T}, W_{\|}\right\rangle \mathbf{T}, W_{\|}^{\prime}=\left\langle\mathbf{T}, W_{\|}\right\rangle^{\prime} \mathbf{T}+\left\langle\mathbf{T}, W_{\|}\right\rangle \mathbf{T}^{\prime}$. Hence, the normal component of $W_{\|}^{\prime}$ is given by

$$
B\left(e_{i}^{M}, W_{\|}\right)=\left\langle\mathbf{T}, W_{\|}\right\rangle \frac{\mathbf{T}^{\prime}}{\left|F^{\prime}\right|}
$$

This implies that

$$
B\left(W_{\|}, W_{\|}\right)=\left\langle\mathbf{T}, W_{\|}\right\rangle^{2} \frac{\mathbf{T}^{\prime}}{\left|F^{\prime}\right|}, \quad B\left(e_{i}^{M}, e_{j}^{M}\right)=\frac{\mathbf{T}^{\prime}}{\left|F^{\prime}\right|} .
$$


By the Gauss equation [3, page 135],

$$
R_{F}\left(W_{\|}, e_{i}^{M}, e_{j}^{M}, W_{\|}\right)=\left\langle B\left(W_{\|}, W_{\|}\right), B\left(e_{i}^{M}, e_{j}^{M}\right)\right\rangle-\left\langle B\left(W_{\|}, e_{i}^{M}\right), B\left(W_{\|}, e_{j}^{M}\right)\right\rangle .
$$

Substituting (4.101) and (4.102) gives

$$
R_{F}\left(W_{\|}, e_{i}^{M}, e_{j}^{M}, W_{\|}\right)=\left\langle\left\langle\mathbf{T}, W_{\|}\right\rangle^{2} \frac{\mathbf{T}^{\prime}}{\left|F^{\prime}\right|}, \frac{\mathbf{T}^{\prime}}{\left|F^{\prime}\right|}\right\rangle-\left\langle\left\langle\mathbf{T}, W_{\|}\right\rangle \frac{\mathbf{T}^{\prime}}{\left|F^{\prime}\right|},\left\langle\mathbf{T}, W_{\|}\right\rangle \frac{\mathbf{T}^{\prime}}{\left|F^{\prime}\right|}\right\rangle=0 .
$$

Therefore (4.138) can be written using (4.100) as

$$
\delta^{2} E\left(W_{\|}, W_{\|}\right)=k \int_{S^{1}}\left\langle\mathbf{T}, \frac{W_{\|}^{\prime}}{\left|F^{\prime}\right|}\right\rangle^{2} d u
$$

We next compute $\delta^{2} E\left(W_{\|}, W_{\perp}\right)$, computing the three terms occurring in (4.139). By (4.100) and (4.101),

$$
\left\langle B\left(e_{i}^{M}, W_{\|}\right), d W_{\perp}\left(e_{j}^{M}\right)\right\rangle=\left\langle\left\langle\mathbf{T}, W_{\|}\right\rangle \frac{\mathbf{T}^{\prime}}{\left|F^{\prime}\right|}, \frac{W_{\perp}^{\prime}}{\left|F^{\prime}\right|}\right\rangle .
$$

By (4.102),

$$
-\left\langle d W_{\perp}\left(W_{\|}\right), B\left(e_{i}^{M}, e_{j}^{M}\right)\right\rangle=-\left\langle\left\langle\mathbf{T}, W_{\|}\right\rangle \frac{W_{\perp}^{\prime}}{\left|F^{\prime}\right|}, \frac{\mathbf{T}^{\prime}}{\left|F^{\prime}\right|}\right\rangle .
$$

By Example 2.2, the shape operator $S_{W_{\perp}}$ is a scalar multiplication by $\left\langle\mathbf{T}^{\prime} /\left|F^{\prime}\right|, W_{\perp}\right\rangle$. Using this, together with the first two identities in (4.100), gives

$$
-\left\langle d W_{\|}\left(e_{i}^{M}\right), S_{W_{\perp}} e_{j}^{M}\right\rangle=-\left\langle\frac{W_{\|}^{\prime}}{\left|F^{\prime}\right|},\left\langle\frac{\mathbf{T}^{\prime}}{\left|F^{\prime}\right|}, W_{\perp}\right\rangle \mathbf{T}\right\rangle
$$

We add (4.88), (4.107), and (4.108) to write a formula for $\delta^{2} E\left(W_{\|}, W_{\perp}\right)$. After simplifying, we get

$$
\delta^{2} E\left(W_{\|}, W_{\perp}\right)=-k \int_{S^{1}}\left\langle\mathbf{T}, \frac{W_{\|}^{\prime}}{\left|F^{\prime}\right|}\right\rangle\left\langle\frac{\mathbf{T}^{\prime}}{\left|F^{\prime}\right|}, W_{\perp}\right\rangle d u
$$

Finally we compute $\delta^{2} E\left(W_{\perp}, W_{\perp}\right)$. We have

$$
\left\langle S_{W_{\perp}} e_{i}^{M}, S_{W_{\perp}} e_{j}^{M}\right\rangle=\left\langle\left\langle\frac{\mathbf{T}^{\prime}}{\left|F^{\prime}\right|}, W_{\perp}\right\rangle \mathbf{T},\left\langle\frac{\mathbf{T}^{\prime}}{\left|F^{\prime}\right|}, W_{\perp}\right\rangle \mathbf{T}\right\rangle=\left\langle\frac{\mathbf{T}^{\prime}}{\left|F^{\prime}\right|}, W_{\perp}\right\rangle^{2}
$$

Substituting this into (4.140) gives

$$
\delta^{2} E\left(W_{\perp}, W_{\perp}\right)=\int_{S^{1}} P_{F}\left\langle W_{\perp}, W_{\perp}\right\rangle\left|F^{\prime}\right| d u+\int_{S^{1}}\left\{k\left\langle\frac{\mathbf{T}^{\prime}}{\left|F^{\prime}\right|}, W_{\perp}\right\rangle^{2}+q_{F}\left(\nabla^{\perp} W_{\perp}, \nabla^{\perp} W_{\perp}\right)\right\} d u .
$$


Now we add (4.105) twice (4.109) and (4.111) to obtain $\delta^{2} E(W, W)$. After simplifying, we get (4.87).

Proof of Theorem 4.10. We compute $\delta^{2} E(V, V)=\delta^{2} E\left(V_{\|}, V_{\|}\right)+2 \delta^{2} E\left(V_{\|}, V_{\perp}\right)+\delta^{2} E\left(V_{\perp}\right.$, $\left.V_{\perp}\right)$ and show that $\delta^{2} E\left(V_{\|}, V_{\|}\right), \delta^{2} E\left(V_{\perp}, V_{\perp}\right) \geq 0$ and $\delta^{2} E\left(V_{\|}, V_{\perp}\right)=0$. This will give the result. To compute each of these terms, we apply Lemma 4.21.

We begin by showing that $\delta^{2} E\left(V_{\|}, V_{\|}\right) \geq 0$. By (4.138), we have that $\delta^{2} E\left(V_{\|}, V_{\|}\right)$is the second variation formula of the harmonic functional [21, page 155]. By Theorem 4.2, we have that $F: S^{2} \rightarrow S^{2}$ is a harmonic diffeomorphism. Since a harmonic diffeomorphism between Riemannian surfaces is minimizing [8, Theorem 3], we have that the second variation $\delta^{2} E\left(V_{\|}, V_{\|}\right)$is nonnegative.

Next we prove that $\delta^{2} E\left(V_{\|}, V_{\perp}\right)=0$. Let $\hat{\mathbf{n}} \in C^{\infty}\left(S^{2}, \mathbb{R}^{n}\right)$ be the outer unit normal vector field along $S^{2}$. Write $V_{\perp}=f \hat{\mathbf{n}}$, for $f \in C^{\infty}\left(S^{2}\right)$. We compute separately the three terms occurring in (4.139) starting with

$$
\delta^{i j}\left\langle B\left(e_{i}^{M}, V_{\|}\right), d V_{\perp}\left(e_{j}^{M}\right)\right\rangle=\delta^{i j}\left\langle B\left(e_{i}^{M}, V_{\|}\right), e_{j}^{M}(f) \hat{\mathbf{n}}+f d \hat{\mathbf{n}}\left(e_{j}^{M}\right)\right\rangle .
$$

Since $\left\langle\hat{\mathbf{n}}, d \hat{\mathbf{n}}\left(e_{j}^{M}\right)\right\rangle=0$, by (2.5), we get

$$
\delta^{i j}\left\langle B\left(e_{i}^{M}, V_{\|}\right), d V_{\perp}\left(e_{j}^{M}\right)\right\rangle=\delta^{i j}\left\langle e_{j}^{M}(f) S e_{i}^{M}, V_{\|}\right\rangle=\left\langle\operatorname{Sgrad}_{g} f, V_{\|}\right\rangle,
$$

where $\operatorname{grad}_{g} f=\delta^{i j} e_{i}^{M}(f) e_{j}^{M}$ denotes the gradient of $f$ with respect to the metric $g$. Note that in this case, the shape operator $S: T_{x} S^{2} \rightarrow T_{x} S^{2}$ is a scalar multiplication by -1 . Since $g=h$, we have

$$
\delta^{i j}\left\langle B\left(e_{i}^{M}, V_{\|}\right), d V_{\perp}\left(e_{j}^{M}\right)\right\rangle=-\left\langle\operatorname{grad}_{h} f, V_{\|}\right\rangle=-V_{\|}(f) .
$$

Next we compute

$$
-\delta^{i j}\left\langle d V_{\perp}\left(V_{\|}\right), B\left(e_{i}^{M}, e_{j}^{M}\right)\right\rangle=-\delta^{i j}\left\langle V_{\|}(f) \hat{\mathbf{n}}+f d \hat{\mathbf{n}}\left(V_{\|}\right), B\left(e_{i}^{M}, e_{j}^{M}\right)\right\rangle .
$$

Proceeding as before,

$$
-\delta^{i j}\left\langle d V_{\perp}\left(V_{\|}\right), B\left(e_{i}^{M}, e_{j}^{M}\right)\right\rangle=-\delta^{i j}\left\langle V_{\|}(f) S e_{i}^{M}, e_{j}^{M}\right\rangle=V_{\|}(f) \delta^{i j}\left\langle e_{i}^{M}, e_{j}^{M}\right\rangle .
$$

Since $g=h$,

$$
-\delta^{i j}\left\langle d V_{\perp}\left(V_{\|}\right), B\left(e_{i}^{M}, e_{j}^{M}\right)\right\rangle=V_{\|}(f) 2
$$

Finally we compute

$$
-\delta^{i j}\left\langle d V_{\|}\left(e_{i}^{M}\right), S_{V_{\perp}} e_{j}^{M}\right\rangle=-\delta^{i j}\left\langle d V_{\|}\left(e_{i}^{M}\right),-f e_{j}^{M}\right\rangle .
$$

Since $g=h$,

$$
-\delta^{i j}\left\langle d V_{\|}\left(e_{i}^{M}\right), S_{V_{\perp}} e_{j}^{M}\right\rangle=f \operatorname{div} V_{\|} .
$$


Adding (4.114), (4.117), and (4.119) gives

$$
\delta^{2} E\left(V_{\|}, V_{\perp}\right)=k \int_{S^{2}} \operatorname{div}\left(f V_{\|}\right) d v o l_{g}
$$

which vanishes by the divergence theorem.

We finally prove that $\delta^{2} E\left(V_{\perp}, V_{\perp}\right) \geq 0$. For $X \in T_{x} S^{2}$, we have

$$
\nabla^{\perp} V_{\perp}(X)=\nabla_{X}^{\perp} f \hat{\mathbf{n}}=X(f) \hat{\mathbf{n}} .
$$

Hence $\nabla^{\perp} V_{\perp}=\hat{\mathbf{n}} \otimes d f$. Since $q_{F}$ is positive definite, we have $q_{F}\left(\nabla^{\perp} V_{\perp}, \nabla^{\perp} V_{\perp}\right)=q_{F}(\hat{\mathbf{n}} \otimes$ $d f, \hat{\mathbf{n}} \otimes d f) \geq 0$. Therefore, by (4.140), we have $\delta^{2} E\left(V_{\perp}, V_{\perp}\right) \geq 0$. This shows Theorem 4.10 .

We conclude this section with some technical lemmas used in the proofs of Theorems 4.9 and 4.10 .

LeMma 4.18. The map $P: \mathscr{E}_{\Omega} \times C(\Omega) \rightarrow C(M)$ given by

$$
P(F, p)=\int_{\Omega_{F}} p(F+v) \operatorname{det}\left(I-S_{v}\right) d v \frac{\sqrt{h}}{\sqrt{g}}
$$

is continuous. Here $C(M)$ and $C(\Omega)$ are considered as Banach spaces with the norm of the supreme, and also $\mathscr{E}_{\Omega}$ is considered as a subset of $C^{2}\left(M, \mathbb{R}^{n}\right)$.

Proof. Let $\epsilon>0$. We write $P(F, p)$ in terms of a continuous function defined on $J_{\Omega}^{2}$. Let $s_{p}: J_{\Omega}^{2} \rightarrow \mathbb{R}$ be given by

$$
s_{p}\left(j_{x}^{2} F\right)=\int_{\Omega_{F(x)}} p(F(x)+v) \operatorname{det}\left(I-S_{v}\left(j_{x}^{2} F\right)\right) d v \frac{\sqrt{h\left(j_{x}^{2} F\right)}}{\sqrt{g(x)}} .
$$

Then we write $(P(F, p))(x)=s_{p}\left(j_{x}^{2} F\right)$. Assume there exists a neighborhood $N \subset J_{\Omega}^{2}$ of the set $\left\{j_{x}^{2} F \in J_{\Omega}^{2}: x \in M\right\}$ such that its closure $\bar{N}$ is compact. Since $s_{p}$ is uniformly continuous on $\bar{N}$, there exists $\delta>0$ such that for all $\tilde{F} \in \mathscr{E}_{\Omega}$ with $\|F-\tilde{F}\|_{C^{2}}<\delta$, we have

$$
\sup _{M}\left|s_{p}\left(j_{x}^{2} F\right)-s_{p}\left(j_{x}^{2} \tilde{F}\right)\right|<\frac{\epsilon}{2} .
$$

Consider now the continuous function $s: J_{\Omega}^{2} \rightarrow \mathbb{R}$ given by

$$
s\left(j_{x}^{2} F\right)=\int_{\Omega_{F(x)}} \operatorname{det}\left(I-S_{v}\left(j_{x}^{2} F\right)\right) d v \frac{\sqrt{h\left(j_{x}^{2} F\right)}}{\sqrt{g(x)}} .
$$

We see that $s_{p} \leq s\|p\|_{C(\Omega)}$. Since $P$ is linear in $p$, we estimate $|P(F, p)-P(\tilde{F}, \tilde{p})|$ as

$$
\begin{aligned}
|P(F, p)-P(\tilde{F}, \tilde{p})| & \leq|P(F, p)-P(\tilde{F}, p)|+P(\tilde{F}, p-\tilde{p}) \\
& \leq \sup _{M}\left|s_{p}\left(j_{x}^{2} F\right)-s_{p}\left(j_{x}^{2} \tilde{F}\right)\right|+\sup _{M} s\left(j_{x}^{2} F\right) \cdot\|p-\tilde{p}\|_{C(\Omega)} .
\end{aligned}
$$


If $\|F-\tilde{F}\|_{C^{2}}<\delta$ and $\|p-\tilde{p}\|_{C(\Omega)}<\epsilon /\left(2 \sup _{\bar{N}} s\right)$, by $(4.124)$, we have $|P(F, p)-P(\tilde{F}, \tilde{p})|<\epsilon$. This proves the lemma.

Lemma 4.19. Let $F \in \mathscr{E}_{\Omega}$ such that $q_{F}$ is positive definite. There exists $C>0$ such that all $x \in M$ and all $(X, \xi) \in \mathbb{R}^{n} \times T_{x}^{*} M, X \neq 0, \xi \neq 0$, satisfy $q_{F}(X \otimes \xi, X \otimes \xi)>C g^{*}(\xi, \xi)|X|^{2}$.

Proof. Suppose that the lemma is false. Then, for all $n \in \mathbb{N}$, there exist $x_{n} \in M$ and $\left(X_{n}, \xi_{n}\right)$ $\in \mathbb{R}^{n} \times T_{x_{n}}^{*} M$ with $\left|X_{n}\right|^{2}=1, g^{*}(\xi, \xi)=1$, such that $q_{F}\left(X_{n} \otimes \xi_{n}, X_{n} \otimes \xi_{n}\right) \leq 1 / n$. Since $M$ is compact, there exist sequences $\left\{x_{n}\right\} \subset M,\left\{\left(X_{n}, \xi_{n}\right)\right\} \subset \mathbb{R}^{n} \times T_{x_{n}}^{*} M$ converging, respectively, to $x \in M,(X, \xi) \in \mathbb{R}^{n} \times T_{x}^{*} M$ with $|X|^{2}=1, g^{*}(\xi, \xi)=1$. Hence

$$
q_{F}(X \otimes \xi, X \otimes \xi)=\lim _{n \rightarrow \infty} q_{F}\left(X_{n} \otimes \xi_{n}, X_{n} \otimes \xi_{n}\right) \leq \lim _{n \rightarrow \infty} \frac{1}{n}=0
$$

This is a contradiction since we assumed that $q_{F}$ was positive definite. This proves the lemma.

Lemma 4.20. Let $(F, p, k) \in \mathscr{E}_{\Omega} \times C(\Omega) \times \mathbb{R}$ such that $q_{F}$ is positive definite. Then there exists a neighborhood $U \subset C^{2}\left(M, \mathbb{R}^{n}\right) \times C(\Omega) \times \mathbb{R}$ of $(F, p, k)$ such that all $(\tilde{F}, \tilde{p}, \tilde{k}) \in U$ with $\tilde{F} \in \mathscr{E}_{\Omega}$ satisfy that $q_{\tilde{F}}$ is positive definite, and the constant $C$ given by Lemma 4.19 is independent of $(\tilde{F}, \tilde{p}, \tilde{k}) \in U$.

Proof. We write $q_{F}$ in terms of a continuous function defined on $J_{\Omega}^{2}$. Let $s_{p}: J_{\Omega}^{2} \times T^{*} M \times$ $\mathbb{R}^{n} \rightarrow \mathbb{R}$ be given by

$$
\begin{aligned}
s_{p}\left(j_{x}^{2} F, \xi, X\right)=\int_{\Omega_{F(x)}} & h^{*}\left(j_{x}^{2} F\right)\left(\xi,\left[\left(I-S_{v}\left(j_{x}^{2} F\right)\right)^{-1}\right]^{*} \xi\right)\langle X, v\rangle^{2} \\
& \times p(F(x)+v) \operatorname{det}\left(I-S_{v}\left(j_{x}^{2} F\right)\right) d v \frac{\sqrt{h\left(j_{x}^{2} F\right)}}{\sqrt{g(x)}} .
\end{aligned}
$$

By formula (4.62), we can write $q_{F}(X \otimes \xi, X \otimes \xi)$ as

$$
q_{F}(X \otimes \xi, X \otimes \xi)=k g^{*}(\xi, \xi)|X|^{2}-s_{p}\left(j^{2} F, \xi, X\right)
$$

To prove the lemma we show that this formula defines a continuous function in both variables $F$ and $p$.

Consider the space $\mathscr{C}$ of continuous functions $f: J_{\Omega}^{2} \times T^{*} M \times \mathbb{R}^{n} \rightarrow \mathbb{R}$ with the topology of the uniform convergence on compact sets. Consider also the composition map $C:\left(C^{2}\left(M, \mathbb{R}^{n}\right) \cap \mathscr{E}_{\Omega}\right) \times C(\Omega) \rightarrow \mathscr{b}$ given by

$$
(C(F, p))\left(j_{x}^{2} F, \xi, X\right)=s_{p}\left(j_{x}^{2} F, \xi, X\right)
$$

We will prove that $C$ is continuous. Assume this for now and suppose that the lemma is false. Then there would exist sequences $\left\{\left(F_{n}, p_{n}, k_{n}\right)\right\} \subset\left(C^{2}\left(M, \mathbb{R}^{n}\right) \cap \mathscr{E} \Omega\right) \times C(\Omega) \times$ $\mathbb{R},\left\{\left(x_{n}, \xi_{n}\right)\right\} \subset T^{*} M$ with $g^{*}\left(\xi_{n}, \xi_{n}\right)=1$ and $\left\{X_{n}\right\} \subset S^{n-1}$, such that $\left\{\left(F_{n}, p_{n}, k_{n}\right)\right\}$ converges to $(F, p, k)$ and $q_{F_{n}}\left(X_{n} \otimes \xi_{n}, X_{n} \otimes \xi_{n}\right) \leq c g^{*}\left(\xi_{n}, \xi_{n}\right)\left|X_{n}\right|^{2}$, with $c>0$ defined as in 
Lemma 4.19. Since $M$ is compact, there exist $(x, \xi) \in T^{*} M$ with $g^{*}(\xi, \xi)=1$ and $X \in S^{n-1}$ such that a subsequence of $\left\{\left(x_{n}, \xi_{n}\right)\right\}$ converges to $(x, \xi)$ and a subsequence of $\left\{X_{n}\right\}$ converges to $X$. On the other hand, by the continuity of the composition map $C$, the sequence $\left\{C\left(F_{n}, p_{n}\right)\right\} \subset \mathscr{C}$ converges uniformly on compact sets, and therefore it is equicontinuous on compact sets. This implies

$$
q_{F}(X \otimes \xi, X \otimes \xi)=\lim _{n \rightarrow \infty} q_{F_{n}}\left(X_{n} \otimes \xi_{n}, X_{n} \otimes \xi_{n}\right) \leq c g^{*}(\xi, \xi)|X|^{2} .
$$

By Lemma 4.19, this is a contradiction since we assumed that $q_{F}$ was positive definite. This proves Lemma 4.20 .

We now prove that the composition map $C(F, p)$ is continuous. Let $(F, p) \in\left(C^{2}(M\right.$, $\left.\left.\mathbb{R}^{n}\right) \cap \mathscr{E}_{\Omega}\right) \times C(\Omega)$ and let $K_{1} \subset J_{\Omega}^{2}, K_{2} \subset T^{*} M, K_{3} \subset \mathbb{R}^{n}$ be compact subsets such that the set $\left\{j_{x}^{2} F \in J_{\Omega}^{2}: x \in M\right\} \subset K_{1}$. Let $\epsilon>0$. Since $s_{p}$ is uniformly continuous on $K=K_{1} \times$ $K_{2} \times K_{3}$, there exists $\delta>0$ such that for all $\tilde{F} \in C^{2}\left(M, \mathbb{R}^{n}\right) \cap \mathscr{E}_{\Omega}$ with $\|\tilde{F}-F\|_{C^{2}}<\delta$, we have

$$
\left|s_{p}\left(j_{x}^{2} F, \xi, X\right)-s_{p}\left(j_{x}^{2} \tilde{F}, \xi, X\right)\right|<\frac{\epsilon}{2}
$$

for all $x \in M, \xi \in K_{2}, X \in K_{3}$. Now consider the continuous function $s: J_{\Omega}^{2} \times T^{*} M \times$ $\mathbb{R}^{n} \rightarrow \mathbb{R}$ given by

$$
\begin{aligned}
& s\left(j_{x}^{2} F, \xi, X\right) \\
& \quad=\int_{\Omega_{F(x)}} h^{*}\left(j_{x}^{2} F\right)\left(\xi,\left[\left(I-S_{v}\left(j_{x}^{2} F\right)\right)^{-1}\right]^{*} \xi\right)\langle X, v\rangle^{2} \operatorname{det}\left(I-S_{v}\left(j_{x}^{2} F\right)\right) d v \frac{\sqrt{h\left(j_{x}^{2} F\right)}}{\sqrt{g(x)}} .
\end{aligned}
$$

By (4.128), we have

$$
s_{p} \leq s\|p\|_{C(\Omega)}
$$

Since $s_{p}$ is linear in $p$, then the composition map $C(F, p)$ is also linear in $p$. We estimate the supreme on $K$ of the difference $C(\tilde{F}, \tilde{p})-C(F, p)$ as

$$
\begin{aligned}
\|C(\tilde{F}, \tilde{p})-C(F, p)\|_{C(K)} & \leq\|C(\tilde{F}, \tilde{p}-p)\|_{C(K)}+\|C(\tilde{F}, p)-C(F, p)\|_{C(K)} \\
& =\sup _{K}\left|s_{\tilde{p}-p}\left(j_{x}^{2} \tilde{F}, \xi, X\right)\right|+\sup _{K}\left|s_{p}\left(j_{x}^{2} \tilde{F}, \xi, X\right)-s_{p}\left(j_{x}^{2} F, \xi, X\right)\right| .
\end{aligned}
$$

If $\|\tilde{F}-F\|_{C^{2}}<\delta$ and $\|\tilde{p}-p\|_{C(\Omega)}<\epsilon /\left(2 \sup _{K} s\right)$, then by (4.132) and (4.134), we have

$$
\|C(\tilde{F}, \tilde{p})-C(F, p)\|_{C(K)}<\sup _{K}\|\tilde{p}-p\|_{C(\Omega)}+\frac{\epsilon}{2}<\epsilon .
$$

This proves that $C$ is continuous. 
Lemma 4.21. For $F \in \mathscr{E}_{\Omega}$, let $R_{F}: T M \times T M \rightarrow \operatorname{Hom}(T M, T M)$ be the curvature tensor of $M$ with respect to the induced metric $h$. Write $V_{\|}=\pi_{F} V$ and $V_{\perp}=V-V_{\|}$. Then

$$
\delta^{2} E(V, W)=\delta^{2} E\left(V_{\|}, W_{\|}\right)+\delta^{2} E\left(V_{\|}, W_{\perp}\right)+\delta^{2} E\left(V_{\perp}, W_{\|}\right)+\delta^{2} E\left(V_{\perp}, W_{\perp}\right)
$$

where

$$
\begin{aligned}
& \delta^{2} E\left(V_{\|}, W_{\|}\right)=k \int_{M} \delta^{i j}\left\{h\left(d V_{\|}\left(e_{i}^{M}\right), d W_{\|}\left(e_{j}^{M}\right)\right)\right.\left.-h\left(R_{F}\left(V_{\|}, e_{i}^{M}\right) e_{j}^{M}, W_{\|}\right)\right\} d v o l_{g}, \\
& \delta^{2} E\left(V_{\|}, W_{\perp}\right)=k \int_{M} \delta^{i j}\left\{\left\langle B\left(e_{i}^{M}, V_{\|}\right), d W_{\perp}\left(e_{j}^{M}\right)\right\rangle-\left\langle d W_{\perp}\left(V_{\|}\right), B\left(e_{i}^{M}, e_{j}^{M}\right)\right\rangle\right. \\
&\left.\quad-\left\langle d V_{\|}\left(e_{i}^{M}\right), S_{W_{\perp}} e_{j}^{M}\right\rangle\right\} d v o l_{g}, \\
& \delta^{2} E\left(V_{\perp}, W_{\perp}\right)=\int_{M} P_{F}\left\langle V_{\perp}, W_{\perp}\right\rangle d v o l_{h} \\
&+\int_{M}\left\{k \delta^{i j}\left\langle S_{V_{\perp}} e_{i}^{M}, S_{W_{\perp}} e_{j}^{M}\right\rangle+q_{F}\left(\nabla^{\perp} V_{\perp}, \nabla^{\perp} W_{\perp}\right)\right\} d v o l_{g},
\end{aligned}
$$

where $\nabla^{\perp}$ denotes the normal connection of $F$.

Proof. We compute each of these formulas separately.

Proof of (4.138). By (3.47),

$$
\begin{aligned}
H_{F}\left(V_{\|}, W_{\|}\right)=\int_{\Omega_{F}}\left\langle\pi_{F} V_{\|}-\left\langle d V_{\|}, v\right\rangle^{\#},\left(I-S_{v}\right)^{-1}\left(\pi_{F} W_{\|}-\left\langle d W_{\|}, v\right\rangle^{\#}\right)\right\rangle \\
\cdot p(F+v) \operatorname{det}\left(I-S_{v}\right) d v,
\end{aligned}
$$

where $\pi_{F} V_{\|}=V_{\|}$. We also have $\left\langle d V_{\|}, v\right\rangle^{\#}=S_{v} V_{\|}$since, for any $Y \in T_{x} M$,

$$
\left\langle\left\langle d V_{\|}, v\right\rangle^{\#}, Y\right\rangle=\left\langle d V_{\|}(Y), v\right\rangle
$$

By (2.5),

$$
\left\langle\left\langle d V_{\|}, v\right\rangle^{\#}, Y\right\rangle=\left\langle S_{v} V_{\|}, Y\right\rangle
$$

Therefore we can write the integrand above in a contravariant way as

$$
\begin{aligned}
H_{F}\left(V_{\|}, W_{\|}\right) & =\int_{\Omega_{F}}\left\langle V_{\|}-S_{v} V_{\|},\left(I-S_{v}\right)^{-1}\left(W_{\|}-S_{v} W_{\|}\right)\right\rangle p(F+v) \operatorname{det}\left(I-S_{v}\right) d v \\
& =\int_{\Omega_{F}}\left\langle V_{\|}-S_{v} V_{\|},\left(I-S_{v}\right)^{-1}\left(I-S_{v}\right) W_{\|}\right\rangle p(F+v) \operatorname{det}\left(I-S_{v}\right) d v
\end{aligned}
$$


Simplifying and using (2.5) gives

$$
\begin{aligned}
H_{F}\left(V_{\|}, W_{\|}\right)= & \int_{\Omega_{F}}\left(\left\langle V_{\|}, W_{\|}\right\rangle-\left\langle d W_{\|}\left(V_{\|}\right), v\right\rangle\right) p(F+v) \operatorname{det}\left(I-S_{v}\right) d v \\
= & \left\langle V_{\|}, W_{\|}\right\rangle \int_{\Omega_{F}} p(F+v) \operatorname{det}\left(I-S_{v}\right) d v \\
& +\left\langle d W_{\|}\left(V_{\|}\right),-\int_{\Omega_{F}} p(F+v) \operatorname{det}\left(I-S_{v}\right) v d v\right\rangle .
\end{aligned}
$$

By the definition of $P_{F}$ and $\tau_{F}$,

$$
H_{F}\left(V_{\|}, W_{\|}\right)=\left\langle V_{\|}, W_{\|}\right\rangle P_{F}+\left\langle d W_{\|}\left(V_{\|}\right), \tau_{F}\right\rangle
$$

Using this in the definition of $Q_{F}$ gives

$$
\begin{aligned}
& Q_{F}\left(V_{\|}, W_{\|}\right) \\
& \quad=k \delta^{i j}\left\langle d V_{\|}\left(e_{i}^{M}\right), d W_{\|}\left(e_{j}^{M}\right)\right\rangle-\left(\left\langle V_{\|}, W_{\|}\right\rangle P_{F}+\left\langle d W_{\|}\left(V_{\|}\right), \tau_{F}\right\rangle\right) \frac{\sqrt{h}}{\sqrt{g}} .
\end{aligned}
$$

By (4.8),

$$
\begin{aligned}
Q_{F}\left(V_{\|}, W_{\|}\right)= & k \delta^{i j}\left\langle d V_{\|}\left(e_{i}^{M}\right), d W_{\|}\left(e_{j}^{M}\right)\right\rangle-\left\langle V_{\|}, W_{\|}\right\rangle P_{F} \frac{\sqrt{h}}{\sqrt{g}} \\
& -\left\langle d W_{\|}\left(V_{\|}\right), k \delta^{i j} B\left(e_{i}^{M}, e_{j}^{M}\right)\right\rangle .
\end{aligned}
$$

We write the first term $\left\langle d V_{\|}\left(e_{i}^{M}\right), d W_{\|}\left(e_{j}^{M}\right)\right\rangle$ separating it into normal and tangential components of $d V_{\|}\left(e_{i}^{M}\right)$ and $d W_{\|}\left(e_{j}^{M}\right)$. We get

$$
\begin{aligned}
Q_{F}\left(V_{\|}, W_{\|}\right)= & k \delta^{i j}\left\langle B\left(e_{i}^{M}, V_{\|}\right), B\left(e_{j}^{M}, W_{\|}\right)\right\rangle+k \delta^{i j} h\left(d V_{\|}\left(e_{i}^{M}\right), d W_{\|}\left(e_{j}^{M}\right)\right) \\
& -\left\langle V_{\|}, W_{\|}\right\rangle P_{F} \frac{\sqrt{h}}{\sqrt{g}}-\left\langle B\left(V_{\|}, W_{\|}\right), k \delta^{i j} B\left(e_{i}^{M}, e_{j}^{M}\right)\right\rangle,
\end{aligned}
$$

where we also used $\left(d W_{\|}\left(V_{\|}\right)\right)_{\perp}=B\left(V_{\|}, W_{\|}\right)$. By the Gauss equation [3, page 135],

$$
\begin{aligned}
& Q_{F}\left(V_{\|}, W_{\|}\right) \\
& \quad=k \delta^{i j}\left(h\left(d V_{\|}\left(e_{i}^{M}\right), d W_{\|}\left(e_{j}^{M}\right)\right)-R_{F}\left(V_{\|}, e_{i}^{M}, e_{j}^{M}, W_{\|}\right)\right)-\left\langle V_{\|}, W_{\|}\right\rangle P_{F} \frac{\sqrt{h}}{\sqrt{g}} .
\end{aligned}
$$

By (4.63),

$$
\delta^{2} E\left(V_{\|}, W_{\|}\right)=\int_{M}\left\{k \delta^{i j}\left(h\left(d V_{\|}\left(e_{i}^{M}\right), d W_{\|}\left(e_{j}^{M}\right)\right)-R_{F}\left(V_{\|}, e_{i}^{M}, e_{j}^{M}, W_{\|}\right)\right)\right\} d v o l_{g},
$$

where the term involving $P_{F}$ has been canceled. This gives (4.138). 
Proof of (4.139). By (3.47),

$$
\begin{aligned}
H_{F}\left(V_{\|}, W_{\perp}\right)=\int_{\Omega_{F}} & \left\langle\pi_{F} V_{\|}-\left\langle d V_{\|}, v\right\rangle^{\#},\left(I-S_{v}\right)^{-1}\left(\pi_{F} W_{\perp}-\left\langle d W_{\perp}, v\right\rangle^{\#}\right)\right\rangle \\
& \times p(F+v) \operatorname{det}\left(I-S_{v}\right) d v .
\end{aligned}
$$

Using the identities $\pi_{F} V_{\|}=V_{\|},\left\langle d V_{\|}, v\right\rangle^{\#}=S_{\nu} V_{\|}$, and $\pi_{F} W_{\perp}=0$ gives

$$
\begin{aligned}
\left\langle V_{\|}\right. & \left.-S_{v} V_{\|},\left(I-S_{v}\right)^{-1}\left(-\left\langle d W_{\perp}, v\right\rangle^{\#}\right)\right\rangle \\
& =\left\langle\left(I-S_{v}\right) V_{\|},\left(I-S_{v}\right)^{-1}\left(-\left\langle d W_{\perp}, v\right\rangle^{\#}\right)\right\rangle .
\end{aligned}
$$

Since $\left(I-S_{v}\right) *$ is selfadjoint,

$$
\begin{aligned}
\left\langle V_{\|}\right. & \left.-S_{v} V_{\|},\left(I-S_{v}\right)^{-1}\left(-\left\langle d W_{\perp}, v\right\rangle^{\#}\right)\right\rangle \\
& =\left\langle V_{\|},\left(-\left\langle d W_{\perp}, v\right\rangle^{\#}\right)\right\rangle=\left\langle-d W_{\perp}\left(V_{\|}\right), v\right\rangle=\left\langle v,-d W_{\perp}\left(V_{\|}\right)\right\rangle .
\end{aligned}
$$

Substituting into (4.152),

$$
\begin{aligned}
H_{F}\left(V_{\|}, W_{\perp}\right) & =\int_{\Omega_{F}}\left\langle v,-d W_{\perp}\left(V_{\|}\right)\right\rangle p(F+v) \operatorname{det}\left(I-S_{v}\right) d v \\
& =\left\langle-\int_{\Omega_{F}} p(F+v) \operatorname{det}\left(I-S_{v}\right) v d v, d W_{\perp}\left(V_{\|}\right)\right\rangle .
\end{aligned}
$$

By the definition of $\tau_{F}$,

$$
H_{F}\left(V_{\|}, W_{\perp}\right)=\left\langle\tau_{F}, d W_{\perp}\left(V_{\|}\right)\right\rangle
$$

Using this in the definition of $Q_{F}$ gives

$$
Q_{F}\left(V_{\|}, W_{\perp}\right)=k \delta^{i j}\left\langle d V_{\|}\left(e_{i}^{M}\right), d W_{\perp}\left(e_{j}^{M}\right)\right\rangle-\left\langle\tau_{F}, d W_{\perp}\left(V_{\|}\right)\right\rangle \frac{\sqrt{h}}{\sqrt{g}} .
$$

By (4.8),

$$
Q_{F}\left(V_{\|}, W_{\perp}\right)=k \delta^{i j}\left\langle d V_{\|}\left(e_{i}^{M}\right), d W_{\perp}\left(e_{j}^{M}\right)\right\rangle-k \delta^{i j}\left\langle B\left(e_{i}^{M}, e_{j}^{M}\right), d W_{\perp}\left(V_{\|}\right)\right\rangle
$$

We write the term $\left\langle d V_{\|}\left(e_{i}^{M}\right), d W_{\perp}\left(e_{j}^{M}\right)\right\rangle$ separating it into normal and tangential components of $d V_{\|}\left(e_{i}^{M}\right)$ and $d W_{\perp}\left(e_{j}^{M}\right)$. We get

$$
\begin{aligned}
Q_{F}\left(V_{\|}, W_{\perp}\right)= & k \delta^{i j}\left(-\left\langle S_{W_{\perp}} e_{j}^{M}, d V_{\|}\left(e_{i}^{M}\right)\right\rangle+\left\langle B\left(e_{i}^{M}, V_{\|}\right), d W_{\perp}\left(e_{j}^{M}\right)\right\rangle\right) \\
& -k \delta^{i j}\left\langle B\left(e_{i}^{M}, e_{j}^{M}\right), d W_{\perp}\left(V_{\|}\right)\right\rangle .
\end{aligned}
$$

Using (4.63) gives (4.140). 
Proof of (4.140). By (3.47),

$$
\begin{aligned}
H_{F}\left(V_{\perp}, W_{\perp}\right)= & \int_{\Omega_{F}}\left\langle\pi_{F} V_{\perp}-\left\langle d V_{\perp}, v\right\rangle^{\#},\left(I-S_{v}\right)^{-1}\left(\pi_{F} W_{\perp}-\left\langle d W_{\perp}, v\right\rangle^{\#}\right)\right\rangle \\
& \times p(F+v) \operatorname{det}\left(I-S_{v}\right) d v \\
= & \int_{\Omega_{F}}\left\langle\left\langle d V_{\perp}, v\right\rangle^{\#},\left(I-S_{v}\right)^{-1}\left\langle d W_{\perp}, v\right\rangle^{\#}\right\rangle p(F+v) \operatorname{det}\left(I-S_{v}\right) d v .
\end{aligned}
$$

We have $\left\langle d V_{\perp}, v\right\rangle^{\#}=\left\langle\left(d V_{\perp}\right)_{\perp}, v\right\rangle^{\#}$. Hence

$$
H_{F}\left(V_{\perp}, W_{\perp}\right)=\int_{\Omega_{F}}\left\langle\left\langle\left(d V_{\perp}\right)_{\perp}, v\right\rangle^{\#},\left(I-S_{v}\right)^{-1}\left(\left\langle\left(d W_{\perp}\right)_{\perp}, v\right\rangle^{\#}\right)\right\rangle p(F+v) \operatorname{det}\left(I-S_{v}\right) d v .
$$

Using this in the definition of $Q_{F}$ gives

$$
\begin{aligned}
Q_{F}\left(V_{\perp}, W_{\perp}\right) \\
=k \delta^{i j}\left\langle d V_{\perp}\left(e_{i}^{M}\right), d W_{\perp}\left(e_{j}^{M}\right)\right\rangle \\
\quad-\int_{\Omega_{F}}\left\langle\left\langle\left(d V_{\perp}\right)_{\perp}, v\right\rangle^{\#},\left(I-S_{v}\right)^{-1}\left(\left\langle\left(d W_{\perp}\right)_{\perp}, v\right\rangle^{\#}\right)\right\rangle p(F+v) \operatorname{det}\left(I-S_{v}\right) d v \frac{\sqrt{h}}{\sqrt{g}} .
\end{aligned}
$$

We write $\left\langle d V_{\perp}\left(e_{i}^{M}\right), d W_{\perp}\left(e_{j}^{M}\right)\right\rangle$ separating it into normal and tangential components of $d V_{\perp}\left(e_{i}^{M}\right)$ and $d W_{\perp}\left(e_{j}^{M}\right)$. By (2.9),

$$
\begin{aligned}
Q_{F}\left(V_{\perp}, W_{\perp}\right) \\
=k \delta^{i j}\left\langle S_{V_{\perp}} e_{i}^{M}, S_{W_{\perp}} e_{j}^{M}\right\rangle+k \delta^{i j}\left\langle\left(d V_{\perp}\left(e_{i}^{M}\right)\right)_{\perp},\left(d W_{\perp}\left(e_{j}^{M}\right)\right)_{\perp}\right\rangle \\
\quad-\int_{\Omega_{F}}\left\langle\left\langle\left(d V_{\perp}\right)_{\perp}, v\right\rangle^{\#},\left(I-S_{v}\right)^{-1}\left(\left\langle\left(d W_{\perp}\right)_{\perp}, v\right\rangle^{\#}\right)\right\rangle p(F+v) \operatorname{det}\left(I-S_{v}\right) d v \frac{\sqrt{h}}{\sqrt{g}} .
\end{aligned}
$$

This can be written in terms of $q_{F}$ as

$$
Q_{F}\left(V_{\perp}, W_{\perp}\right)=k \delta^{i j}\left\langle S_{V_{\perp}} e_{i}^{M}, S_{W_{\perp}} e_{j}^{M}\right\rangle+q_{F}\left(\nabla^{\perp} V_{\perp}, \nabla^{\perp} W_{\perp}\right) .
$$

Using (4.63) gives (4.140).

\section{Conclusions}

In this paper we studied the variational properties of the functional

$$
E: \mathscr{E}_{\Omega} \longrightarrow \mathbb{R}
$$


on the class of $\Omega$-regular embeddings of a manifold $M$ into a closed region $\Omega \subset \mathbb{R}^{n}$. We characterized the critical embeddings of this functional by the Euler-Lagrange equation (4.8). In the special case when $M$ is diffeomorphic to the circle, we gave conditions under which critical embeddings are local minima (Theorem 4.9).

A number of open questions remain. Two important special cases of the manifold fitting problem are the curve and surface fitting problems. The problem of global existence of regular local minima remains open, even in the relatively simple case of curves. In the case of surfaces, we have not yet succeeded in finding an easily verifiable sufficient condition for a critical surface to be a local minimum of $E$.

\section{References}

[1] J. Banfield and A. E. Raftery, Ice floe identification in satellite images using mathematical morphology and clustering about principal curves, J. Amer. Statist. Assoc. 87 (1992), no. 417, $7-16$.

[2] J. F. Brinkley, Knowledge-driven ultrasonic three-dimensional organ modeling, IEEE Trans. Pattern Anal. Machine Intell. 7 (1985), no. 4, 431-441.

[3] M. P. do Carmo, Riemannian Geometry, Mathematics: Theory \& Applications, Birkhäuser Boston, Massachusetts, 1992.

[4] T. Duchamp and W. Stuetzle, Extremal properties of principal curves in the plane, Ann. Statist. 24 (1996), no. 4, 1511-1520.

[5] L. Fang and D. C. Gossard, Reconstruction of smooth parametric surfaces from unorganized data points, Curves and Surfaces in Computer Vision and Graphics III (J. D. Warren, ed.), Proceedings of SPIE, vol. 1830, The International Society for Optical Engineering, Bellingham, 1992, pp. 226-236.

[6] A. Goshtasby, Surface reconstruction from scattered measurements, Curves and Surfaces in Computer Vision and Graphics III (J. D. Warren, ed.), Proceedings of SPIE, vol. 1830, The International Society for Optical Engineering, Bellingham, 1992, pp. 247-256.

[7] T. Hastie and W. Stuetzle, Principal curves, J. Amer. Statist. Assoc. 84 (1989), no. 406, 502-516.

[8] F. Hélein, Harmonic diffeomorphisms between Riemannian manifolds, Variational Methods (Paris, 1988), Progr. Nonlinear Differential Equations Appl., vol. 4, Birkhäuser Boston, Massachusetts, 1990, pp. 309-318.

[9] H. Hoppe, T. DeRose, T. Duchamp, J. McDonald, and W. Stuetzle, Surface reconstruction from unorganized points, Comput. Graphics 26 (1992), no. 2, 71-78.

[10] Mesh optimization, Comput. Graphics 27 (1993), 19-26.

[11] V. Khatskevich and D. Shoiykhet, Differentiable Operators and Nonlinear Equations, Operator Theory: Advances and Applications, vol. 66, Birkhäuser Verlag, Basel, 1994.

[12] T. Kludas, Three Dimensional Surface Reconstruction with the Zeiss Photogrammetric Industrial Measurement System InduSURF Digital, Automotive Automation, Croydon, UK, 1995.

[13] C. T. Lim, G. M. Turkiyyah, M. A. Ganter, and D. W. Storti, Implicit reconstruction of solids from cloud point sets, Proceeding of the 3rd ACM Symposium on Solid Modeling and Applications (Salt Lake City, Utah, 1995), ACM Press, New York, 1995, pp. 393-402.

[14] R. W. Martin, G. Bashein, M. L. Nessly, and F. H. Sheehan, Methodology for three-dimensional reconstruction of the left ventricle from transesophageal echocardiograms, Ultrasound Med. Biol. 19 (1993), no. 1, 27-38.

[15] S. Muraki, Volumetric shape description of range data using "Blobby Model", Comput. Graphics 25 (1991), no. 4, 227-235.

[16] R. B. Schudy and D. H. Ballard, Model detection of cardiac chambers in ultrasound images, Tech. Report 12, Computer Science Department, University of Rochester, New York, 1978. 
[17] - Towards an anatomical model of heart motion as seen in 4-d cardiac ultrasound data, Proceedings of the 6th Conference on Computer Applications in Radiology and ComputerAided Analysis of Radiological Images, 1979.

[18] F. H. Sheehan, M. P. Feneley, N. P. DeBruijn, J. S. Rankin, J. W. Davis, E. L. Bolson, P. S. Glass, and F. M. Clements, Quantitative analysis of regional wall thickening by transesophageal echocardiography, J. Thorac. Cardiovasc. Surg. 103 (1992), no. 2, 347-354.

[19] F. Solina and R. Bajcsy, Recovery of parametric models from range images: the case for superquadrics with global deformations, IEEE Trans. Pattern Anal. Machine Intell. 12 (1990), no. 2, 131-147.

[20] S. Sunthankar, Three-dimensional solid object laser scanner, Intelligent Robots and Computer Vision XI: Biological, Neural Net, and 3D Methods (D. P. Casasent, ed.), Proceedings of SPIE, vol. 1826, The International Society for Optical Engineering, Bellingham, 1992, pp. 495-510.

[21] H. Urakawa, Calculus of Variations and Harmonic Maps, Translations of Mathematical Monographs, vol. 132, American Mathematical Society, Rhode Island, 1993.

[22] B. C. Vemuri, A. Mitiche, and J. K. Aggarwal, Curvature-based representation of objects from range data, Image Vision Comput. 4 (1986), no. 2, 107-114.

José L. Martínez-Morales: Instituto de Matemáticas, Universidad Nacional Autónoma de México, A.P. 273, Admon. de Correos \#3, C.P. 62251 Cuernavaca, Morelos, Mexico

E-mail address: martinez@matcuer.unam.mx 


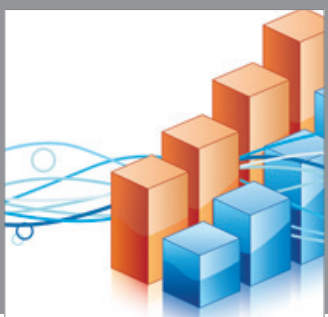

Advances in

Operations Research

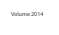

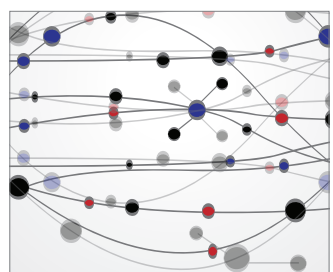

\section{The Scientific} World Journal
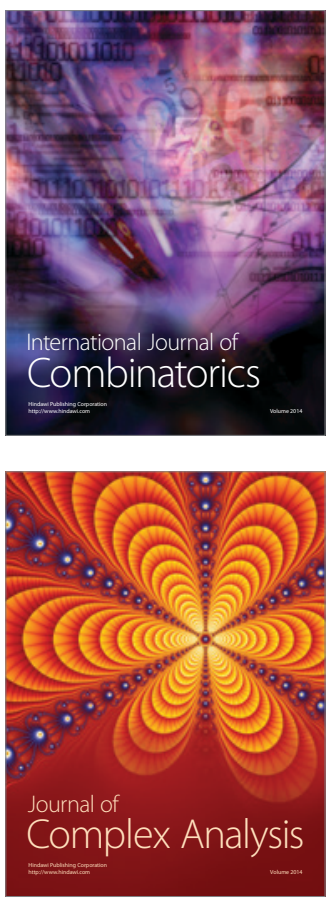

International Journal of

Mathematics and

Mathematical

Sciences
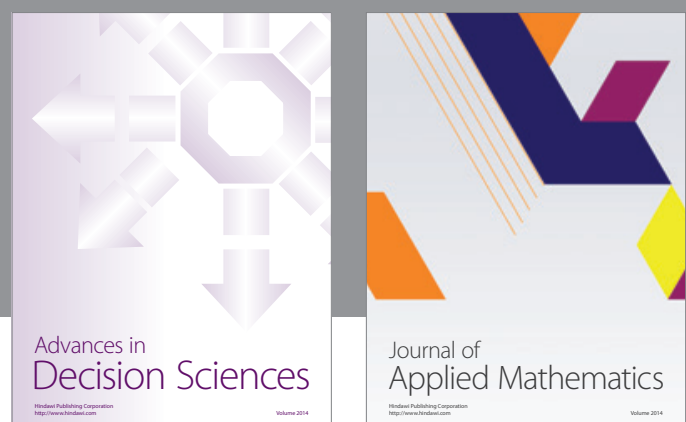

Journal of

Applied Mathematics
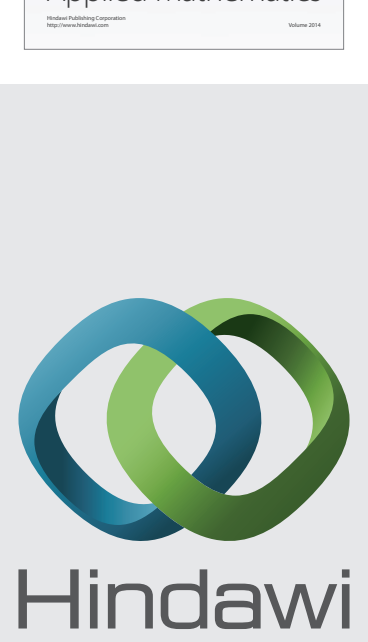

Submit your manuscripts at http://www.hindawi.com
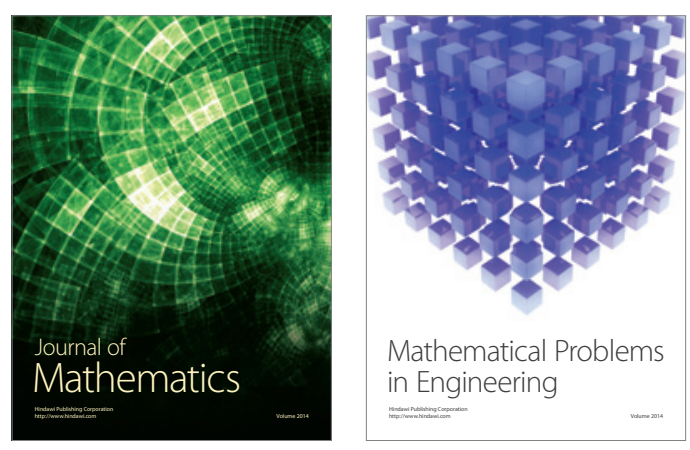

Mathematical Problems in Engineering
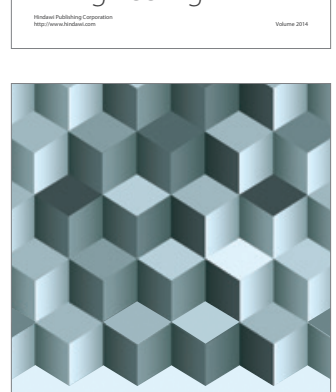

Journal of

Function Spaces
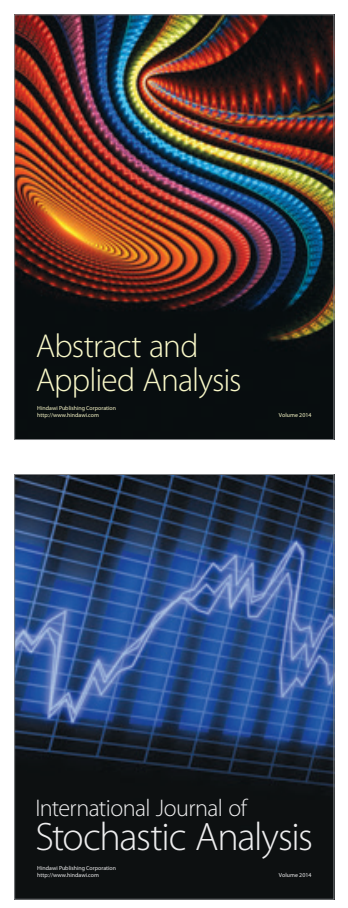

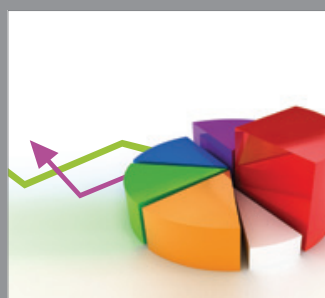

ournal of

Probability and Statistics

Promensencen
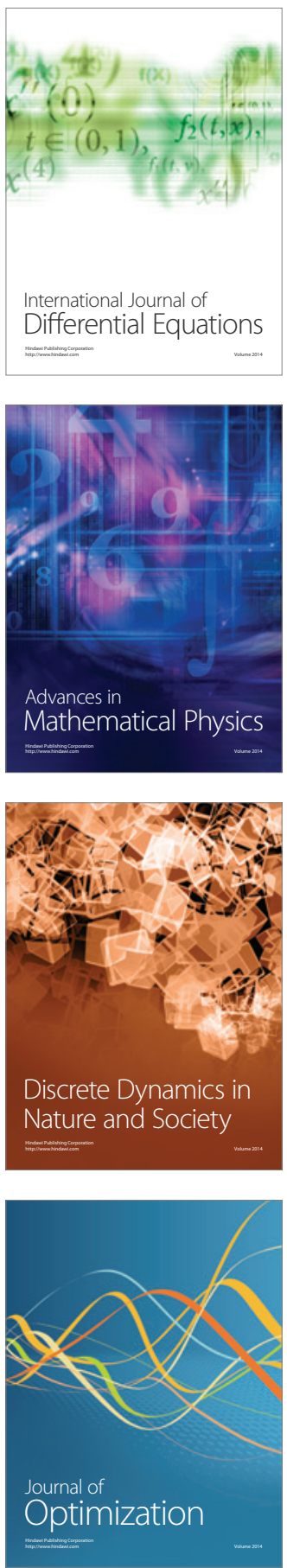